11 Oct. 2016 (submitted). Comments welcome.

\title{
The Message Shapes Phonology
}

\section{Kathleen Currie Hall Elizabeth Hume T. Florian Jaeger Andrew Wedel}

Univ. of British Columbia / Univ. of Canterbury / Univ. of Rochester / Univ. of Arizona

The fundamental problem of communication is that of reproducing at one point either exactly or approximately a message selected at another point.

Claude Shannon (1948: 379)

\begin{abstract}
${ }^{*}$
Based on a diverse and complementary set of theoretical and empirical findings, we describe an approach to phonology in which sound patterns are shaped by the trade-off between biases supporting message transmission accuracy and resource cost. We refer to this approach as Message-Oriented Phonology. The evidence suggests that these biases influence the form of messages, defined with reference to a language's morphemes, words or higher levels of meaning, rather than influencing phonological categories directly. Integrating concepts from information theory and Bayesian inference with the existing body of phonological research, we propose a testable model of phonology that makes quantitative predictions. Moreover, we show that approaching language as a system of message transfer provides greater explanatory coverage of a diverse range of sound patterns.
\end{abstract}

* Acknowledgements.

Approx. 22k words plus references. 


\section{Introduction}

A central goal of phonology is the development of a causally explanatory model of sound patterns that accounts for both cross-linguistic commonalities and language-specific details. One insight that has emerged from research on this question is that the development of phonological systems over time seems to be influenced in part by factors that are known to operate during the perception or production of language (for a recent review, see Yu 2013, inter alia). For example, a number of properties of phonological systems are compatible with the hypothesis that perceptual biases and constraints can shape phonology (e.g. Boersma 1998, 2011; Flemming 1995; Hume 2004; Hume \& Johnson 2001; Jun 1995; Kirchner, Hayes \& Steriade 2004; Padgett 1995; Silverman 1997; Steriade 2001). Similarly, there seem to be influences of articulatory biases and constraints (e.g. Archangeli \& Pulleyblank 1994; Browman \& Goldstein 1986; Kirchner 2001; Kochetov 2002). Another line of research has advanced our understanding of how phonological systems function and evolve in communities over time, including the accumulated effects of articulatory and perceptual factors (e.g., Ohala 1981; Lindblom 1990; Kingston 2007; Kirby 2010; Kohler 1990; Bybee 2001; Blevins 2004; Pierrehumbert 2001a; Sóskuthy 2015; Wedel 2006; Hay \& Maclagan 2012).

Here, we aim to contribute to this larger project by revisiting and expanding on the idea that phonology as a system subserves the reliable transmission of meaning-bearing units, i.e. messages, and that this is ultimately the reason why factors such as perceptual prominence come to play a role in shaping phonological systems. In one form or another, this idea has driven research in phonology since at least Schuchardt (1872) and Zipf (1932); see, e.g., Jakobson, Cherry \& Halle 1952; Martinet 1972; Lindblom 1990; Silverman 1997; Boersma 1998, 2009, 2011; Bybee 2001; and Kirby 2010. Two more recent developments prompt us to revisit this idea.

First, probability theory, information theory (Shannon 1948), and Bayesian inference (Bayes 1763; Laplace 1886) — all influential in the early days of the cognitive sciences - have re-entered linguistic theory building. These mathematical theories allow us to formalize and quantify what it means for a linguistic system to be "effective" for message transmission, for example, by advancing our understanding of the role of redundancy (see, e.g., Genzel \& Charniak 2002; Jurafsky 2003; Ferrer i Cancho 2005; Ferrer i Cancho \& Díaz-Guilera 2007; Jaeger 2006; Levy \& Jaeger 2007; Piantadosi et al. 2012; Pate \& Goldwater 2015). These approaches have been 
exploited, for example, to understand speakers' preferences during online language production and whether they facilitate message transmission - from phonetics (e.g., Aylett \& Turk 2004; Nielsen \& Wilson 2008; Pluymaekers et al. 2005a; van Son \& Pols 2003; Torreira \& Ernestus, 2009; Wilson \& Davidson 2013) and phonological encoding (e.g., Bell et al. 2003; Gahl \& Garnsey 2004; Raymond et al. 2006; Tily \& Kuperman 2012; Cohen Priva 2015) to lexical and grammatical encoding (e.g., Jaeger 2010; Kravtchenko 2014; Kurumada \& Jaeger 2015; Tily \& Piantadosi 2009; for review of these literatures, see Jaeger \& Buz in press). The same broad class of mathematical frameworks has been influential in modeling language comprehension-from speech perception (e.g., Clayards et al 2008; Feldman et al. 2009; Kleinschmidt \& Jaeger 2015, 2016), to morphological processing (e.g., del Prado Martín, Kostić, \& Baayen 2004), word recognition (e.g., Norris 2006; Norris \& McQueen 2008), sentence processing (e.g., Gibson et al. 2013; Hale 2005; Jurafsky 1996; Jurafsky \& Narayanan 2002; Levy 2008), and beyond (e.g., Frank \& Goodman 2012; Franke 2009; Franke \& Jäger 2015; Kehler \& Rohde 2013; Potts, Lassiter, Levy, \& Frank 2015; Rohde, Levy, \& Kehler 2011; for review of many of these literatures, see also Kuperberg \& Jaeger 2016).

Second, research has advanced our empirical understanding of what makes some parts of the speech signal a priori perceptually more or less supportive of message transmission (e.g., Cutler et al. 1985; Marslen-Wilson \& Zwitserlood 1989). Building on this research, for example, certain positions in syllables or words have been identified as more prominent (e.g., Beckman 1997; Smith 2001; Steriade 2001; Zoll 2003; Barnes 2006), a property that, we argue, turns out to be critical in understanding how pressures on message transmission come to shape phonology.

Taken together, we argue, these two advances provide the critical ingredients that allow us to take the next step in developing a predictive theoretical framework of how communicative goals, and thus message transmission, shape phonology. Indeed, information theoretic and Bayesian frameworks have been applied to the study of lexical (e.g., Mahowald et al. 2013; Manin 2006; Piantadosi et al. 2011), morphological (e.g., Ackerman, Blevins, \& Malouf 2009; Blevins 2013; Sims \& Parker 2016), and grammatical phenomena (e.g., Gibson et al. 2013b; Gildea \& Jaeger submitted; Maurits, Navarro \& Perfors 2010), including questions about why human languages developed grammatical structure to begin with (e.g., Nowak et al. 2000; Plotkin \& Nowak 2000). Of particular relevance to the current purpose, case studies have demonstrated that some properties of phonological systems can be derived from information theoretic or Bayesian 
considerations about message transmission, including work on allophony (Hall 2009, 2012), assimilation (Turnbull, Seyfarth, Hume \& Jaeger, in prep), epenthesis (Hume \& Bromberg 2005; Hong 2011; Hume et al. 2013; Tily \& Kuperman 2012), markedness (Hume 2005; Hume et al. 2016), phoneme mergers (Wedel et al. 2013a,b), vowel harmony (Goldsmith \& Riggle 2012), and consonant deletion (Cohen Priva 2015). What has been lacking to date is a larger picture of how these individual pieces fit together to predict the shape of phonological systems more generally (though see, e.g., Cohen Priva 2012; Flemming 2010; Wedel 2012, Hall 2013; Hume \& Mailhot 2013; Moulin-Frier et al. 2015 for steps in this direction). Providing this larger picture is our goal here.

Central to the current approach is the view that information transmission in language can be modeled as Bayesian (and thus probabilistic) inference processes. Linguistic meaning is conveyed through the encoding of a message by the speaker into a continuous phonetic signal. Processing of this signal by the listener into a message can be conceptually broken down into multiple levels. ${ }^{1}$ For example, the continuous phonetic signal can be mapped onto higher-level categories such as phonological units in some context (abbreviated PUCs; these are any elements that can be referenced by a phonological grammar, such as features, segments or syllables). Likewise, the phonetic signal and PUCs themselves can be mapped onto higher, meaning-bearing units such as morphemes and words, which we term messages. Information transmission at all of these levels can be modeled as a Bayesian inference process. One can, for example, calculate both how likely the accurate transmission of a particular PUC is, given properties of the phonetic signal, and how likely the accurate transmission of a particular meaning-bearing unit is, given the evidence for a set of PUCs.

\footnotetext{
${ }^{1}$ It is possible to think of speech perception as a chain of transmission processes, compressing acoustic signal into increasingly higher-level (more abstract) linguistic categories. These chains could correspond to the more or less clearly delineated 'stages' or 'levels of representation' often referred to in psycholinguistic or linguistic theories, including the recognition of phonological features, phonemes, words, etc. The chains could, however, also differ for different parts of the signal, for example, because of frequent co-occurrence of that signal part with a certain categorization (e.g., some parts of the signal might directly be matched to words; cf. chunk-based accounts, Arnon \& Snider 2010; Christiansen \& Chater 2016; or certain hierarchical predictive frameworks, e.g., Farmer et al. 2013, O'Donnell 2015; Kuperberg \& Jaeger 2016). Here we refrain from committing to specific levels of representation, partly because the neuroscientific evidence for some of them remains lacking: for instance, while cortical recording has provided relatively direct evidence that phonological features are neurologically coded (Chang et al. 2010; Pasley et al. 2012; Bouchard et al. 2013; Mesgarani et al. 2014), the same studies have found little evidence that there are cells or groups of cells that code phonemes. For related discussion and references, see also Norris \& McQueen (2008, p. 362).
} 
Viewed this way, phonology exists at an intermediary level in this set of inference processes from acoustic signal to message. This, we will argue, has far-reaching consequences. Traditionally, phonological research has been 'sound-centric', where PUCs are the object of variability and change. From a sound-centric perspective, the focus is on the realization and recognition of PUCs (given, e.g., the quality of phonetic cues to a particular PUC, Steriade 2008). As we discuss in Section 4.4, focusing solely on PUCs as targets makes predictions that are incompatible with well-known phonological patterns. Only by also taking into account the goal of transmitting meaning-bearing units ("messages"), we argue, can one correctly predict the broad range of observed sound patterns. For this reason, we refer to the proposed approach as Message-Oriented Phonology (MOP). Indeed, as we show, MOP predicts attested typologically common patterns $(\S 4)$ as well as observed language-specific patterns (§4.5). MOP further constrains how language-specific properties interact with general biases (e.g., from production and perception). To foreshadow our argument, MOP predicts that within a given language, context-specific differences in the amount of information PUCs carry about meaning-bearing messages influence the extent to which general articulatory and perceptional biases affect the realization of PUCs. This, as we discuss in more length below, holds the promise of addressing the long-standing question of how such language-specific differences can be accommodated in accounts that ground phonology in general articulatory or perceptual constraints (e.g., Archangeli \& Pulleyblank 1994, Steriade 2008). Another immediate consequence of this view is that a full understanding of phonological structure in any language requires reference to context beyond the local phonetic and (morpho-) phonological environment.

One objection to approaches like the one pursued here is that they have more degrees of freedom, in contrast to approaches that refer solely to proposed universal properties of sound systems, which often make easily falsifiable predictions. Our goal here is to show that it is possible to make progress in addressing this challenge by grounding our approach in the mathematical theories mentioned above. In this way, MOP goes beyond many related proposals formulated in the usage-based tradition. For example, while the probabilistic quantities that are assumed to predict usage effects are often left unspecified in previous work or purely chosen based on convenience (e.g., frequency vs. conditional probability), the theorems MOP is built on remove many of these degrees of freedom. The result, we argue, is a theory that is parsimonious, because it is rooted in abstract principles of communication systems, and general, because it 
applies these principles to a wide range of phenomena. Further, it is testable because it allows researchers to derive quantitative predictions.

We will use the proposed MOP framework as a foundation for reasoning about where in a given phonetic signal it would be most beneficial to invest or divest resources to maximize the likelihood of accurate, yet cost-effective message transmission (i.e. effective communication, a notion we elaborate on in §3.1). As we will review, a growing body of evidence indicates that variation in the realization of PUCs is in fact biased toward phonetic enhancement of PUCs that contribute more to transmission of lexemes and other meaning-bearing units, and toward phonetic reduction of PUCs that contribute less. MOP then predicts that a cognitive bias toward effective message transmission will interact with more general perceptual and articulatory biases to influence the prototypical form of PUCs in a given speech community, and thereby shape that phonological system over time (see, e.g., Blevins \& Wedel 2009; Cohen Priva 2008; Wedel 2012; Zipf 1949). Indeed, as we will review, both traditional linguistic and laboratory evidence supports the idea that phonological patterns arise through biases on the phonetic form of meaning-based units, rather than on phonological units themselves, lending support to the ideas we seek to advance here.

This idea was arguably realized early with regard to the role of word-level representations in phonological processes (for discussion and references, see Schuchardt 1872, p. 57-59). Today word-level representations play a critical role in exemplar-based approaches to sound change (e.g., Bybee 2002b; Ernestus 2011; Pierrehumbert 2001, 2002; Wedel 2006). There are, however, also important differences between these and the present proposal. Work in the exemplar-based and related traditions has focused on the "practice" effects of repeated usage of words, which allow biases on production and perception to gradually shift the character of representations. MOP focuses on biases toward accurate and cost-effective transmission of meaning-bearing units (including words). This leads us to anchor MOP in mathematical considerations about inference and communication (Sections 3 and 4). This anchoring has consequences for precisely what usage-related measures MOP predicts to affect phonology (Sections 3 and 6). Finally, it makes explicit considerations that have (largely implicitly) guided theoretical reasoning about the production-perception loop in exemplar-based accounts (Pierrehumbert 2001; Wedel 2006).

To introduce the MOP approach we will concentrate on a diverse group of asymmetrical phonological patterns that can be framed, in general terms, as being weak or strong, exemplified 
by lenitions, fortitions, and other forms of reduction and enhancement. As we describe in Section 2 just below, a general puzzle is presented by the diverse domains that display apparently similar strong and weak phonological pattern types. In Section 3, we provide background on language as a system of message transmission, and review evidence that factors influencing message transmission do in fact shape language variation and change. In Section 4 we return to the puzzle described in Section 2 and provide an explanatory and predictive model of phonology that is able to unify strong and weak phonological patterns through their common role in creating an effective message transmission system. In Section 5, we discuss how the proposed reasoning might be extended to other phonological patterns, exploring assimilation, epenthesis, boundary phenomena, and markedness more broadly. Finally, in Section 6 we discuss the connections between this approach and previous work, as noted above. Section 7 summarizes the main findings and provides suggestions for further research.

\section{Weak and Strong Patterns}

The sound patterns that we will use to illustrate the proposed approach can be placed on a continuum from weak to strong. Strong patterns are associated with phonological alternations involving fortition / strengthening processes, as well as non-alternating distribution patterns, and related diachronic changes that increase the range of phonemic contrasts in some context or within a language's overall inventory of sounds (see, e.g. Brandầ de Carvalho, Sheer \& Ségéral 2008). For the purposes of exposition we make the non-critical simplifying assumption that patterns that linguists refer to as fortition are generally associated with increased phonetic distinctiveness. For example, fortition, characterized by phonetic enhancement, occurs in Porteño Spanish where glides $/ \mathrm{j}, \mathrm{w} / \mathrm{strengthen}$ to homorganic obstruent fricatives in syllable-initial position (Lozano 1979). Conversely, weak patterns are associated with lenitions, deletions, or decreases in the range of phonemic contrasts as a result of sound merger in a given language (for reviews, see e.g., Gurevich 2011; Harris 2011; Kirchner 2001; Yu 2011). Likewise we assume that what linguists refer to as lenition is associated with reduced phonetic distinctiveness. An example of lenition can be found in the lenited pronunciation of $/ \mathrm{s} /$ as $[\mathrm{h}]$ in coda position in many varieties of Spanish (e.g., those spoken in the Americas; see Canfield 1981). ${ }^{2}$

\footnotetext{
${ }^{2}$ We note that this association between what linguists refer to as fortition/lenition may not always correlate with increases/decreases in phonetic distinctiveness in all languages in these ways.
} 
Cross-linguistically, strong and weak patterns typically align with certain contexts, as noted in Trubetzkoy's (1969[1939]: 240) seminal work on phonological relations. In his typological investigation into the nature of phonological neutralization, he discusses positions of maximal and minimal phoneme distinction; the former are contexts with a greater number of phonological contrasts, while the latter are contexts in which contrasts are neutralized. Similarly, Foley (1977:109) observes with regards to active phonological processes that "elements in strong positions undergo preferential strengthening, and elements in weak positions undergo preferential weakening." To foreshadow our arguments in the rest of the paper, we will show that language change processes tend to promote alignment of particular positions and sounds in ways that promote effective information transmission. Arguably as a result of such active processes over time, one of the hallmarks of typically strong contextual positions is that they tend to support a greater number of phonemic contrasts than do typically weak positions.

In the remainder of this section, we present the set of contexts associated with strong/weak patterns which, at least on the surface, is puzzlingly heterogeneous (e.g., Barnes 2006; Beckman 1998; Smith 2002, 2008; Zoll 2003). Some strong/weak patterns require reference to the domain of the lexical word for adequate description, while others are described with respect to the local segmental or prosodic level, such as patterns referring to syllable structure (e.g., Itô 1986). Still others seem to require reference to morpho-syntactic category, as in the common finding of preferential weakening of affix material in favor of root material (Casali 1997, 2011; Urbanczyk 2011).

Across the scientific enterprise, 'conspiracies', in which abstractly similar patterns appear in disparate contexts, often serve as hints of a more general causal unity (cf. Kisseberth 1970). Here, we will argue that these similar strong/weak patterns arise across disparate domain-types as a common phonological outcome of biases toward effective message transmission through the reduction of uncertainty. To begin, we review a sampling of strong and weak patterns that we find in these different types of domains. These patterns will form the empirical domain to which we return after introducing the proposed framework in more detail.

\subsection{Weak and strong patterns relative to the lexical domain}

It is well documented that many types of strong phonological patterns cluster toward the beginning of the word and conversely, weak patterns tend to be localized at the word end. In 
many languages (e.g. German, Turkish, Maltese), for example, voiced and voiceless obstruents contrast in non-word-final position but are neutralized word-finally where only the voiceless counterpart is attested (e.g. Trubetzkoy 1969 [1939]), e.g. German Hunde [hundə] / Hund [hunt] 'dogs, dog.' Word-final neutralization also affects length contrasts between geminate and singleton consonants: in Tiberian Hebrew; a long consonant occurs word-medially, while its short counterpart occurs word-finally, e.g. [11] [1], [qallos] 'light (fem)' / [qal] 'light (masc)' (Malone 1993). The asymmetry is also observed in Luganda where a root-initial geminate consonant is attested in word-initial position, while the corresponding singleton consonant occurs in non-word-initially, e.g. [ggi]/[ma-gi] ' egg' (class 5 sg./class 6 pl., Cole 1967). Similarly, in English syllable-initial voiceless stops are produced without aspiration in unstressed syllables, e.g. [ǽktəI] 'actor', except word-initially where aspiration is maintained, e.g. [thəméto] 'tomato'.

We likewise find that contrastive phonemic inventories are often larger word-initially and smaller toward the end of the word (reviewed in Beckman 1998; Kirchner 2001; Krämer 2007). In the native stratum of Turkish, for example, mid-round vowels are limited to the first syllable of words. In the Austronesian language Leti, geminate consonants and consonant clusters contrast in word-initial position, while word-finally, only single consonants or vowels are attested (Hume, Muller \& van Englenhoven 1997). Representative words are given in (1).

Leti

$\begin{array}{lllc}\text { ppikan } & \text { 'plate' } & \text { ptuna } & \text { 'star' } \\ \text { ttui } & \text { 'genre of literature' } & \mathrm{kd}^{\mathrm{y}} \mathrm{eli} & \text { 'ring' } \\ \text { kkusal } & \text { 'to be small' } & \text { vroan } & \text { 'axe' }\end{array}$

\subsection{Weak and strong patterns relating to morpho-syntactic category}

Weak and strong patterns can also be described by reference to the morpho-syntactic category in which they occur. Smith (2001), for example, refers to nouns as a strong lexical category: "they can either license more contrasts or resist more phonological processes than words of other categories can." More broadly, content morphemes cross-linguistically seem to behave as strong contexts relative to function morphemes (e.g., Trubetzkoy 1969 [1939], McCarthy \& Prince 1995, Urbanczyk 2011). As seen just above, for example, Luganda initial gemination only applies to root-consonants, not prefix consonants. Similarly, content morphemes, such as lexical 
roots, frequently trigger deletion in affixal material, but not vice versa. For example, Casali's $(1997,2011)$ cross-linguistic study of the patterning of sequences of two vowels $\left(\mathrm{V}_{1} \mathrm{~V}_{2}\right)$ shows that $\mathrm{V}_{1}$ deletion is typologically the common pattern, which he ascribes to the observation that VV sequences are typically generated through the concatenation of prefixes with stems; that is, it is the vowel of the prefix that preferentially deletes. Cases of $\mathrm{V}_{2}$ deletion are also observed though, as Casali points out, they generally occur when $V_{1}$ is part of a root and $V_{2}$ is part of a suffix or other function word. Finally, the set of function morphemes in a language often exhibits a restricted inventory of phonemic contrasts relative to the set that appear in content morphemes. Regular inflectional suffix consonants in English, for example, are overwhelmingly coronal, e.g.

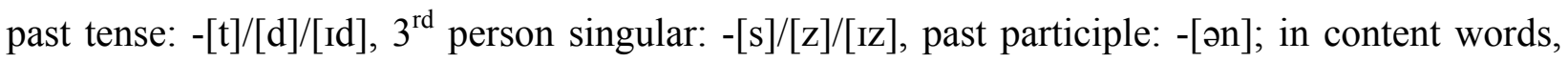
the full range of consonant place contrasts occur.

\subsection{Weak and strong patterns in the sub-lexical domain}

Contexts supporting weak and strong patterns are often defined sub-lexically with reference to prosodic or segmental structure. Prosodically, syllable onsets and stressed syllables tend to display strong patterns while syllable codas and unstressed syllables are commonly associated with weak patterns. The literature is replete with examples illustrating active phonological patterns that can be defined at the prosodic level (e.g. Selkirk 1982, Itô 1989). As noted above, in Porteño Spanish glides $/ \mathrm{j}, \mathrm{w} /$ are strengthened to homorganic obstruent fricatives in syllableinitial position (Lozano 1979). In English, voiceless stops are aspirated when onset-initial in stressed syllables. Similarly, a greater number of phonemic contrasts tends to be associated with prosodically strong contexts, while fewer contrasts are observed in weak contexts. In most varieties of English, many vowel contrasts are only found in stressed syllables regardless of position within the word, while in unstressed syllables, the vowel inventory is limited. Likewise, in Copala Trique (Hollenbach 1984), the final syllable of a word is predictably stressed, supporting eight phonemically contrastive tone patterns, while only two to three tone patterns occur on non-final syllables.

Segmentally-defined weak and strong contexts display similar asymmetries. In intervocalic consonant clusters $\left(\mathrm{VC}_{1} \mathrm{C}_{2} \mathrm{~V}\right.$, e.g. abdo $), \mathrm{C}_{1}$ is typically considered to be in a weak position since it often undergoes processes that neutralize phonemic distinctions. A common case involves nasal consonants $(/ \mathrm{m}, \mathrm{n}, \mathrm{y} /)$ that assimilate to the place of articulation of a following consonant, 
thereby losing their own distinctive place of articulation. In English this can be observed as a variable process across morpheme and word boundaries, e.g. brow $[n]$ bag $\sim$ brow $[\mathrm{m}]$ bag (e.g. Dilley \& Pitt 2007), or as a static distributional pattern within words, e.g. u[mb]rella, u[nd]er, si[ng]le. Conversely, consonants in $\mathrm{C}_{2}$ position typically preserve or enhance their distinctions from other consonants in this position (Jun 2011). The behavior of $\mathrm{C}_{2}$ in nasal place assimilation is consistent with this pattern given that such consonants preserve their own place of articulation, while at the same time triggering assimilation. Such patterns generally lead to the inventory of consonants in $\mathrm{C}_{1}$ position being smaller than that in $\mathrm{C}_{2}$ position. This can be seen in Japanese, for instance, where consonants in $\mathrm{C}_{1}$ position lack a distinctive place of articulation; rather, nasal consonants are homorganic with a following consonant and obstruents occur only as geminates. No such restrictions hold for $\mathrm{C}_{2}$ consonants (Itô 1989).

\subsection{Summary}

Similar weak and strong patterns are defined over disparate domain-types, including word, morpho-syntactic category and sub-lexical domains. Looking forward, we will propose that this 'conspiracy' is grounded in the fact that these strong/weak patterns reflect contributions to message transmission accuracy, and that contexts defined over each of these domains can be predictably associated with a greater or lesser contribution of a PUC to message transmission accuracy (though, like any bias operating over a complex system in which multiple biases jointly affect the outcome, this association is gradient). If weak and strong patterns reflect a trade-off between accurate information transmission and lower resource cost, strong patterns should occur in contexts in which the communicative advantage of increased phonetic cue distinctiveness typically outweighs its disadvantage in cost (see $\$ 3.6$ regarding cost). Conversely, weak patterns should arise in contexts in which investment in phonetic cue distinctiveness contributes relatively little to message transmission accuracy. Before elaborating the approach further, we review basic concepts relating to message transmission systems and how these concepts allow us to make predictions about phonological patterns and change in linguistic sound systems. 


\section{A Model of Message Transmission}

The whole problem of efficient and error-free communication turns out to be that of removing from messages the somewhat inefficient redundancy which they have and then adding redundancy of the right sort in order to allow correction of errors made in transmission.

John Pierce 1960: 164

A core motivation of the present approach arises from the fact that human language, in its role as a message-transmission system, is subject to the same theoretical constraints that govern message-transmission systems in general (Shannon 1948; also Nowak \& Krakauer 1999; Plotkin \& Nowak 2000). That language is such a system is not controversial; rather, a central question has been whether factors in message transmission play a role in actively shaping linguistic representations, including phonological systems (see, e.g. Hockett 1955 in support of such an approach, Chomsky 1957 against such an approach, and Pierce 1980 for a rebuttal of Chomsky's criticisms of information-theoretic approaches to linguistics). While, as noted in Section 1, many previous researchers have identified apparent links between phonological patterns and their role in communication, new advances in theory, experimental technologies, and data analysis are allowing researchers to examine these links in increasingly sophisticated ways. In particular, the availability of larger datasets allows linguists to draw conclusions from statistically significant patterns rather than single examples. The aggregate evidence from these investigations indicates that variation in spoken language is biased toward lower resource cost as well as higher message transmission accuracy (for a recent review, see Jaeger \& Buz, in press) ${ }^{3}$ and that the tension between these biases influences the trajectory of sound change over time in a speech community. Our goal is to illustrate how mathematical frameworks for communication allow us to integrate these biases into a theory of phonology. In this section, we provide an informal introduction to the relevant concepts in formal theories of communication.

\footnotetext{
${ }^{3}$ It is a reasonable assumption that other modes of language are also subject to the same biases. Since our focus is on sound patterns, we limit our discussion to spoken language (for related evidence that speakers seem to take advantage of the visual modality to achieve this trade-off, see, e.g., Cook et al., 2009; Pate \& Goldwater, 2015).
} 


\subsection{Language as a system of message transmission}

Human languages are message transmission systems: an intended message is encoded into a signal which is transmitted from the source to a recipient, who then decodes it back into a message. Information theory (Shannon 1948) provides us with mathematical tools to understand such systems. When the decoded message at the receiver is the same as the message encoded by the sender, we refer to accurate transmission. At the system level, we can ask how much a language on average-i.e., across all its users and all different situations-supports accurate message transfer. We refer to this property of a language as (communicative) robustness (for a recent review of this notion and its relevance for sound systems, see Winter 2014). Message transmission systems are more robust to the extent that they support more reliable message transmission over the diverse situational and environmental contexts in which they are used. ${ }^{4}$

\section{(2) Robustness}

A robust communication system is one that is able to support reliable, i.e. low error, message transmission under typical conditions.

Why be robust? In part because transmission error itself incurs cost: when a linguistic communication error is detected by interlocutors, it requires additional expenditure of effort for recovery, or it can lead to more lasting misalignment between interlocutors. However, robustness does not come for free either. As we discuss in Section 3.6 greater robustness typically implies greater resource cost, so that robustness is not the only factor under consideration. That is, robustness trades off with other system properties. The current proposal is guided by the growing body of evidence indicating that the trade-off between message transmission accuracy and such costs influences the structure of phonological systems. In the rest of this section we provide an intuitive introduction to the relevant formal concepts from information theory (Shannon 1948) and probabilistic inference (Bayes 1763; Laplace 1886), as well as a review of the empirical grounding of these concepts for human language use. These tools provide a way of understanding how robustness can be balanced relative to resource cost to create an effective system of

\footnotetext{
${ }^{4}$ Robustness is also sometimes used to refer to graceful decrease in performance under adverse conditions (e.g., in robotics). Human language use in communication arguably has this property, too, although our focus here lies elsewhere.
} 
information transmission, as stated in (3), and by extension, provide a basis for generating tests of the general approach.

\section{(3) Effective Message Transmission}

A message transmission system is effective when system biases toward robustness trade off with resource cost.

\subsection{What is the signal? And what is the message?}

Spoken language has hierarchical structure, carrying information of nested, abstract categories such as word, phoneme, and feature. We can think of the acoustic signal as a 'mid-point' in the many stages of encoding and decoding between the speaker and hearer. Beginning with interaction with physical structures of the ear, the acoustic signal is progressively transformed through interaction with the processing apparatus, where each of these transformations can itself be considered a form of message transmission (cf. Jaeger 2010, pp. 50-51). As a consequence, for the purposes of discussion we need to specify what part of this transmission process we will be focusing on as 'the signal', and what as 'the message'.

Here, we will use the term 'signal' to refer to the stage in linguistic signal processing in which a decomposition into phonetically relevant properties has already been imposed. At this level, the processing system has extracted structured yet gradient properties of the signal (e.g., Mesgarani et al. 2014) such as VOT, formant value, or fricative center of gravity. We focus on this particular portion of the long relay from acoustic signal to linguistic message because it provides the greatest contact with existing phonological research.

In theory, these phonetic cues could contribute to transmission of a message defined at any categorical level, such as a feature, a phoneme or a word. For example, perceiving a long VOT might help a listener decide that an initial stop is a /p/ rather than a /b/, while at the same time it might help the listener decide that a word is pat rather than bat. For sound-centric approaches to phonology - those that account for (morpho-)phonological patterns without taking into account the transmission of meaning-bearing units - the recognition of abstract phonological categories from gradient properties of the acoustic signal is the primary or even only stage in processing that is relevant for phonological explanation. In such views, for example, a word-final consonant may reduce because it has weak cues to its identification. In contrast, a central argument of the 
approach advanced here (MOP) will be that phonological patterns cannot be fully understood without recognizing that biases toward effective transmission of meaningful messages (including semantic, pragmatic, and social meaning) strongly affect the shape of these sound patterns. From this perspective, the probability of reduction of a word-final consonant is related to the probability that the message (e.g. word), rather than only the consonant, will be correctly recognized. For the purpose of our argument, we can think of two inference processes that are critical to phonology. The first is the one that sound-centric approaches focus on: given the properties of the acoustic/auditory signal, how likely is a listener to accurately perceive a phonological unit in context or PUC, e.g. a pre-vocalic consonant or a word-initial onset? We focus on a second inference process in this paper because we think that its consequences for phonology have been underestimated. This concerns the mapping of PUCs to meaning-bearing units (i.e., messages):

(4) Given a PUC that can be accurately perceived, how much does it contribute to accurate message transmission?

The nature of phonological variability, sound change, and the shape of phonological systems cannot, we propose, be understood without also taking into account a PUC's contribution to information transfer. Therefore, in the remainder of this section we focus on meaning-bearing units as the messages and their role in effective communication.

\subsection{Uncertainty about the intended message}

In the mapping from signal to message, there is always some degree of uncertainty associated with the intended message. In this section we introduce two general sources of uncertainty, noise and inherent ambiguity, which encompass a variety of distinct phenomena that contribute to accurate message transmission. The joint effect of noise and ambiguity on uncertainty is illustrated in Figure 1. With this as a basis we turn to Shannon's (1948) solution to the problem of resolving uncertainty. This, we suggest, provides key insights into understanding the shape of phonological patterns. 


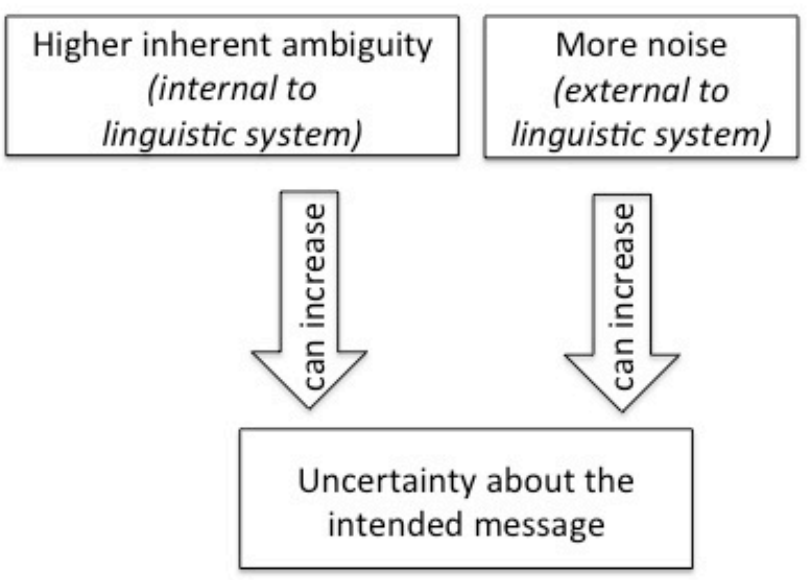

Figure 1: General sources of uncertainty about an intended message

Inherent ambiguity refers to the degree to which multiple messages can compete for identification with the same signal (e.g. Jurafsky \& Martin 2000). This competition is a function of overlap in the distribution of signals that are associated with different messages, and is jointly specified by the lexicon and the phonological grammar. For example, the expected signal for the intended message CAT will bear some degree of perceptual similarity to expected signals for lexical neighbors, such as CAP, where the degree of ambiguity is influenced by the details of the given signal as decoded by the receiver (e.g., for a review of lexical neighborhood effects in processing, see Chen \& Mirman 2012; Luce \& Pisoni 1998; Magnuson et al. 2007). Ambiguity is gradient, and in principle any given signal has some non-zero probability of being mapped to any possible message. ${ }^{5}$

Noise here refers to any unpredictable variation in the production, transmission, perception or processing of the signal. In this sense, noise is external to the linguistic system and, for the purpose of linguistic theory building, not a property that phonologization can directly affect. Noise is introduced by diverse factors ranging from intrinsic variation in neural firing rates (Shadlen \& Newsome 1994; Tolhurst et al. 1983), to environmental noise such as static in a

\footnotetext{
${ }^{5}$ Note that it does not follow that ambiguity in the mapping of signal units to message is always a bad property of a communicative system. First, it is not always detrimental because parts of the signal that are ambiguous out of context can be disambiguated by context. Second, it can also be beneficial because ambiguity means that parts of the signal that have particularly desirable properties for other reasons (e.g., particularly easy-to-plan and articulate phonemes or words) can be reused more frequently, thereby lowering the cost to a language. Taken together, this means that ambiguity can increase the communicative utility of the language (see Piantadosi et al., 2012).
} 
phone line. When unpredictable variation is greater, inaccurate message transmission is more likely. While there are many different sources of noise, each of them differing in important aspects, ${ }^{6}$ they all introduce unpredictable variation into the signal and thus uncertainty about the intended message. This consequence is shared with ambiguity, which-for ontologically different reasons - also introduces uncertainty about the intended message. A critical consequence of this for our purposes here is that there is always some uncertainty about the intended message.

Shannon (1948: 22) showed that uncertainty in the signal-message mapping can be reduced by manipulating redundancy in the signal. In information theory, a redundant signal is one which contains detail beyond that which is necessary for accurate message transmission under ideal (noise-free) conditions (Pierce 1980).

(5) Redundancy (based on Pierce 1980: 292)

A redundant signal contains detail beyond that which is necessary for accurate message transmission under ideal conditions.

In general, greater redundancy in cues to the identification of a message increases the probability that the receiver will reconstruct an intended value. Two sources of redundancy are relevant for our discussion (see also e.g., Aylett \& Turk 2004, 2006). First, message predictability refers to detail contained in the context-for example, in the form of additional lexical or functional material - that influences the likelihood that the signal maps onto the intended message. The second, signal specificity, refers to detail in the signal that makes the signal more likely to have been generated for the intended message rather than for some other message. ${ }^{7,8}$ Note that in this

\footnotetext{
${ }^{6}$ For example, different types of noise have different structures-i.e., they differ in what aspects of the signal they transform and in what way (e.g., via adding, subtracting, or warping of the signal). They also differ with regard to their systematicity (e.g., a high frequency sine tone vs. multi-talker background noise). Both of these differences can affect what specifically constitutes an effective solution to the presence of such noise. For now, we abstract away from this detail, as our primary focus here is on establishing the foundations of the proposed framework.

${ }^{7}$ Our term message predictability is related to "language redundancy" in Aylett and Turk (2004). The use of message predictability highlights our hypothesis that the language redundancy that is relevant to phonology is ultimately meaning-based, rather than being based on linguistic forms (though form-based estimates are often used in investigating this hypothesis, as they are easy to obtain). Signal specificity is related to "signal redundancy" in Aylett and Turk (2004). Here we prefer the term "specificity". Whereas "signal redundancy" suggests a single dimension of redundancy, the term specificity highlights that
} 
usage anything that increases a signal's specificity is understood to be an increase in the signal's redundancy. Signal redundancy can be increased either by the addition of new phonetic cues to message identity, or the enhancement of an existing cue.

Consistent changes in signal specificity can generate patterns that are eligible to be phonologized over time. Changes in message predictability, on the other hand, are changes to the system of messages themselves, e.g., changes in the syntagmatic or paradigmatic contexts in which signals occur. The combined effect of message predictability and signal specificity on uncertainty is illustrated in Figure 2 and stated in (6).

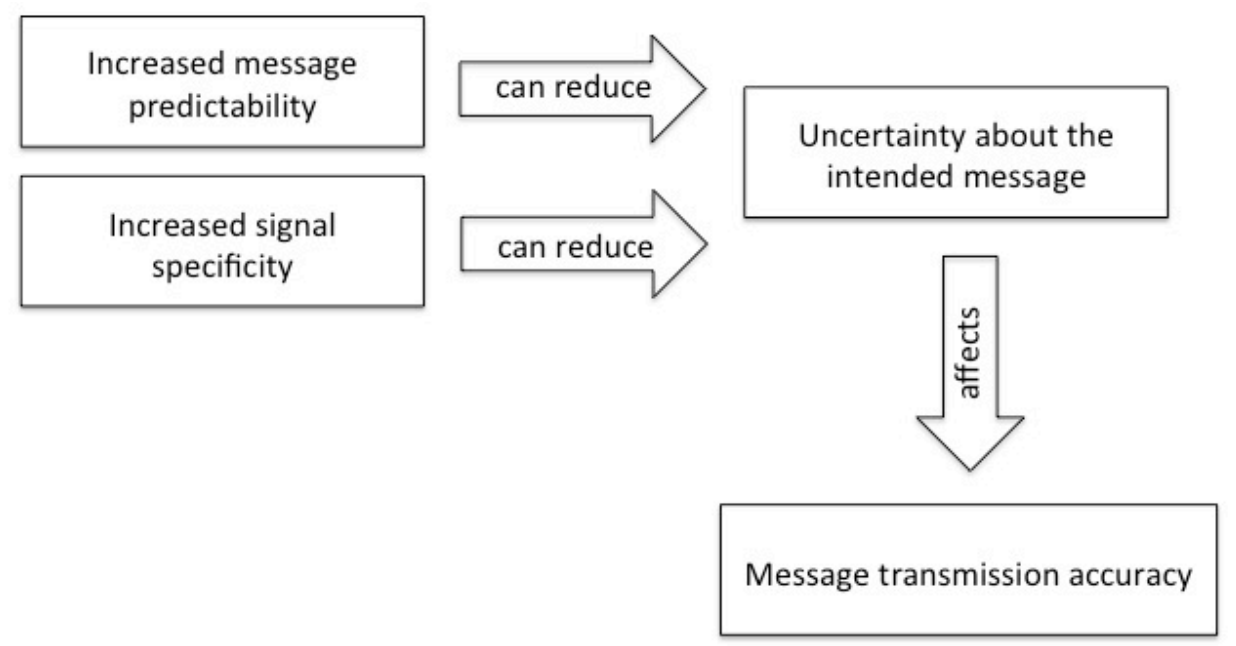

Figure 2: Combined effect of message predictability and signal specificity on message uncertainty and message transmission accuracy

Increasing redundancy can increase the range of conditions over which a message can successfully be recovered, reducing uncertainty and hence contributing to greater system robustness. This role for redundancy is familiar to phonologists: lexical and phonological categories are signaled by multiple phonetic cues (e.g., Repp 1982; Lisker 1986; Whalen et al. 1993; see also Beddor 2009; Toscano \& McMurray 2010), and listeners are able to integrate cues to accurately reconstruct an intended category even when some cues are degraded, missing, or

phonetic signals fall into a multi-dimensional perceptual space, and that it is the (average) distance of signals in this space that should affect the accuracy of message transmission accuracy.

8 'Inherent ambiguity' as described above can thus be thought of as the inherent 'signal specificity' of a message. To avoid confusion, we use the term 'signal specificity' only to reference token-level properties of signals. We use the term 'inherent ambiguity' to refer to the expected overlap between the distribution of the message's signals with the distributions of other message' signals. 
displaced (e.g., Beddor 2009; Ganong 1980; Repp 1982). Just as there is always some uncertainty in the mapping from a signal to an intended message, there is always some redundancy that can be introduced into the system to reduce that uncertainty further (Shannon 1948). However, as we discuss in Section 3.6 below, increases in redundancy may come at the price of increased resource cost, such that effective message-transmission systems target redundancy where it reduces uncertainty most cost effectively.

\section{(6) Uncertainty, message predictability and signal specificity}

Message predictability and signal specificity jointly influence accurate message transmission: the greater the predictability of a message and the more specific the signal is to the intended message, the less uncertainty there will be about the outcome being the intended message, all else being equal.

In the next section, we examine the problem of resolving uncertainty in the mapping between a signal and an intended message in the context of Bayes' Theorem, showing that by taking into account message predictability and signal specificity we are able to generate predictions about how language users manipulate redundancy in response to uncertainty.

\subsection{Uncertainty and Bayesian inference}

Uncertainty makes language comprehension, including speech perception and the recognition of phonological sequences or words, a problem of inference under uncertainty. As we will see, acknowledging this leads the way to a mathematical framework to reason about how the message shapes phonology through the relationship between redundancy and accurate message transmission. Inference under uncertainty is a computational problem, and in this section, we discuss how Bayesian inference allows us to specify ideal solutions to this problem. These ideal solutions, also known as "ideal observers", ${ }^{9}$ have played and continue to play an influential role in the cognitive sciences (for a recent review, see Clark 2013). This includes research on speech

\footnotetext{
${ }^{9}$ Ideal observers are not to be confused with the ideal speaker/hearer (Chomsky 1965), which has been influential in linguistic argumentation. Whereas the "ideal speaker/hearer" is more accurately described as idealized speaker/hearer, ideal observers are optimal mathematical solutions to perceptual inference problems (sometimes ideal observer models make idealized assumptions, such as the absence of memory constraints; still, for something to be an ideal observer in the sense intended here, it needs to define an optimal solution to a specified problem under whatever assumptions are made).
} 
perception (for an introduction, see Weatherholtz et al., submitted) and higher-level language processing, to which we return below (for introduction and review, see Kuperberg \& Jaeger 2016).

High message predictability limits the potential contribution of the signal to reducing uncertainty in message transmission. For example, in the limit in which there is only one possible message that could be conveyed in the current context (i.e., there is extremely high message predictability), the signal itself could be entirely non-specific, and the message could still be transmitted without error. Similarly, we can conceptually hold message predictability constant and think about how signal specificity can influence the probability of accurate message transmission. To provide an intuitive example, consider the /u/-containing English words boot and prove. There are many English words of the form /b_t/, such as boat, bat, but ..., but only one word of the form $/ \mathrm{pr}_{-} \mathrm{v} /$. As a consequence, if a hearer accurately hears cues to the local phonological context /pr_v/, noise that interferes with phonetic cues to the identity of the vowel will have relatively less effect because the cues for /pr_v/ within the signal leave little residual uncertainty. Conversely, even if a hearer accurately perceives cues to the local phonological context $/ b_{-} t /$, noise that interferes with phonetic cues to the identity of the vowel interferes relatively more with recognition of the word, because there are many other lexical competitors with similar signals. All else being equal, increasing signal specificity by adding or enhancing existing cues to the vowel PUC (i.e., increasing its redundancy) reduces uncertainty about the intended message boot more than prove.

Now that we have established the basic intuition about how message predictability and signal specificity jointly affect successful recognition, we turn to Bayes Theorem, which provides a formalization of these intuitions in terms of probability theory. Clark (2013), for example, in his "Bayesian Brain Hypothesis" reviews evidence that "the nervous system is fundamentally adapted to deal with uncertainty, noise, and ambiguity, and that it requires some (perhaps several) concrete means of internally representing uncertainty," which he argues is best modeled in terms of Bayesian prediction of upcoming events (2013: 188). Indeed, Bayesian inference has been applied successfully to a variety of problems in speech production (e.g., phonetic imitation, Nielsen \& Wilson 2008; hyper-articulation, Buz \& Jaeger, in prep; Kirov \& Wilson 2013) and perception (e.g., the perception of similarity, Feldman et al. 2009; phonetic categorization, Clayards et al. 2008; auditory-visual cue integration, Bejjanki et al. 2011; 
compensation for co-articulation, Sonderegger \& Yu 2010; phonetic adaptation, Chodroff, Golden, \& Wilson, in prep; Kleinschmidt \& Jaeger 2015; spoken word recognition, Norris \& McQueen, 2008). Bayes' theorem is useful to us because it allows us to understand uncertainty as a specific function of the product of message predictability and signal specificity, as shown in Equation (E1) (see e.g., Flemming 2010; Jaeger 2010b; Wedel et al. 2013b). Note that message predictability and signal specificity are both normalized by the denominator in E1.

In Equation (E1), $M$ is any message to be transmitted. $S$ is the signal that is used for that message during a particular transmission event, and ctxt is the context in which the transmission takes place. The right-hand side of the equation, $p(M \mid S, c t x t)$, is the probability of accurate message transmission, that is, the probability that this particular message will be correctly identified by the listener, given the use of a particular signal in a particular context. In Bayesian terminology, this is considered the posterior probability of the message. It is this posterior distribution that an ideal observer draws on when making inferences about what message was produced.

(E1) Probability of accurate communication as a function of message predictability and signal specificity:

\begin{tabular}{|c|}
\hline Message \\
Predictability: \\
Probability that this \\
message, as opposed \\
to any other \\
message, is sent, \\
given the context. \\
\hline
\end{tabular}

Signal Specificity:
Probability that
this particular
signal is used for
this message, as
opposed to any
other message,
given the context.

\begin{tabular}{|c|}
\hline Probability of \\
Accurate Message \\
Transmission: \\
Probability that the \\
message is accurately \\
received, given the \\
signal and context. \\
\hline
\end{tabular}

$$
\frac{p(M \mid c t x t) * p(S \mid M, c t x t)}{\sum p\left(M_{i} \mid c t x t\right) * p\left(S \mid M_{i}, c t x t\right)}=p(M \mid S, c t x t)
$$

Bayes theorem also tells us that the posterior distribution is determined by two other distributions, (i) the prior probability of the different messages that are possible in the current context, $p(M \mid c t x t)$, and (ii) the likelihood of observing a given signal given the intended message and the current context $p(S \mid M, c t x t)$. These two distributions relate to the two intuitive concepts we introduced at the beginning of this section, as shown in Equation (E1). First, the 
predictability of a message in a context corresponds to its prior in that context. Second, the specificity of a signal is captured as the likelihood term, $p(S \mid M$, ctxt $)$, i.e., the probability that the particular signal, $S$, will be used if the message is $M$ given the particular context. In order for the posterior probability distribution to be well-formed, both the prior and the likelihood term need to be normalized. This is achieved by the denominator, which sums the probabilities for the signal $S$ over all possible messages and their respective prior probabilities, giving the overall probability of the signal $S$ for some message given the context.

The normalization through the denominator provides for the intuitive result that adding redundancy to increase signal specificity can reduce uncertainty and thus increase message transmission accuracy - even if the resulting variant is not in fact the most often-encountered signal for that message. To illustrate this, consider the utterance, I really like my cat. In rapid, casual speech, American English speakers often use highly reduced stop allophones utterancefinally, so a signal for cat in this context often will provide only weak acoustic cues to the identity of the final stop (see Section 3.6 for why redundancy would be reduced in order to reduce resource cost). This reduced signal for the message cat may be relatively frequent, but its signal specificity is low; a similar acoustic signal is, for example, also plausible for an intended message cap. In comparison, a hyperarticulated variant such as $\left[\mathrm{k}^{\mathrm{h}} \mathfrak{x} \mathrm{t}^{\mathrm{h}}\right]$ may be less often encountered for the message cat (thus making $\mathrm{p}(S \mid M$, ctxt) relatively low), yet this clearer signal is nonetheless more likely to be accurately identified with the message cat, especially in contexts in which the probability of cat is lower. The source of this dissociation of accuracy and probability of signal occurrence lies in the denominator, which accounts for the chance that the signal could be associated with any other message in this context. The reduced signal with weaker cues to the final stop is relatively consistent with both the messages cap and cat, and so is specific for neither. In contrast, the probability of hearing the hyperarticulated signal $\left[\mathrm{k}^{\mathrm{h}} æ \mathrm{t}^{\mathrm{h}}\right]$ if the message were not in fact cat is low, so despite being a relatively infrequent signal for the message cat, it is more specific.

The relationship between message predictability and signal specificity provides predictions relevant to phonology about the situations in which increasing signal redundancy can facilitate accurate message transmission, and conversely, when signal redundancy can be reduced. The phonological grammar of a language describes a probability distribution over signals for possible lexical representations. Stated in terms of traditional phonology, given some underlying 
lexical representation the grammar predicts a phonologically expected form mapping to the expected values of the phonetic cues making up the signal, given the phonology. All else being equal, productions of a word should tend toward the phonologically expected. If that form would have particularly low specificity given its predictability in some context, the MOP framework predicts speakers will be biased toward producing a more redundant variant than expected, in order to support accurate message transmission, $\mathrm{p}(M \mid S, c t x t)$. Conversely, a relatively reduced form is predicted if the phonologically expected form would correspond to an unnecessarily high-specificity signal given the context.

As we will review next, phonetic and psycholinguistic evidence suggests that speakers are in fact biased toward pronunciations that increase signal specificity when it is more likely to support message transmission accuracy. Where this bias toward greater signal specificity is consistent across similar lexical items, MOP predicts that this may be phonologized into a regular pattern across the language. In Section 4 we present evidence that the range of phonologized patterns within and across languages is consistent with predicted interactions of message predictability and signal specificity.

\subsection{Modulating redundancy in response to uncertainty}

Like all real-world communication systems, language is characterized by uncertainty that arises through signal ambiguity, and through noise that degrades signal distinctions. Moreover, like communication systems more generally, redundancy plays a role in adapting to varying levels of uncertainty. Degree of redundancy is not a static property of a language system; rather, a growing body of work suggests that redundancy is modulated in speech in a way that supports message transmission accuracy in context (see e.g., Aylett \& Turk 2004, 2006; Frank \& Jaeger 2008; Kurumada \& Jaeger 2015; Pate \& Goldwater 2015; van Son \& Pols 2003; for recent discussions, see Ernestus 2014; Jaeger \& Buz in press). ${ }^{10}$ As hypothesized in Lindblom's (1990) seminal hyper- and hypo-articulation model, both laboratory and natural speech-based evidence shows that phonetic redundancy is modulated depending on the predictability of a

\footnotetext{
${ }^{10}$ While these correlations are well documented, it is an on-going debate as to whether the signal is strategically manipulated on the part of the speaker with the goal of achieving better communication (e.g., Jaeger 2013; Buz et al., 2016; Lindblom 1990; Schertz 2013; Stent et al. 2008) and/or to some other mechanism, such as filtering in comprehension (e.g., Blevins \& Wedel 2009; Guy 1996; Ohala 1988; Pierrehumbert 2001, 2002; Wedel 2006, 2012) or effects of lexical planning on articulation (e.g., Arnold et al. 2012; Baese-Berk \& Goldrick 2009; Bell et al. 2009). We return to this issue in Section 5.2.
} 
word in its local context: phonetic substance is added with lower word (or syllable) predictability and decreased with higher predictability (e.g., Aylett \& Turk 2004, 2006; Bell et al. 2003, 2009; Cole et al. 2010; Jurafsky et al. 2001; Kuperman \& Bresnan 2012; Pluymaekers et al. 2005a; Raymond et al. 2006; Rose, Hay \& Hume 2016; Tily et al. 2009; Tily \& Kuperman 2012; Torreira \& Ernestus 2009; Seyfarth 2014; van Son \& Pols 2003; van Son \& van Santen 2005; for review, see Jaeger \& Buz, in press). ${ }^{11}$ More predictable words are also comprehended more quickly (in speech: e.g., Grosjean 1980; Tyler \& Wessels, 1983; in reading: e.g., Frank \& Bod 2011; MacDonald \& Shilcock 2003; Smith \& Levy 2013) and recognized more accurately in noise (e.g., Obleser et al. 2007). In particular, even phonetically-reduced forms are easily recognized if embedded in supportive contexts (Ernestus 2014; Ernestus, Baayen, \& Schreuder 2002; see also Mitterer \& McQueen 2009; Sumner, Kurumada, Gafter, \& Casillas 2013). Further, there is a growing body of evidence suggesting that uncertainty introduced through competition from messages with perceptually similar signals influences production (e.g, Munson 2007; Gahl \& Strand 2016; Buz \& Jaeger 2016; Wedel, Nelson \& Sharp in prep) ${ }^{12}$ as well as comprehension (e.g., Luce \& Pisoni 1998; Magnuson et al. 2007; Chen \& Mirman 2012, 2014), though these effects remain less well-understood than the effects of predictability. Recent evidence from laboratory studies suggests that a phonetic cue to lexical identity tends to be hyperarticulated when that cue contributes strongly to disambiguating the word from a minimal pair neighbor that is contextually available (Baese-Berk \& Goldrick 2009; Buz, Tanenhaus, \& Jaeger 2016, in prep; Peramunage et al. 2011; Kirov \& Wilson 2012; Schertz 2013; Seyfarth, Buz, \& Jaeger 2016).

For example, Seyfarth, Buz, \& Jaeger (2016) asked speakers to produce a word-final /s z/ contrast in the presence of a contextually-salient minimal pair competitor, e.g., dose $\sim$ doze. Participants produced one of three words displayed on a computer screen for a confederate who

\footnotetext{
${ }^{11}$ A related line of research has found that more predictable syntactic structure seems to be correlated with reduced prosodic phrase boundaries, as reflected in less phrase-final lengthening (e.g., Gahl \& Garnsey, 2004; Gahl et al., 2006; Kurumada, 2011; Turk 2010).

${ }^{12}$ Here we focus on research in production that has investigated the effects of neighborhood structure on the phonetic realization of words (see also Munson \& Solomon 2004; Scarborough 2012, 2013; Wright 2004, which however all are targeted at another question and confound neighborhood structure and frequency effects). A related line of research has investigated the time course of lexical production (for review, see Sadat et al. 2014) and the likelihood and types of speech errors as a function of neighborhood structure (e.g., Dell, Schwartz, Martin, Saffran, \& Gagnon 1997; Goldrick et al 2010; Stemberger 2004; for reviews, see Sadat et al. 2014).
} 
had the task of choosing the same word on a screen. Half of the presentations included the minimal pair competitor as one of the other words on the screen, and half did not. Two different phonetic cues to the word-final $/ \mathrm{s} \sim \mathrm{z} /$ contrast were found to be significantly hyperarticulated in the presence of a competitor: vowel length (shorter for $/ \mathrm{s} /$, longer for $/ \mathrm{z} /$ ), and the perseveration of voicing into $/ \mathrm{z} /$. This supports the idea that speakers are able to manipulate phonetic cues to increase perceptual distance to specific competitors (e.g., Wedel et al. in prep; see Buz \& Jaeger, in prep, for evidence that this and similar forms of hyperarticulation indeed facilitate more accurate comprehension).

Similar but less pronounced hyperarticulation of phonetic cues that distinguish words from minimal pair neighbors has also been observed in laboratory studies even when the minimal pair competitor is not present in the context (Baese-Berk \& Goldrick 2009; but see Goldrick et al 2013, Fricke et al 2016; for discussion, see Buz \& Jaeger 2016; Fox et al. 2015). Similarly, evidence from conversational speech shows that phonetic cues which distinguish a word from a lexical minimal pair are hyperarticulated in a way that increases perceptual distance from that competitor, even when the competitor itself does not appear in the discourse (Nelson \& Wedel submitted, Wedel et al in prep.). In these studies, the VOT of voiced word initial stops was found to be significantly shorter for words with a voicing minimal pair (e.g., bat $\sim$ pat) than for words without a voicing minimal pair (e.g., badge *padge), as shown in Figure 3. Conversely, the VOT for voiceless word initial stops was significantly longer for words with a voicing minimal pair (e.g. tie $\sim$ die) than for words without a voicing minimal pair (e.g., test $\sim * d e s t)$. The observation of greater VOT for voiceless stops, versus shorter VOT for voiced stops suggests that VOT is modulated in a way that creates greater signal specificity relative to a lexical alternative. Similarly, in the same dataset vowels were found to be shifted in formant space specifically away from nearby competitor vowels if they defined a lexical alternative, (e.g. ship sheep). 

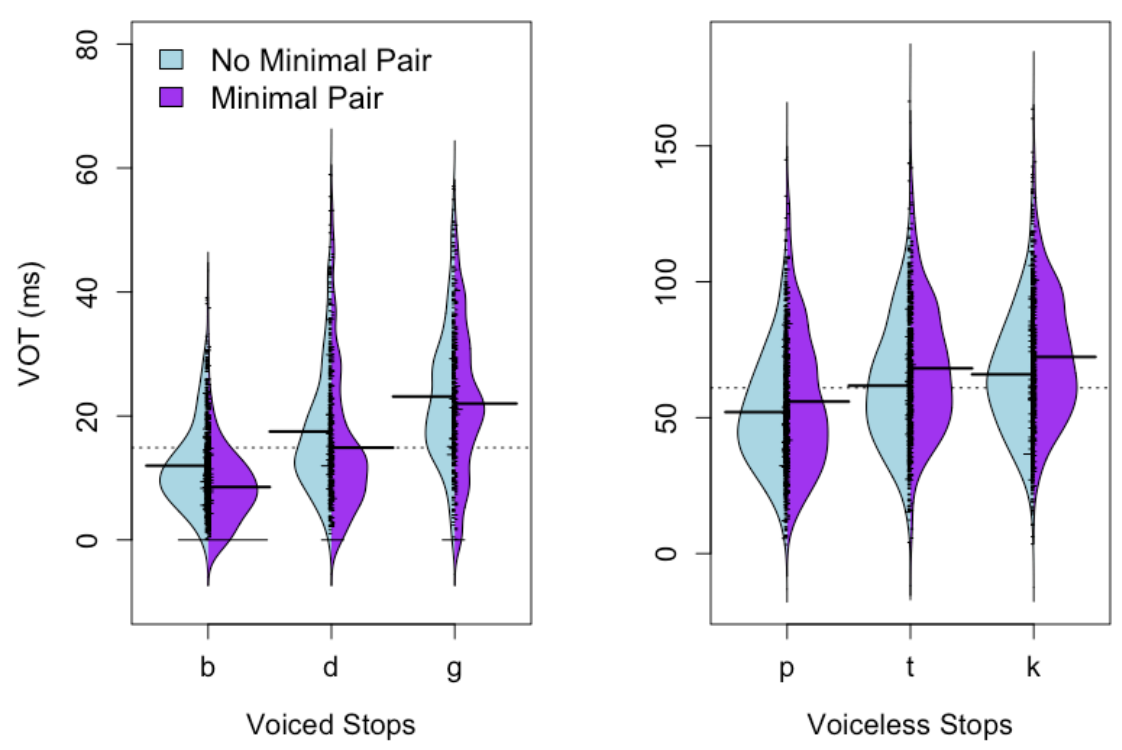

Figure 3. Plots of VOT by minimal pair competitor existence for voiced and voiceless stops in the Buckeye Corpus of American English (Pitt et al. 2007). Black horizontal lines within the plots show the mean VOT values for each condition. The horizontal dotted lines in each plot indicate the group means for all three places of articulation. Reprinted with permission from Wedel et al. (in prep).

Finally, there is also evidence that more generally, sounds that cue lexical contrasts are hyperarticulated relative to those that cue more allophonic / predictable distinctions (e.g., Gick et al. 2006, Cristia \& Seidl 2013, Hall et al. 2014) - for example, Hall et al. (2014) show that there is greater tongue movement in tense vowels in English when they are in a context where they are in lexical contrast with lax vowels than when they are in an environment where their contrast with lax vowels is neutralized. In Section 4.3 below, we review evidence that such word-level hyperarticulation affects the development of phonological patterns.

\subsection{Balancing robustness and resource cost}

The results reviewed above are consistent with the hypothesis that the speech signal is modulated in response to the expected uncertainty about the intended message. However, if adding redundancy can always increase the probability of the message being conveyed accurately, one might ask why we don't simply add unlimited redundancy to maximize the probability of accurate message transmission in any context. As noted in $\S 3.1$, the answer lies in 
the observation that increasing redundancy conflicts with other inter-related properties beneficial to systems of information transfer, such as fast message transmission (speed) and metabolically cheap message transmission (effort), which includes efficient use of limited cognitive resources. Similar to the limits imposed by an internet provider on up- and download rates, we can think of any communication system in terms of the number of messages that are conveyed in a certain amount of time (i.e., the rate of information transmission, e.g, Levy \& Jaeger 2007; Pellegrino et al. 2011) or for a certain amount of effort (e.g., Lindblom 1990; Zipf 1949). There are thus typically increased costs to a more robust communicative system (see also Zipf 1932; Ferrer i Cancho \& Díaz-Guilera 2007; Jaeger 2006, 2013; Piantadosi et al., 2012; Pate \& Goldwater, 2015). As an example of the relationship of cost and redundancy, increasing the redundancy of a phonetic signal to make it more specific to the intended message may involve adding new phonetic cues or enhancing those already present (or reducing the variance around an articulatory or acoustic target, cf. Buz \& Jaeger, in prep), while increasing contextual predictability of the message can involve providing more discourse cues in the form of additional lexical or functional material. In these examples, increasing redundancy involves increased resource cost on the part of the speaker, which decreases overall system effectiveness.

We are not the first to propose that 'resource cost' is an important factor in understanding the phonetic grounding of phonology (e.g., Cohen Priva 2012; Flemming 1997; Gafos 2002; Hayes 1999; Kirchner 2001; Lindblom 1990; for a recent discussion of the potential pitfalls of 'cost', see Pouplier 2012). The notion of 'resource cost' covers a broad and heterogeneous set of potential influences on speech at multiple levels of analysis. For example, some proposals focus on metabolic costs (e.g., the metabolic costs of motor movements during articulation, Lindblom 1990). Others factors that might contribute to cost are cognitive-e.g., attentional and memory-limitations or time (e.g. Neumann et al. 2014).

As has been pointed out (e.g., Pouplier 2012), some of these notions of cost are difficult to directly measure. This is not an in-principle limitation though: rather it presents a methodological challenge. For example, there is a rich tradition of research on attentional and memory limitation in various domains of the cognitive sciences. One example directly relevant to phonology is research on the amount of subcategorical information listeners can maintain about the speech signal (and for how long, e.g., Bicknell et al. 2016; Connine et al. 1991; Crowder 1982; Szostak \& Pitt 2013; for review, see also Dahan 2010). Similarly, metabolic 
energy consumption — while difficult to measure — can be measured (e.g., Hoyt \& Taylor 1981, cited in Lindblom 1983 and discussed in Pouplier 2012).

Here we have little to contribute to the understanding of 'cost', other than to re-iterate that models of cost can be and have been developed (see, e.g., Hayes 1999 for a model of articulatory difficulty of voicing for different places of articulation, and an account in terms of optimality theory that draws on this model). These models provide quantitative measures of costs, thus lending themselves to quantitative evaluation, including in the context of MOP. Finally, we note that even while no single objectively quantifiable measure of cost is available, it is still often possible to make predictions about the relative cost for closely related structures within a language (e.g., based on articulatory biases/error rates in production). We return to this point in Section 4, where we illustrate how predictions can be derived from the proposed framework. Here we briefly describe some intuitive cases of the redundancy-cost trade-off.

The general trade-off between increasing signal specificity (by adding redundancy) and reducing resource cost is reflected in the empirical findings summarized in the previous section: these findings suggest that signal specificity is increased only when message predictability alone would not be expected to lead to sufficiently accurate message transmission. This tendency to conserve cost when message predictability is high is perhaps most striking in deletion and omission phenomena. For example, in casual speech syllable-final plosives are often elided (e.g., t/d deletion, Raymond et al. 2006). This tendency is increased for contextually predictable words (e.g., Gahl \& Garnsey 2004; Raymond et al. 2006). Similarly, contraction of auxiliaries or negation in conversational English is more common when the meaning conveyed by those morphemes is contextually predictable (e.g., she's vs. she is, Frank \& Jaeger 2008; see also Bybee \& Scheibman 1999). ${ }^{13}$ The same general tendency has been observed with regard to the

\footnotetext{
${ }^{13}$ In practice, researchers need to estimate contextual predictability-i.e., the conditional probability of the unit in question given the context, $\mathrm{p}$ (unit $\mid$ context) (though some studies have used the joint frequency or mutual information instead, which is not the same as predictability, e.g., Bybee \& Scheibman 1999). A number of different methods are used for this purpose, including corpus-based $n$ grams capturing the contextual predictability given the preceding or following lexical context (e.g., Aylett \& Turk 2004; Bell et al., 2003; Raymond et al. 2006; Frank \& Jaeger 2008), subcategorization probabilities (e.g., Gahl \& Garnsey 2004), or more complex probabilistic models (e.g., probabilistic phrase structure grammars, Levy \& Jaeger 2007), or cloze estimates, based on continuations in context completions (e.g., Tily \& Piantadosi 2009; Kravtchenko 2015). For reasons that go beyond the scope of this paper (but see, e.g., Smith \& Levy 2013), many researchers have used the estimated surprisal or Shannon information in context-i.e., the log-transform of the reciprocal of the estimated contextual probability-rather than the raw untransformed contextual predictability estimate (e.g., Bell et al. 2003, 2009; Frank \& Jaeger 2008; Jaeger 2010, 2011;
} 
omission or insertion of optional morphology (Kurumada \& Jaeger 2015), truncation (Mahowald et al. 2012; Shaw et al. 2014), function words (e.g., Jaeger 2010, 2011; Wasow et al. 2011a,b), and arguments (Kravtchenko 2015; see also Resnik 1996). In all cases, the cost of additional material — increasing signal specificity and redundancy — is more likely to be invested when message predictability is low (for review of these and related findings, see Jaeger \& Buz, in press).

The general interplay between robustness and resource cost that we assume is summarized in (7) (based on Shannon 1948).

\section{(7) Biases toward Effective Message Transmission}

a. All else being equal, increasing signal redundancy and hence signal specificity, increases system robustness, but incurs greater resource cost.

b. All else being equal, reducing signal redundancy reduces resource cost, but can increase transmission error through lower signal specificity.

Putting everything together discussed so far, Figure 4 provides a visual representation of the complete trade-off relations between message transmission accuracy and resource cost (see also Cohen Priva 2012).

Kravtchenko 2015; Levy \& Jaeger 2007; Pluymaekers et al 2005; Tily \& Piantadosi 2009; Tily et al. 2009). 


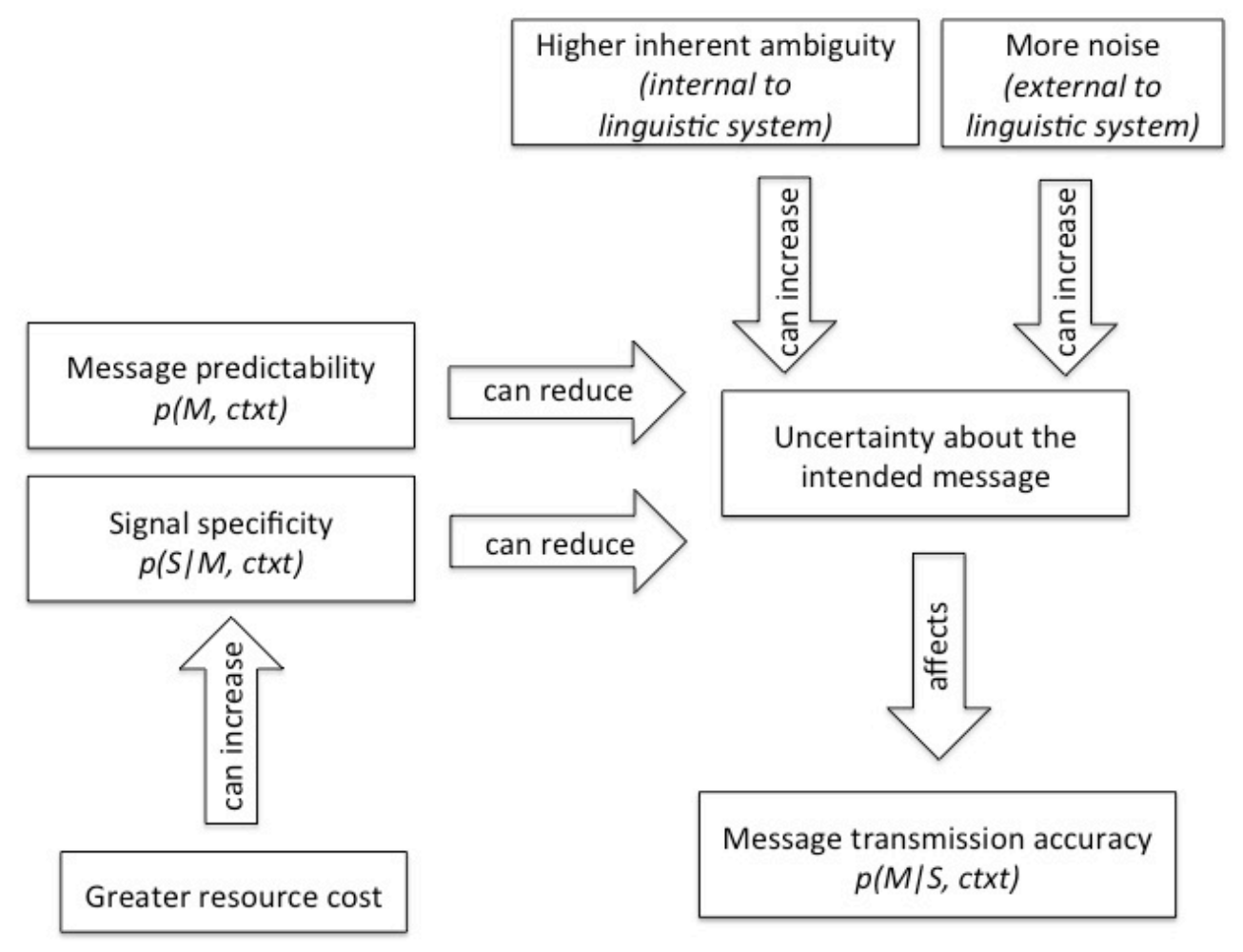

Figure 4: The trade-off between message transmission accuracy and resource cost

There is now also evidence that the cumulative effect of this trade-off between redundancy and cost comes to be reflected in the lexicon over time (e.g., Cohen Priva 2015; Kanwaal, in prep; Piantadosi et al. 2011, 2012). For example, Cohen Priva (2015) presents evidence that obstruent duration and deletion patterns are better described in terms of the average obstruent predictibility in phonological context. ${ }^{14}$ Similarly, the typical durations of words seems to be a function of their average predictability (Seyfarth 2014). Likewise, the finding that phonetic cues distinguishing a word from a lexical minimal pair tend to be hyperarticulated even when the minimal pair competitor is not salient in the context (Baese-Berk \& Goldrick 2009; Nelson \& Wedel submitted; Wedel et al. in prep), might be the cumulative phonologized effect of

\footnotetext{
${ }^{14}$ The average contextual predictability can be estimated by taking a large corpus (with many different context, e.g., lexical contexts, as would be captured by an $n$ gram model). The average predictability is the sum of all context-specific predictabilities weighted by the probability of the context,

$$
-\sum_{c} P(C=c \mid W=w) \log P(W=w \mid C=c)
$$

(see, e.g., Cohen Priva 2015; Piantadosi et al. 2011, who use the average in context Shannon information, also sometimes called informativity, e.g., Cohen Priva 2008; Seyfarth 2014). For discussion of different measures, see also Cohen Priva (2015) and Jaeger (2006).
} 
hyperarticulation in the presence of contextually available competitors (as proposed in Buz \& Jaeger, in prep). Finally, the number of segments in a word's phonological citation form correlates with its average predictability across contexts (Manin 2006; Piantadosi et al. 2011).

In Section 4 below, we illustrate and discuss this for the specific case of strong/weak phonological patterns. In continuing, we make the common simplifying assumption that increasing signal specificity tends to correlate with increasing cost (even though some phonetic cues may come for "free"; see, e.g., Stevens \& Keyser 1989). Therefore, if linguistic systems evolve under a bias toward message transmission accuracy over contexts with respect to resource cost, we expect that grammaticalized phonological patterns will reflect this trade-off (e.g., Lindblom 1990). Indeed, as we propose below, viewed from this perspective we are able to explain several otherwise arbitrary properties of phonological systems and phonological change.

\section{A Message-oriented Phonological Approach to Strong and Weak Patterns}

In this section we propose that a motivation for strong and weak phonological patterns and their distribution cannot be understood without reference to the trade-off between message transmission accuracy and resource cost. More concretely, when a PUC (phonological-unit-incontext) contributes more to average message transmission accuracy relative to its resource costs, the prototypical pronunciation of that PUC should evolve toward a stronger, more redundant form. Conversely, when a PUC contributes little to average message transmission accuracy relative to its cost, a weaker, less redundant variant should become prototypical in that context. ${ }^{15}$

A major conclusion of the MOP framework is that the transmission of meaning-bearing units such as words plays a causally central role in the development of phonological patterns over time. In support of this, we will present evidence in the following sections suggesting that a full account of a language's phonology requires reference to the lexicon and its context of use.

\footnotetext{
${ }^{15}$ For the present purpose, we leave open the specific mechanism(s) by which strong or weak variants arise (though see Section 5.2, where we return to this point). Every sound category is characterized by distributions of variants at the individual and community levels, shaped in part by available articulatory, perceptual, grammatical and social pathways for the creation of variation (see, e.g., Ohala 1989, Labov 1994, Blevins 2004). For example, it is both possible that informationally more effective variants are selected out of a pool of existing variation, and/or that a bias toward effective message transmission can itself contribute to the creation of informationally-effective variants (see references in footnote 10). Under either scenario, a bias toward message transmission effectiveness is predicted to interact with the range of synchronic and diachronic factors previously identified in the literature to influence phonological patterning in a given language.
} 
Conversely, we will show in Section 4.4 below that when we attempt an alternative, soundcentric account, we make precisely the wrong predictions for phonology.

\subsection{Phonology and 'the right sort of redundancy'}

As noted above, 'resource cost' is grounded in a heterogeneous set of phenomena and we do not yet have access to a single objectively quantifiable measure of cost. We can nonetheless draw inferences about the larger system by comparing patterns in which the cost relationships are likely to be similar, such as for patterns involving the same class of sounds and contexts. We can generate testable hypotheses by comparing patterns with presumed similar costs, even if we cannot precisely measure cost itself. In doing so, we will use the concept of 'change in uncertainty per unit cost' $\left(\Delta \mathrm{H} / \operatorname{Cost}_{\mathrm{x}}\right)$ to describe the average reduction in uncertainty in messages through making a particular change of $\operatorname{cost} x$ to signals; the symbol H (Eta) corresponds to entropy, the information theoretic measure of uncertainty (Shannon 1948). Each PUC in a signal has the potential to contribute some degree of uncertainty reduction to the process of message transmission. Relativized to a given cost, we can reason about what changes to PUCs will result in a larger average decrease in uncertainty in message transmission, and conversely, what changes will have less effect on uncertainty.

As stated in (8), our approach makes two predictions about which changes to the phonetic properties of PUCs within a message are more likely to be observed.

\section{(8) Resource cost and Uncertainty Reduction}

a. For a given increase in cost, we expect changes in PUCs that on average better reduce uncertainty about the message.

b. For a given degree of uncertainty reduction about the message, we expect changes to the phonetic properties of PUCs that on average better reduce cost.

This set of predictions parallels and elaborates on Pierce's quote at the beginning of Section 3 in that language change involves a continual process of accreting the 'right sort of redundancy' while eroding away 'inefficient redundancy'.

Figure 5 displays in graphical form how these elements fit together. The area in the figure can be thought of an abstract space that includes the potential sound patterns associated with a given 
message (e.g., a word), where the question that we are attempting to answer is, for any given PUC in that message, how much resource investment would be rational? This variable degree of resource investment is shown along the y-axis. Recall from Section 3.3 that this is essentially the question of how much redundancy should be added or removed in order to increase or decrease signal specificity. PUCs that require a larger investment in resources are situated toward the top of the figure while those that do not are located toward the bottom.

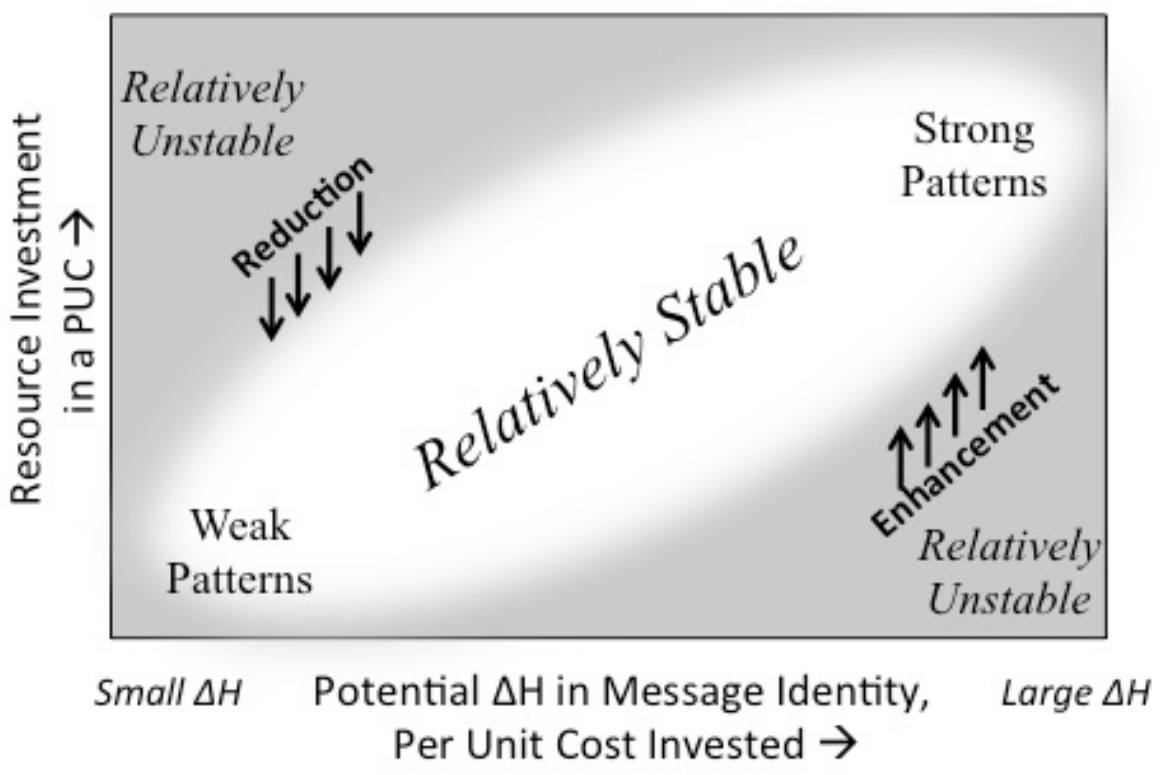

Figure 5: Phonological patterns as emerging from the effective trade-off between accurate message transmission and resource cost

The variable degree of change in the uncertainty for a given investment of resources $\left(\Delta \mathrm{H} / \operatorname{Cost}_{\mathrm{x}}\right)$ is shown along the $\mathrm{x}$-axis. This degree can, as we have discussed in Section 3.4, be influenced by message predictability or signal specificity, i.e. the degree to which a signal is close to being unique to a particular message. ${ }^{16}$ PUCs that have the potential to make a small contribution to reducing uncertainty occur to the left while those that could make a large contribution are toward the right.

\footnotetext{
${ }^{16}$ In the following discussion, we are assuming — for simplicity's sake - that all messages have on average the same communicative utility (i.e., are equally important or relevant to the speakers' communicative goals). This is most likely a wrong assumption (though with regard to its average consequence for understanding phonological systems, rather than individual situations, it might be sufficiently close to the truth), but does not affect the general logic outlined here (the notion of communicative utility could easily be integrated into the framework proposed here).
} 
Figure 5 also illustrates regions where a PUC is predicted to be diachronically stable or unstable. The highlighted space along the positive diagonal and is relatively stable by having, on average, an effective trade-off of message transmission accuracy and resource cost. The upperleft and lower-right quadrants are regions in which PUCs are predicted to be unstable and susceptible to increases or decreases in signal redundancy. We illustrate in more concrete terms in Section 4.2.1 below. Assumed in this approach is the view that diverse causes and pathways for sound change are always present, at multiple levels of description (e.g., Blevins 2004). Through focusing on PUC stability within the abstract space defined in Figure 5, we can sidestep the issues both of pathway, and of actuation versus propagation of sound change: PUCs that contribute cost-effectively to message transmission accuracy are more likely to persist, however they arise (see discussion in Soskuthy 2015). For example, if some diachronic chain of events unrelated to a bias toward effective message transmission nonetheless results in an effective phonological pattern, that pattern is predicted to be stable (for a plausible example, see Hansson 2003).

In the remainder of this section, we show how the proposed framework accounts for several properties of weak and strong patterns, including the ones discussed in Section 2. In particular, we focus on five factors, listed in Figure 6, that serve to illustrate the logic of our approach. The first two factors are properties of the (meaning-bearing) message: the predictability of a word and its syntactic class. The fifth factor, lexical competition, also refers to a property of the messagespecifically, the existence of other messages with similar phonology that therefore increase the intended message's inherent ambiguity. These three factors are examples of properties that the present approach predicts to affect the distribution of weak vs. strong patterns; sound-centric approaches to phonology make no such prediction (see Section 4.4).

The remaining two factors describe properties that affect the bottom-up perception of PUCs, including the advantages associated with the relative position of a segment in a word, as well as its local phonetic context. Like traditional models of phonology, our approach takes into account the possible influence of such factors. There is, however, a critical difference between soundcentric approaches and our proposal. In the present approach, the effect of these factors on the weakening or strengthening of PUCs is predicted to be mediated by the degree to which a PUC is able to reduce message uncertainty given its resource cost. 


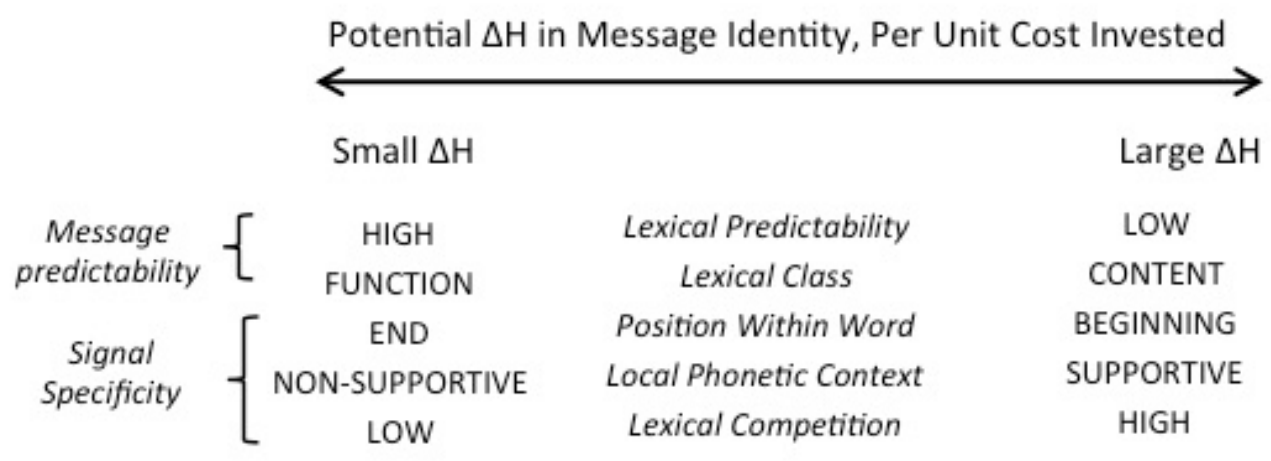

Figure 6: Some factors and their potential effect on a change in the uncertainty of a message given resource cost

Crucially, what we propose is that change in uncertainty is a unifying concept that helps us understand the interplay of the diverse set of factors in Figure 6 that have each independently been shown to affect phonological patterns, as described with regards to the weak and strong patterns in Section 2. That is, an investment of resources in a particular signal will have a smaller or larger impact on accurate message transmission, depending on the specific values of each of these factors, indicated on either side of the properties in Figure 6. For example, high/low lexical predictability will, all else being equal, be associated with a smaller/greater reduction in message uncertainty for a given investment of resources. We discuss each of the properties in turn below. It is important to stress that these are not random properties; they all play a role in successful message transmission, though they can of course be at odds with each other in any individual example. By laying out what these factors are and how they align with the larger unifying bias toward accurate message transmission and resource cost-effectiveness, we hope to forge a path for future research: individual factors can be quantified in particular situations, and their interaction studied to determine how they play out in shaping phonological systems.

In the following section, we focus on better understanding the horizontal axis in Figure 5 by describing the five factors noted above associated with weak and strong sound patterns.

\subsection{Message Predictability}

We focus in this section on lexical properties relating to message predictability that can give rise to strong and weak patterns. We begin with predictions for lexical predictability and then turn to 
effects related to lexical class; as noted above, we suggest that both are derivative of the more fundamental property of message predictability.

\subsubsection{Expected surface forms and lexical predictability}

When the message in context has high predictability (i.e., $\mathrm{p}(\mathrm{M} \mid \mathrm{ctxt})$ is high), increasing signal specificity is likely to have a smaller effect on reducing the uncertainty about the message's identity. ${ }^{17}$ For such high-predictability messages, our framework predicts a tendency for speakers to produce relatively reduced variants of PUCs, and that over time, typically high-predictability messages will tend to develop prototypical surface forms that are more reduced than the phonologically expected form (cf., Bybee 2001; Pierrehumbert 2002; see also Piantadosi et al. 2011; Seyfarth 2014).

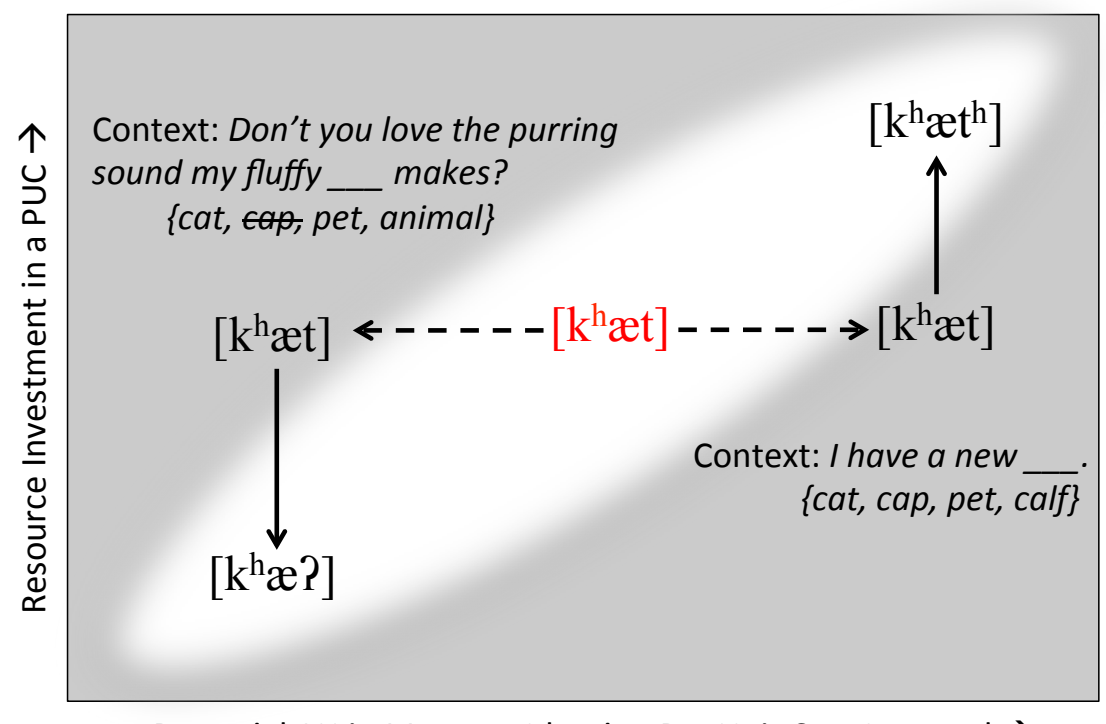

Potential $\Delta H$ in Message Identity, Per Unit Cost Invested $\rightarrow$

Figure 7: Example of how a particular surface form may vary in different contexts to maintain an effective trade-off between accurate message transmission and resource cost.

\footnotetext{
${ }^{17}$ In particular, if speakers aim to achieve some target error rate (e.g., which itself can be understood as balancing the resources invested into increasing the probability of being understood on the first attempt against the social and cognitive resource costs associated with having to repair miscommunication), this corresponds to a target uncertainty (e.g., in bits) they are willing to accept on the comprehender's side. Thus, if a particular message transmission is already expected to result in less uncertainty than the targeted uncertainty threshold, investing into it further increases in signal specificity is not efficient.
} 
This process is illustrated graphically in Figure 7. Start from the assumption that the message to be transmitted is the word cat, and that its phonologically expected form in some language is [k $\left.\mathrm{k}^{\mathrm{h}} \mathfrak{} \mathrm{t}\right]$. This is the form shown in the center of Figure 7; we assume that this form has achieved stability for the language up to this point. We can then focus on a particular PUC in this word such as the final segment, [t], and assume that it has an average cost, and contributes an average amount to message uncertainty reduction across all instances of the word. In any particular instance in which the word is uttered, however, one must take into account a variety of other factors. For example, if the word is being uttered in the context of the sentence "Don't you just love the purring noise my fluffy ___ makes?”, the word will be highly predictable (i.e., many similar competitors, such as cap, are not plausible). None of the PUCs would contribute much to uncertainty reduction because of this general predictability. The final $[\mathrm{t}]$ in particular will have an especially low contribution because of other factors we will discuss just below, such as the fact that it is at the end of the word (§4.3.1) and the lexical competitors in this context (e.g., animal, pet) are not close phonologically (§4.3.3). Thus, the normally stable pronunciation of this word in this specific context will be positioned to the left in the figure - the production of [t] is the same in both cases, and so involves the same resource cost, but is less worthwhile in terms of accurately transmitting the message. As a consequence, the MOP framework predicts that speakers will be biased toward lower-cost variants of the PUC relative to the phonologically expected form in this message, even though those variants might have (slightly) lower signal specificity and thus increase uncertainty. Thus, if the word cat is produced often in such high predictability contexts, the MOP framework predicts that its prototypical pronunciation may continue to reduce, say, toward $\left[\mathrm{k}^{\mathrm{h}} æ\right.$ ? $]$. In this form, the coronal constriction of the final [t] is not needed for accurate message transmission; that is, it is redundant (see (5)).

On the other hand, the same message, with its phonologically expected form $\left[\mathrm{k}^{\mathrm{h}} \mathfrak{t}\right]$, might be produced in a context such as "I have a new __," in which the intended message has low predictability, and in particular the competitors might include words that differ in the coda. In this case, the form $\left[\mathrm{k}^{\mathrm{h}} æ \mathrm{t}\right]$ will be positioned to the right in the figure, that is, investing the same amount of resources to produce the usual $[\mathrm{t}]$ is possible, but investing more would have a greater impact on reducing uncertainty about which word is intended. ${ }^{18}$

\footnotetext{
${ }^{18}$ This general unpredictability might be compounded by having lexical competitors with similar signals that are about equally predictable in this context (see 4.3.3 below).
} 
Both of these changes, to $\left[\mathrm{k}^{\mathrm{h}} \mathfrak{x}\right.$ ] in a high predictability context or to $\left[\mathrm{k}^{\mathrm{h}} \mathfrak{x} \mathrm{t}^{\mathrm{h}}\right]$ in a low predictability context, might simply occur as one-time pronunciation variants based on the context. On the other hand, if such contexts arise frequently across words with similar possible PUCs, then we have the possibility of phonological change. For example, if words ending in [ $\mathrm{t}$ ] in this language are produced frequently and in predictable contexts, glottalization as a phonological process may arise, and the new phonologically expected form of this word might be $\left[\mathrm{k}^{\mathrm{h}} \mathfrak{} \mathfrak{}\right.$ ?, and simply exist as a relatively stable form in the lower left-hand corner.

\subsubsection{Weak and strong patterns by lexical class}

The observation that typologically, lexical categories typically behave as strong contexts while functional categories tend to be weak also relates, we suggest, to message predictability. As noted in Section 2.2, for example, material in roots and stems tends to be preferentially preserved over affixal material (see e.g. Urbanczyk 2011 for an overview of effects). It has been widely hypothesized that this preference stems from some communication-based bias to preserve less predictable lexical material over more predictable structural material (Beckman 1998; Blevins 2004; Krämer 2007; Ussishkin \& Wedel 2002). One such case noted earlier (see §2.2) concerns the cross-linguistic patterning of sequences of two vowels $\left(V_{1} V_{2}\right)($ Casali 1997, 2011). When the vowel sequence results from the concatenation of a prefix and stem, the typologically common pattern is for $\mathrm{V}_{1}$, i.e. the prefix vowel, to delete. Conversely, $\mathrm{V}_{2}$ deletion typically occurs when $V_{1}$ is part of a root and $V_{2}$ is part of a suffix or other function word.

These patterns are consistent with predictions of the current framework: investment in PUCs in functional morphemes tends to lead to a smaller reduction in uncertainty because function morphemes are highly frequent and predictable in the local morpho-syntactic context — that is, they are classes of words that tend to exhibit the kind of high predictability described in $\S 4.2 .1$. As a consequence, reducing cost in function material should on average have less impact on reducing uncertainty in message transmission relative to the same reduction in a content morpheme (for discussion, see Ussishkin \& Wedel 2002).

To illustrate further, assume a /CV-/ functional element prefixed to a vowel-initial stem, and assume that maintaining a clear signal for both the affix-final and stem-initial vowels requires greater cost than reducing one of the two vowels. If the form with both vowels intact is located above the diagonal in Figure 5 and thus relatively unstable, the degree of uncertainty reduction 
provided by the affix vowel is low relative to the cost of maintaining that vowel. Given this, we predict that speakers will be biased toward production of reduced variants of that affix vowel. Over time, the prototypical pronunciation of this affix in vowel-initial stem context may shift to complete loss of the vowel. Phonological patterns that refer specifically to word classes such as function words are typologically common, so if gradient vowel reduction in function words in this context occurs predictably enough, we expect that this pattern may be phonologized in the language.

\subsection{Signal Specificity}

Recall that Bayes theorem (E1) predicts message transmission accuracy to be a function of the product of message predictability and signal specificity, i.e. the degree to which a signal is consistent with a particular message as opposed to all others. In this section we argue that the reason weak and strong patterns tend to be associated with word position, local phonetic context, and degree of competition from words with perceptually similar signals (henceforth, lexical competition) is because all influence the signal specificity of the message.

\subsubsection{Weak and strong patterns by word position}

As discussed in Section 2.1, beginnings of words generally behave as strong contexts crosslinguistically while word endings are typically weak (Beckman 2001; Smith 2004). Thus, investing resources at the ends of words will tend to have a smaller impact on reducing uncertainty than the equivalent investment at the beginnings of words. As examples, recall the strong pattern of word-initial fortition in Luganda and the weak pattern of word-final degemination in Tiberian Hebrew (see §2). Similarly, non-alternating distributional patterns typically reveal more contrasts word-initially than word-finally, as found in Leti where initial geminates and consonant clusters are supported only word-initially, and Turkish where midround vowels are limited to word-initial syllables in native vocabulary. Our framework straightforwardly predicts this typological distribution as a consequence of the observation that listeners process spoken messages incrementally below the level of the word.

Listeners begin matching cues in the signal to the lexicon as soon as they have evidence for a possible word initiation-point in the speech stream (e.g., Cutler \& Norris 1988, Saffran, Newport \& Aslin 1996, McQueen 1998), as has been supported experimentally with methods such as 
gating (Zwitserlood 1989) and eye-tracking (Allopenna et al. 1998; see also Magnuson et al. 2007, McMurray, Tanenhaus \& Aslin 2002). Evidence suggests that as the signal unfolds in time, listeners continually update a set of probable lexical alternatives, with the result that the number of probable alternatives tends to decrease as the signal is processed (for a review, see Dahan \& Magnuson 2006). ${ }^{19}$

As a consequence of continuous updating, the possible information contributed by a given phonological category is limited by the reduction in uncertainty that has already been provided by cues earlier in the signal (van Son \& Pols 2003) ${ }^{20}$ As an example, consider a signal for the word madam (/mædəm/). Cues consistent with the initial $/ \mathrm{m} /$ provide a potentially large reduction in uncertainty by reducing the set of contextually-possible alternatives to those words that begin with $/ \mathrm{m} /$. Cues to the word-final $/ \mathrm{m} /$ contribute less, because now in addition to whatever information the listener has from the wider context about the probability of the word madam, prior processing of cues within the signal has additionally constrained the set of lexical alternatives. On average then, a given phonetic cue tends to reduce uncertainty about word identity less when near the end of a word than when near the beginning (see Figure 8).

The MOP framework predicts that given incremental processing of the signal, all else being equal, speakers should be biased toward production of enhanced/more redundant variants of PUCs earlier in a word, and reduced variants later in the word. In support of this, in an investigation of segmental information and hyperarticulation in a corpus of spoken Dutch, van Son \& Pols (2003) showed that segments are relatively hyperarticulated in relation to their contribution to identifying a word relative to competitors (see also van Son \& van Santen 2005). Segmental information to word identity was measured as a function of the segment's position within the word, taking into account the predictability of the target word given the context, including the prior segmental information. They found that segments at the beginning of the word tended to be articulated more fully, consistent with the fact that on average, the closer a segment is to the beginning of the word, the more disambiguating information it contributes, as measured in this way (Figure 8).

\footnotetext{
${ }^{19}$ Lexical alternatives that do not match the segments processed so far do not seem to be categorically ruled out (e.g., Allopenna et al. 1998). Rather listeners seem to maintain a certain degree of uncertainty about the input processed so far (for review, see Dahan 2010) — which would indeed be a rational response to the noisiness of the percepts (Bicknell, Jaeger, \& Tanenhaus, 2016, submitted).

${ }^{20}$ Note that this argument, as presented in van Son and Pols (2003), makes a simplifying assumption that the word onset has been accurately detected from the signal as processing proceeds.
} 


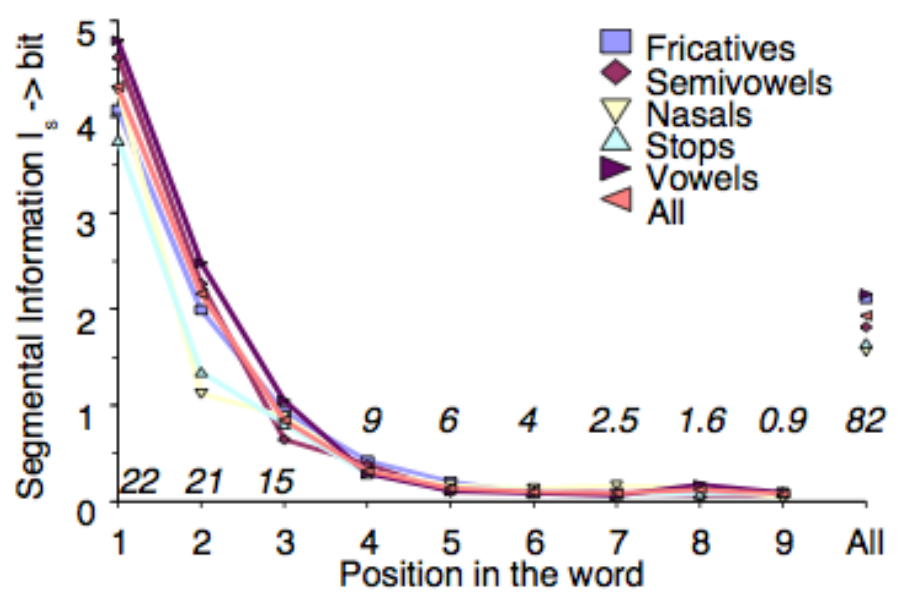

Figure 8. Relation between average segmental information $\left(I_{S}\right)$ and the position in the word grouped by manner of articulation in a sample of Dutch. The number of segments (x1000) for each position is indicated with italic numbers (van Son \& Pols 2003).

Thus, if a given PUC is persistently enhanced when it occurs toward the beginning of words, or persistently reduced toward the end, this pattern may become phonologized over time. Via this general pathway, incremental processing of the speech signal should lead to the typologically common tendency for strong patterns to cluster near word beginnings and weak patterns at word ends.

\subsubsection{Weak and strong patterns by local phonetic context}

In sub-lexical domains, descriptions of typologically common strong and weak positions generally make reference to prosodic structure (e.g. onset/coda, stressed/unstressed) or to segmental structure (e.g. prevocalic/preconsonantal). Examples noted in Section 2.3 include onset strengthening of glides to fricatives in Porteño Spanish (Lozano 1979), coda neutralization of phonation contrasts in Korean (Cho 1990), and the full/reduced vowel inventories in English stressed/unstressed syllables. All else being equal, a phonological unit that occurs in a local phonetic context that supports greater cue perceptibility will make a larger contribution to reduction of uncertainty in the transmission of messages than the same phonological unit in a context that does less to support its cues. As a consequence, the MOP framework predicts that higher redundancy will often be more cost-effective for accurate message transmission in prosodic/segmental contexts in which cues are more perceptible. We thus expect higher resource 
investment and thus higher redundancy to develop in these contexts (see also Cohen Priva 2012). ${ }^{21}$

We illustrate with a typologically common pattern involving intervocalic consonant clusters, $\mathrm{C}_{1} \mathrm{C}_{2}$ (e.g. brow $[n][b] a g$ ), as noted in Section 2.3: typically, $\mathrm{C}_{1}$ shows a weak pattern, while $\mathrm{C}_{2}$ shows a strong pattern. The weak status of $\mathrm{C}_{1}$ is associated with the common observation that consonants in these positions typically have weak perceptual cues, as opposed to consonants in $\mathrm{C}_{2}$ position (e.g. Steriade 1995, 2001; Flemming 1995; Jun 1995; Padgett 1995; Boersma 1998; Hume 1999; Côté 2000; Blevins 2003; Seo 2003). For example, plosives in $\mathrm{C}_{2}$ position have an acoustic cue package that includes vowel formant transitions into the following vowel as well as a release burst, which is often lacking in $\mathrm{C}_{1}$ plosives. Because of these strong cues, PUCs in $\mathrm{C}_{2}$ position are likely to be highly perceptible. Consequently, viewed in terms of the current approach, this makes $\mathrm{C}_{2}$ a good position to invest articulatory resources in, since it will contribute more to specificity of the signal and in doing so, reduce uncertainty about the intended message. Conversely, since consonants in $\mathrm{C}_{1}$ position are generally produced with weaker phonetic cues, increasing redundancy (and cost) is only beneficial if there is a particularly large pay-off in uncertainty reduction, such as if there are many lexical competitors distinguished at this position. See Section 4.3.3 for related discussion and examples.

A similar account extends to strong/weak positions linked to prosodically prominent/nonprominent contexts. Stressed syllables, for example, provide a better investment in resources for hosting phonological units that contribute more to communicating a message. The reason, as above, relates to acoustic differences between stressed and unstressed syllables: cues in stressed syllables are more highly perceptible than those in unstressed syllables (e.g., Cole, Jakimik \& Cooper 1978).

Codas are often targets of reduction relative to onsets, which, as noted above, has been argued to arise from lower general perceptibility for many cue-types in coda contexts, e.g., in pre-obstruent or word-final position (e.g., Steriade 2001, 2008). The approach we argue for here predicts that incremental processing (discussed in Section 4.3.1 above) provides an additional,

\footnotetext{
${ }^{21}$ Note that traditional phonological approaches account for such effects by reference only to the local phonetic context; as we show in Section 4.4, there is evidence that this sound-centric view does not entirely make the correct predictions and instead, higher levels of meaning-bearing units need to be referenced as well.
} 
independent cause for onsets to behave as stronger contexts relative to codas. This is because averaged over a lexicon, onsets are necessarily closer to the beginning of words than codas. Monosyllabic words, for which syllable onsets are word-initial and codas are word-final, provide an extreme (and highly frequent) example. Provided that word boundaries are successfully identified in processing, syllable onsets then tend to provide more disambiguating information

during lexical access than codas, simply because they on average are closer to the beginning of the word (see Figure 5 above). On this basis, the observation that onsets tend to be enhanced while codas reduced and that correspondingly, onset inventories tend to be larger than coda inventories, may be at least in part an epiphenomenon of their different average word positions. This is consistent with the prediction that laryngeal feature neutralization may often begin diachronically in word-final context, generalizing later to syllable- or morpheme-final context (Blevins 2006). One open question for future research is to identify data that decorrelates prosodic prominence from average word position to explore these two potential sources of the onset advantage.

\subsubsection{Weak and strong patterns by lexical competition}

As reviewed in Section 3.5 above, a growing body of evidence indicates that speakers modulate phonetic detail in individual utterances in a way that increases phonetic distinctiveness relative to lexical competitors that are plausible in the context (Baese-Berk \& Goldrick 2009; Kirov \& Wilson 2011; Buz et al 2016, in prep; Seyfarth et al. 2016). Further, evidence suggests that utterance level effects compound over time, shaping long-term lexical representations. What are our predictions for phonological pattern formation given evidence that redundancy in phonetic cues within individual words is modulated to maintain perceptual distance to specific lexical competitors? As a general proposition, we predict that in cases where there is a lower degree of lexical competition - and therefore, a high degree of signal specificity already - that an investment to enhance PUCs will have a smaller impact on reducing uncertainty about the identity of the message than in cases where the degree of lexical competition is higher.

On the basis of similar information-theoretic notions as those underlying this paper, many linguists over the last century have predicted that phoneme contrasts that do more to support communication should be less prone to loss through merger (see e.g., Zipf 1932, Trubetzkoy 1939, Martinet 1952, Hockett 1955, Lindblom 1990, Nettle 1998, Surendran \& Niyogi 2006). 
Wedel et al. (2013a,b) recently provided statistical evidence for this proposition by showing that in a diverse set of languages, the larger the number of minimal pairs that are distinguished by a given phoneme contrast, the more likely that contrast is to be preserved over diachronic time; conversely, a smaller number of minimal pairs is associated with a higher probability of loss of the contrast through merger.

A similar relationship with lexical contrast is also found in patterns of vowel duration contrast in Te Reo Māori. Historically, the language included a length contrast in the system of monophthongs: [i]-[i:], [e]-[e:], [a]-[a:], [u]-[u:], [o]-[o:]. However, the distinction between short and long vowel pairs has decreased in all cases except [a]-[a:] (King et al. 2010). As predicted, this lack of reduction can be attributed to specific properties of the language's lexicon: Todd (2012) found the number of lexical competitors distinguished by the [a]-[a:] contrast to be significantly greater than for any of the other pairs.

As a final example, the low functional load of a lexical contrast is shown to promote tone merger in Hong Kong Cantonese (HKC). In HKC's system of six lexical tones, two pairs of tones are merging: T2-T5 and T3-T5 (Kei et al. 2002; Bauer et al. 2003; Mok \& Wong 2010a; Mok \& Wong 2010b; Wong 2008). While the tones in each pair are acoustically similar, Tsui (2012) shows that acoustic similarity alone cannot explain the merger as there are other phonetically similar pairs in the language. However, the tone pairs T2-T5 and T3-T5 also distinguish few words. Tsui (2011) concludes that both functional load of these categories-i.e., the average contextual distinguishability of the words distinguished by the phonological categories - and their perceptual similarity are necessary conditions for predicting the Cantonese tonal merger data (see Burchill \& Jaeger 2016 for a model that begins to incorporate both of these aspects).

The MOP framework is compatible with these findings. As before, we start from the assumption that the phonologically expected surface form of a PUC in all words shifts over time under the influence of biases on the form of PUCs across individual instances of messages. If a PUC is frequently enhanced to support signal specificity, increasing the distinguishability of the lexical item it is part of from other lexical items, then the prototypical, phonologically expected surface form of that PUC is likely to be enhanced, or at least resistant to reduction. Conversely, if a PUC contributes little to signal specificity or contributes to the signal specificity of only a 
few lexical items, the PUC is likely to undergo reduction or at least be resistant to enhancement. This would then set the stage for eventual merger or deletion.

With all of this in mind, we can also make predictions about the joint effects of message predictability and signal specificity. As mentioned above (footnote 18), MOP predicts that contextually probable lexical competitors should magnify the effects of competitors described so far. The effect of lexical competitors with similar signals on the reduction (or lack of enhancement) of a PUC should be exaggerated further if its lexical competitors tend to be improbable in the contexts in which the target message occurs (and mutatis mutandis when all lexical competitors tend to be particularly improbable in the contexts in which the target message occurs).

Finally, we note that any weakening of a PUC could occur globally across all contexts, or be limited to neutralization in specific contexts (for an example of this trajectory of weakening to neutralization, see the discussion of intervocalic $\mathrm{t} / \mathrm{d}$ weakening in English in Section 4.5.1 below). The latter outcome-neutralization in specific contexts - is perhaps most likely when there is a sufficiently larger number of contexts in which a PUC contributes little to uncertainty reduction, while there are other contexts in which the PUC makes critical contributions to uncertainty reduction. For data that are compatible with this prediction, along with related discussion, see Blevins \& Wedel (2009) and Wedel, Jackson, \& Kaplan (2013).

\subsection{Revisiting the message: sub-lexical or higher?}

MOP focuses on balancing robustness and cost at the level of meaning-bearing units, i.e. morphemes, words and higher levels of meaning. What would happen if we instead assessed the trade-off between robustness versus resource cost exclusively at a more traditionally phonological level, such as the level of the phoneme? The Bayes' Rule formulation of message transmission in (E1) does not stipulate the analytical level(s) at which we define message, context and signal, so we could take the message (M) to be a phoneme, and the context as the local phonological context, the word and broader context that the phoneme occurs in. In this case, the signal $S$ would be the set of phonetic cues that map to the phoneme $M$ in context. Just as above, the probability of $M$ would be dependent on factors such as the set of competitor phonemes given the phonotactic context, as well as the larger discourse and sentence context that makes the phoneme's context more or less probable. Likewise, it would also be dependent on 
signal specificity: the degree to which the phonetic cues in the signal match the intended phoneme category relative to how well they match other phonotactically possible phoneme categories. In this framing, a bias toward accurate message transmission would act to support phoneme category identification in communication, rather than that of a meaning-bearing unit such as a lexical category. Given a bias toward accurate, yet cost-effective transmission of existing phoneme categories, we would predict compensatory enhancement in contexts in which phonetic cues to that phoneme category tend to be perceptually weak, and conversely we expect reduction in contexts in which cues to phoneme identity are easily perceptible. As examples, we would expect a cross-linguistic tendency toward enhancement of nasal place cues in preobstruent position where those cues are more difficult to perceive, and conversely, we would expect a cross-linguistic tendency for vowel place and rounding cues to be reduced in stressed syllables where those cues are more perceptible.

But this is precisely the opposite of what we do see: reduction tends to be associated with weak contexts and enhancement with strong contexts. If enhancement/reduction processes are focused on balancing robustness and cost of the transmission of phoneme categories themselves, we cannot easily explain this reversal. In weak contexts for example, transmission accuracy is already low, all else being equal, and further reduction of phonetic cues only makes the signal yet more difficult to perceive. As a consequence, the phoneme-as-message approach predicts that speakers should behave in the opposite way, expending more effort in disfavored contexts to maintain phoneme category transmission accuracy, not less.

In defense of the phoneme-as-message hypothesis, assumed in sound-centric approaches, one might argue that a predicted diachronic result of persistent reduction in these disfavored contexts is merger or deletion, resulting in a smaller inventory of possible phoneme contrasts, which in turn improves phoneme transmission accuracy despite a poor signal simply by decreasing the number of possible messages (i.e., phonotactically licit phoneme competitors). However, this does not rescue the phoneme-as-message hypothesis because in order to decrease the number of possible messages, the system has to pass through a state that the phoneme-as-message hypothesis predicts will be avoided: reducing phonetic cues for already difficult-to-perceive phonemes. Another problem for this hypothetical defense is that it predicts that phoneme inventories should steadily shrink to reduce ambiguity, unless some other bias is stipulated toward maintaining some arbitrary phoneme inventory size. The MOP approach, which 
emphasizes the goal of transmitting meaning-bearing messages, does not encounter this 'lookahead' problem: because there are typically many phonetic cues to a meaning-bearing unit, reduction of an inefficient phonetic cue in one part of the signal can be compensated by enhancement of another cue elsewhere. As a result, unlike the phoneme-as-message hypothesis, MOP correctly predicts the observed range of strong/weak patterns as well as trends in contextspecific inventories, such as larger vowel inventories in stressed syllables.

More broadly, MOP predicts that phoneme inventories are not maintained by an inherent bias toward maintaining some arbitrary number of categories, but rather indirectly by a bias toward accurate transmission of meaning (Wedel 2004, 2012). In this view, maintenance of structure within the signaling system of language is parasitic on the drive to communicate information about the structure of the external world (e.g., Wedel \& Fatkullin, submitted; Jaeger \& Ferreira, 2013, p. 32; McClelland, St. John, \& Taraban, 1989, p. 11; for related discussion of the role of representations in effective prediction, see also Kuperberg \& Jaeger 2016 and references therein). ${ }^{22}$

\subsection{Typologically uncommon strong and weak phonological patterns}

Earlier in this section we showed how MOP predicts common typological generalizations. Yet there are apparent exceptions to virtually all typological generalizations in phonology. Rather than framing this observation as a typical/exceptional dichotomy, we follow many previous contributions to the field and assume that less common patterns arise through less common combinations of causal factors or histories (for examples, see e.g., Coetzee 2002; Blevins 2004; Mielke 2008). As we will discuss below, such typologically less common patterns can often provide particularly useful tests of a theory's empirical predictions.

We have argued that phonological patterns in a language are influenced by syntagmatic and paradigmatic patterns in the uncertainty between signals for messages in that language. Specifically, PUCs that contribute more to reducing uncertainty in the process of message transmission are predicted to evolve stronger patterns than those that contribute less. For example, all else being equal, PUCs in word-initial, or phonetically prominent contexts are

${ }^{22}$ This is not to say that robust transfer of phonemes might not also play a role in shaping phonology. This is, in fact, quite compatible with the MOP framework since accurate phoneme transmission can be seen as contributing to the overall inference chain that underlies the successful communication of intentions (see also footnote 1). However, MOP predicts that the extent to which the accurate transmission of a phoneme matters in a given context depends on the information the phoneme carries about meaning-bearing units. 
predicted to contribute more to reducing uncertainty in message transmission, and thereby evolve strong patterns. However, as described in Section 4.2.3, a PUC's actual contribution depends on the degree to which it distinguishes contextually relevant message competitors in usage. As a consequence, MOP predicts that language-specific properties of the lexicon, including frequency and context of use, can create language-specific strong/weak patterns even though they may be typologically uncommon. If, in a given language, a PUC in a typologically strong position happens to on average distinguish few competitors-overall contributing weakly to reducing uncertainty in that language-MOP predicts that this PUC will tend to be reduced, even if it tends to be enhanced in most languages. Conversely, if a PUC in a typologically weak position happens to on average contribute strongly to reducing uncertainty because it distinguishes many competitors in usage, MOP predicts that the PUC will tend to be strengthened. The MOP framework suggests, then, that lexical and usage patterns should contribute to accounting for phonological patterns that may at first blush appear to violate typological generalizations. As such, typologically less common strong/weak patterns provide a useful test of the MOP framework. Next, we discuss several concrete examples of strong/weak patterns that fall into this category, beginning with a review of some types of unusual patterns.

In typologically weak positions where the expectation is for lower resource investment, i.e. lower redundancy, occasionally we find a language that shows a strong pattern instead. Such apparent counterexamples in positions expected to be weak may involve: (a) unexpected fortition or resistance to reduction/deletion; (b) a large inventory of contrasts; or (c) from a diachronic perspective, changes that prevent a loss of lexical contrast, i.e. phonemes that participate in shifts or splits (i.e., transphonologizations). Conversely, the following types of cases would be apparent counterexamples in crosslinguistically strong positions: (d) unexpected reduction or failure to enhance/strengthen; (e) a reduced inventory of contrasts; or (f) from a diachronic perspective, phoneme merger.

\subsubsection{Weak patterns in typologically strong positions}

Strong positions - such as onsets or word-initial positions-typically host strong patterns because PUCs there can contribute more to reducing uncertainty relative to resource cost. However, if a PUC contributes little to reducing uncertainty due to high contextual predictability or few existing lexical competitors, we expect it to be reduced even if it occurs in an otherwise 
typologically strong position. We illustrate with the example /t, $d /$ weakening in English. The pronunciation of the coronal stops as a flap intervocalically (e.g. [let] late vs. [lerə.] later) could be viewed as an apparent counterexample to the general typology of strong/weak patterns, given that intervocalic onset position is typically a strong context for voicing cues in stop consonants (Fujimura et al. 1978; Blumstein \& Stevens 1979; Wright 1996). Given that English has a t/d contrast one might expect it to be maintained in such a strong context. However, this contrast in the flap context distinguishes very few within-syntactic category minimal pairs (Wedel et al $2013 \mathrm{a}, \mathrm{b}$ ). As a consequence, the $/ \mathrm{t} \sim \mathrm{d} /$ contrast in this context contributes little to reducing uncertainty given idiosyncratic properties of the English lexicon, making maintenance of the cue for voicing in this context relatively less cost-effective for English speakers. Further in American English, high word predictability is correlated with reduction to the point of deletion of underlying $/ \mathrm{t}, \mathrm{d} /$ in that same intervocalic onset position, i.e., the flap context: apparent deletion is more probable in tokens of words that are more predictable given the following word (Raymond et al. 2006).

\subsubsection{Strong patterns in typologically weak positions}

We also observe strong patterns in typologically weak positions, such as at the end of words, when those positions play a particularly large role in message transmission due to languagespecific contextual, lexical, or morphological factors (as was the case in the toy example shown in Figure 7 above for the enhancement of word-final [t] in cat). Maltese, for example, displays robust patterns of contrast word-finally, such as the distinction between singleton and geminate consonants. While typologically rare, word-final geminate consonants in Maltese occur in the phonologically expected form of many words, as shown by the counts in (8) based on consonant type. 
(9) Type frequency counts for words with word-final geminate consonants (Hume, Rose \& Spagnol 2014)

\begin{tabular}{|l|l|l|l|}
\hline bb & 48 & $\iint$ & 99 \\
\hline $\mathrm{tt}$ & 713 & $\mathrm{~d} 3 \mathrm{~d} 3$ & 233 \\
\hline $\mathrm{dd}$ & 116 & $\mathrm{~mm}$ & 213 \\
\hline $\mathrm{kk}$ & 216 & $\mathrm{nn}$ & 243 \\
\hline ?? & 55 & $\mathrm{rr}$ & 105 \\
\hline ss & 982 & 11 & 704 \\
\hline
\end{tabular}

As discussed in Section 4.3.1, investing resources to maintain distinctions in word-final position is not generally beneficial given their weak contribution to uncertainty reduction. This is not the case in Maltese, however, due to specific properties of the language system. This includes the observation that the contrast between singleton-geminates for coronal stops in word-final position serves a morphological function with a high functional load, e.g. ghajjat ['e:j:et] 'he shouted' vs. ghajjatt [e'j:et:] 'I shouted' (see Blevins \& Wedel 2009 for a review of morphologically conditioned maintenance of contrast). We note that in Maltese, word-final syllables with consonant clusters and geminates are stressed, which independently provides these consonants with a platform that supports the perceptibility of their acoustic cues and thus the distinction between words (see §4.3.2). We also note that Maltese word-final geminates illustrate the conflict among the structural domains relevant to the distribution of weak/strong patterns; in this case, word-final position is strong prosodically yet weak in terms of lexical position. Together, these language-specific properties contribute to supporting the large inventory of contrasts in an otherwise lexically weak position. We leave the in-depth study of the interaction among different domains and its implications for the distribution of strong/weak patterns to future research.

\subsection{Summary}

In this section we have synthesized ideas from several fields of study to propose a unified account of both typologically common and typologically uncommon strong and weak phonological patterns. Our approach draws on well-established properties of spoken languages: (a) PUCs make different contributions to reducing uncertainty in message transmission, and (ii) common patterns in cue-types and their organization occur across the speech signal. The 
approach predicts that languages should evolve, on the one hand, to invest resources in contexts where phonological units will on average make a stronger contribution to uncertainty reduction and thus accurate message transmission, and on the other, to reduce resource cost in contexts where the contribution of PUCs to reducing uncertainty is on average weaker. These trends are predicted to hold cross-linguistically, and some of the factors that affect the contribution of particular PUCs to uncertainty are universally applicable, giving rise to a variety of typologically common phonological patterns. At the same time, other factors vary across languages, and while the trade-off principle between accurate message transmission and resource cost remains the same, the specific patterns that arise may be language-specific.

\section{Looking forward}

\subsection{Contributions to a general model of phonology}

In the previous sections, we have presented evidence that phonologies evolve in response to the interaction of biases toward accurate message transmission and low resource cost. For the purposes of our argument, we focused on patterns that have been traditionally framed in terms of a strong-weak continuum. However, the MOP framework predicts that all phonological patterns should evolve under the influence of these biases, and that any phonological pattern can be a consequence of these influences, because all patterns form part of messages. In the following, we briefly outline four examples that illustrate how the MOP framework applies to additional domains of phonology. A more in-depth discussion of these examples for specialist audiences is left to future work.

\subsubsection{Markedness}

Many recurrent patterns are typically referred to with respect to the cover term markedness, where typologically common patterns are assumed to be unmarked and less common patterns marked. Framed within the current approach, unmarked patterns are positioned within the diagonal area of relative stability in the figure; they effectively trade off accurate message transmission and resource cost. An interesting consequence of this approach is that both "strong" and "weak" patterns fall within this stable area and would thus be labeled unmarked, a noted paradox shown to be problematic for traditional approaches to markedness (Hume 2005). We 
illustrate these types of unmarked patterns and suggest how each would follow from the current approach (see also Hume 2016; Hume, Hall and Wedel 2016).

Consider first an unmarked "strong" pattern. Typologically, it is widely assumed that the unmarked position for consonants is in prevocalic, onset position (e.g. [ta]), as opposed to in postvocalic, coda position (e.g. [at]) (e.g. Clements \& Keyser 1985), consistent with the observation that languages generally have a broader range of consonant types in onset than coda position. As reviewed above in Section 4.3.2, MOP predicts this asymmetry is based in part on the fact that onset positions are on average closer to the beginning of the word than coda positions, and therefore contribute more on average to uncertainty reduction in lexical access. Further, prevocalic consonants typically have better phonetic cues to their identification than postvocalic consonants (§4.3.2); vowel formant transitions provide information about their place and manner of articulation, and for prevocalic stop consonants, a release burst provides cues to the segment's identity. Strong cues of this type support the accurate transmission of less predictable parts of a message. Conversely, producing bursts is not a physiological requirement of postvocalic stops. Consequently, under the assumption that production of a burst in postvocalic stops puts more demands on resource costs (e.g., because they take more time and/or effort to produce), coda release bursts (and/or other potentially redundant cues) are predicted to become phonologized in a language only when they are particularly beneficial to conveying messages. Both the assumption underlying this prediction and the prediction itself lend themselves to quantitative evaluation in future work.

Sound patterns in weak contexts have also been claimed to be unmarked. For example, Korean plain, aspirated, and tense consonants (e.g., [ $\left.\mathrm{t}^{\mathrm{h}} \mathrm{t}^{\prime}\right]$ ) contrast in onset position but are neutralized in coda position, where only the plain consonant occurs (e.g., [t]). Building on Trubetzkoy's (1969 [1939]) seminal work on phonological contrast, it is widely assumed in the phonological literature that the sound which occurs when a contrast is neutralized is the unmarked member of the opposition (Rice 1999). In MOP, common outputs of neutralization are hypothesized to effectively balance demands on resource cost with the segment's potential contribution to successfully communicating the message. That neutralization processes typically occur in typologically weak contexts is consistent with this prediction. Also consistent is the observation that PUCs that typically occur in neutralization contexts, such as the Korean plain consonant, are 
arguably articulatorily simple relative to the other PUCs that form part of the contrast set, suggesting that the former are lower in resource cost.

Note that, consistent with our discussion in Section 4.5, language-specific exceptions to these typologically common patterns are predicted to derive from properties of the language system itself, and are thus not exceptional from the perspective of their message-driven origins (see, e.g. Hume \& Tserdanelis 2003; Hume et al. 2013 regarding typologically uncommon unmarked patterns). The MOP approach to markedness can be tested cross-linguistically given the large existing body of literature on markedness patterns and diagnostics (see e.g., Rice 1999; Haspelmath 2006; Hume 2011).

\subsubsection{Epenthesis}

Epenthesis is commonly analyzed as the insertion of one or more sounds. For example, in some accounts of the English plural alternation $[-\mathrm{s}] \sim[-\mathrm{z}] \sim[-\mathrm{Iz}]$, the vowel in $[-\mathrm{Iz}]$ is treated as epenthetic occurring between a sequence of two strident consonants (Keyser \& O'Neil 1985). There is a large body of literature on the phonetics and phonology of epenthesis in native and non-native speech (e.g., Itô 1989; Paradis \& LaCharité 1997; Dupoux et al. 1999; Fleischhacker 2001; Uffmann 2007; Davidson 2006; Kim \& Kochetov 2011; N. Hall 2013; see N. Hall 2011 for a general overview) and based on this research it is generally assumed that the goal of epenthesis is to repair illicit structures, e.g. strings of consonants which do not occur in the particular language (N. Hall 2011). Re-conceptualized within MOP, epenthesis should serve in part to support accurate message transmission. Specifically, adding epenthetic structure to illicit sequences should reduce uncertainty about the message more than other alternatives, given the resource cost expended. Experimental evidence is consistent with this: epenthesis often results in a sequence being produced and processed more quickly and accurately (e.g., Donselaar et al. 1999; Kuipers et al. 1996; Davidson 2006) suggesting that epenthesis simultaneously reduces resource cost and improves message transmission accuracy.

Many languages exhibit optional or gradient epenthesis in particular contexts. If epenthesis serves to increase the likelihood of accurate message transmission, we expect optional epenthesis to occur preferentially in messages with greater uncertainty. This is observed in Dutch where variable vowel epenthesis is conditioned by word predictability, where less predictable words are more likely to include an epenthetic schwa, e.g. melk [mзlk] [m3lək] 'milk' (Tily \& Kuperman 
2011). Note that the Dutch consonant sequences affected by epenthesis are not in fact phonotactically ill-formed in the language, in line with the proposal that the function of epenthesis goes beyond "repairing" illicit structure, contrary to traditional assumptions regarding epenthesis (see, e.g. N. Hall 2011); rather epenthesis may facilitate the transmission of the message in which the structure occurs.

The proposal that modifications to the speech signal are also biased toward low resource cost leads to a further prediction: when multiple options for enhancing a message through epenthesis are available, the current perspective predicts that the preferred option will require the least investment in additional resources, all else being equal. This is consistent with evidence indicating that epenthetic segments are generally articulatorily simple (N. Hall 2011), and highly predictable in the epenthetic context or language more generally (Bromberg \& Hume 2005; Cristófaro-Silva \& Almeida 2006; Hong 2011; Hall \& Hume 2015; Hume et al. 2013). We would also expect the structure resulting from epenthesis to be similar to the non-epenthetic form since it will then be close to the expected form and therefore more easily recoverable by the listener. This prediction receives initial validation from Fleischhaker's (2001) findings that when listeners are given the choice of more than one site for epenthesis, e.g. /CCV/ > [_CCV] vs. [C_CV], they show a preference for the form that is closest auditorily to the original (non-epenthetic) form (see also Steriade 2008; Parlato-Oliveira et al. 2010; and $\$ 6.3 .1$ for related discussion). Note that while cross-linguistically, vowels that most commonly fit these criteria include mid central vowels such as schwa [ə] or high unrounded vowels such as [i i] (N. Hall 2011), typologically marked vowels can also satisfy these conditions, as is the case of French where the epenthetic vowel is the typologically uncommon mid front rounded vowel (Hume et al. 2013). We predict similar findings regarding epenthetic consonants.

\subsubsection{Assimilation}

Another common phonological process, assimilation, is characterized by one sound (the trigger of assimilation) extending a phonetic cue or phonological feature to another sound (the target of assimilation) within some domain. In English this can be observed as a variable process across morpheme and word boundaries where coronal nasals assimilate to the place of articulation of a following consonant, e.g. brow [n] bag brow [m] bag (e.g. Lahiri \& Reetz 2002; Dilley \& Pitt 2007), or as a lexicalized pattern within words involving labial, coronal and velar nasals, e.g. 
$u[m b] r e l l a, u[n d] e r$, si[yg]le. From the current perspective, we can think of assimilation as decreasing redundancy of the target while increasing redundancy of the trigger. Continuing our example, the nasal target of assimilation loses one or more cues to its original place of articulation, while the trigger gains redundancy given that its phonetic properties are extended to the preceding nasal, thus providing cues earlier in the signal to the trigger and its following material (see Otake et al. 1996 for evidence from Japanese).

The MOP framework predicts that assimilation arises in part because its occurrence supports effective information transfer given the trade-off between accurate message transmission and resource cost. Since the cues to the assimilation trigger are increased, while those to the target are reduced, we expect the trigger to occur in a position that provides a greater average contribution to accurately conveying messages, and the target to be in a context where the average contribution is smaller. Typological patterns are consistent with this prediction: targets of assimilation commonly occur in typologically weak positions (word-final/coda/preconsonantal) while triggers tend to occur in strong positions (word-initial/onset/prevocalic) (see e.g. Ohala \& Ohala 1993; Beddor \& Evans-Romaine 1995; Jun 1995, 2011; Boersma 1998; Kawahara \& Garvey 2014).

Additional support for MOP comes from cases in which only a subset of potential nasal targets undergo assimilation. We saw this above for English where coronal nasals tend to undergo place assimilation more so than velar and labial nasals. Viewed from a MOP perspective, we would expect the average contribution of the coronal nasal to message transmission to be lower than that of the other two. We find some support for this prediction from usage patterns in conversational English speech (Pitt et al. 2007) which indicate that the word-final coronal nasal occurs at a significantly higher rate than the labial or velar: (token frequency per million) /n/ 104,744.46;/m/ 46,723.5;/y/ 31,513.41. This suggests that coronal nasals are more predictable than labial and velar nasals (though this should be tested more directly) and thus more recoverable in contexts of assimilation, consistent with the findings in Lahiri \& Reetz (2002) for Dutch. As a result, we predict avoidance of nasal place assimilation only when nasal place cues themselves contribute strongly to accurate message transmission, such as when the place of a word-final nasal in the phonologically expected form is less predictable from context (see Turnbull et al., in prep). Indeed, to the extent that crosslinguistically the coronal contributes on average less to message transmission accuracy than other 
consonant place types, our approach correctly predicts that more languages would be expected to show neutralization patterns involving coronals than other places. ${ }^{23}$

While reducing coronal consonants is typologically more common than reducing other consonant types, the MOP framework predicts that coronal place may be preferentially preserved if the average contribution of coronal consonants to message transmission accuracy happens to be large. In Sri Lankan Portuguese Creole, for example, the labial nasal consonant undergoes place assimilation to a following consonant while the coronal nasal does not (Hume \& Tserdanelis 2003; see also Hume 2003). As expected, the coronal consonant is less predictable in the language than the labial.

\subsubsection{Boundaries}

As observed in the case of assimilation above, phonological patterns often occur across boundaries that separate words and other units of meaning, and can render boundaries more, or less identifiable. Our approach predicts that phonological patterns will be biased toward making boundaries between messages (e.g., words or morphemes) clearer if the degree of uncertainty associated with the message is high, to the extent that accurate boundary identification contributes to processing. On the other hand, when a message is predictable from context, having a clear onset and/or offset is less crucial. Liaison in Laurentian (Canadian) French (LF) illustrates. French liaison is a well-known sound pattern in which a consonant, typically the otherwise silent final consonant of a word $\left(\mathrm{W}_{1}\right)$ can surface as the onset of the vowel-initial syllable of the following word $\left(\mathrm{W}_{2}\right)$, e.g. le gros $[\mathrm{z}]$ enjeu, 'the big stake'. The liaison consonant straddles the two words, modifying the otherwise vowel-initial onset of W2. Côté (2013)'s study of conversational speech shows that liaison is more likely in cases where the syntactic category of $\mathrm{W}_{2}$ is predictable given $\mathrm{W}_{1}$; that is, where a given word occurs almost invariably before the same type of word. If liaison masks the boundary at the beginning of W2, which is typically a

\footnotetext{
${ }^{23}$ A link can be made between the current approach and formal phonological theories that represent the special character of coronals through feature underspecification (e.g. Kiparsky 1984; Archangeli 1984; Paradis \& Prunet 1992; Avery \& Rice 1992; Lahiri \& Reetz 2002). Lahiri \& Reetz (2002), for example, propose that coronals, which undergo place assimilation, are lexically underspecified for the feature [coronal] while labials and velars, which do not undergo assimilation, are specified for place features. Underspecification can be viewed as a formal device intended to express differences in the contribution of a given feature/segment to distinguishing messages in the language. For example, non-contrastive features - that is, those that do not distinguish words - are typically underspecified since they are predictable (Archangeli 1984; Clements 1988; Steriade 1987).
} 
strong position lexically, the MOP framework correctly predicts the avoidance of liaison in contexts in which W2 is less predictable. More generally, given the rich literature on sound patterns involving multiple units of meaning from morpheme to utterance, boundary phenomena provide a rich testing ground for the predictions of a message-based approach.

\subsection{Tests of the MOP framework}

Testing and extending the MOP framework requires in-depth analyses of individual languages as well as broad cross-linguistic studies. Because any grammatical system contains traces of its idiosyncratic history, single examples cannot provide convincing evidence either for or against a broader framework such as this. Instead, evaluations of the MOP framework require quantitative studies (ideally over larger cross-linguistic databases) that assess whether the statistical tendencies predicted by it are borne out (see, for example, the cross-linguistic strategy in Wedel et. al (2013) for studying the effect of functional load on predicting phoneme mergers; see also Graff 2012; Graff \& Jaeger 2009; Piantadosi et al. 2011, 2012). Further, the abstract framework we have outlined here provides us with hypotheses about the range of properties that language should exhibit, but does not a priori tell us what mechanisms will realize these properties, nor at what levels of organization. As a consequence, developing the MOP framework will require a broad empirical approach to both refine and test these hypotheses. In the remainder of this section, we note some of the outstanding questions and predictions generated by this approach, many of which are reflected in current research programs across the fields of linguistics and psychology, and suggest that the reasoning underlying MOP may provide insight into a broad range of issues in phonology and other domains.

One such area concerns classical phonological concepts and phenomena. Examples from the previous section show that reconceptualizing traditional phonological notions within the MOP framework can shed light on long-standing issues. What about other phonological concepts? The notion of minimal pair, for example, has been shown to be a predictive measure of phonetic enhancement/reduction in a number of the studies cited above. Does this suggest that the minimal pair is a privileged cognitive category in phonology? Here, we have argued that signal-specificity relationships influence phonetic enhancement/reduction processes, which in turn suggests that the minimal pair as traditionally defined may simply be a convenient measure which approximates these relationships well in many languages (see, for example, Burchill \& Jaeger 
2016, Martin \& Peperkamp 2016, and Wedel et al 2013a,b for approaches to functional load that go beyond minimal pairs as traditionally defined). In particular, less morphologically analytic languages (such as polysynthetic languages) often have relatively few minimal morpheme pairs as traditionally defined (though see cf. Bennett \& Tang 2016). Enhancement/reduction processes in these languages may provide test cases to refine our understanding of how competition between messages with similar signals can shape phonological patterns. Relatedly, the MOP framework predicts that all else being equal, a phoneme contrast that more often appears near the beginnings of minimal pairs should contribute to robustness more than one appearing more often near the ends of minimal pairs (for evidence from comprehension, supporting this view, see Magnuson et al. 2007). Can we develop and test a modified definition of minimal pair that takes this into account? (For steps in this direction, see also Cohen Priva 2010 and van Son and Pols 2003.)

While our focus in this paper has been on phonology, we expect MOP to also provide insight into other domains of linguistics. One extension of the ideas presented here would investigate the effective transmission of messages other than words and morphemes (see e.g., work in this spirit on word order, Gildea \& Jaeger, submitted; Maurits et al. 2010), including those that relate to higher-level units or the transfer of social or pragmatic meaning. Another would look beyond changes to phonology and consider the predictions of the approach to phenomena involving a change in a language's lexicon or the context in which a message occurs; that is, changes represented along the horizontal axis in Figure 5. To what extent can we predict which part of the linguistic system is more likely to change?

Throughout this paper, we have remained agnostic about the specific nature of the mechanism(s) that underlie the trade-off between accurate message transmission and resource cost. As noted in footnote 10, there are on-going debates in phonetic and psycholinguistic research as to what how communicative pressures come to shape production preferences. According to one view, comprehension plays the role of a filter, preventing phonetic realizations that are not accurately understood from being replicated (e.g., Guy 1996; Lindblom 1990; Ohala 1988). This has been particularly clearly articulated in exemplar-based accounts: upon having been produced, an exemplar-i.e., a specific pronunciation-will only be stored by the interlocutor, if it is recognized with sufficient confidence (Blevins \& Wedel 2009; Pierrehumbert 2001, 2002; Wedel 2006, 2012). A competing, though not incompatible, view holds that speakers 
implicitly predict the communicative consequences of their (intended) productions (Buz \& Jaeger in prep; Lindblom 1990; Jaeger 2013; e.g., by means of forward models, Jaeger \& Ferreira 2013) and/or that speakers learn from pervious miscommunications, adapting their subsequent production accordingly (e.g., Buz et al. 2016, in prep; Schertz 2013; Stent et al. 2008). This line of work has found, for example, that speakers seem to be able to hyper-articulate specific phonological contrasts in contexts where this very contrast was previously misunderstood (Buz et al. 2016, in prep). How these mechanisms interact and to what extent each of them contributes to the bias toward cost-effective, but accurate, message transmission that - according to MOP-is reflected in phonological systems remains an important area for future research.

Relatedly, other lines of research in psycholinguistics have pointed out that not everything that looks like a communicative effect necessarily is one (e.g., Arnold 2012; Baese-Berk \& Goldrick 2009; Bard et al 2000). For example, some have attributed the hyper-articulation of words with minimal pair neighbors to competition during production planning, rather than to an attempt to make a priori more confusable words easier to understand (e.g., Baese-Berk \& Goldrick 2009; Fox et al. 2015; Goldrick et al. 2013; Kirov \& Wilson 2013; for problems with this specific proposal, see Buz \& Jaeger 2016; Buz et al. 2016, in prep). A recent review of this and related debates - and why these alternative views are insufficient to explain existing evidence - is provided in Jaeger \& Buz (in press; for related discussion, see also Ernestus 2014). The validity of the MOP approach, of course, depends on the outcome of these debates, highlighting their relevance to phonological research.

\section{Relation to other theories and frameworks}

As noted at the outset, the proposed message-oriented approach to phonology (MOP) draws on evidence from a range of fields and benefits from a long lineage of observations, hypotheses, and theories. In this section we consider previous work in phonology situated in the same major bodies of work that our proposal springs from: (i) communication-based accounts, and (ii) variationist/usage-based/evolutionary models of language change. Following that, we discuss

related strands of work in other phonological traditions including positional faithfulness and phonetically-grounded approaches to phonology, including dispersion. 


\subsection{Communication-based Approaches}

Some of the most closely related antecedents to the current work are approaches that (1) directly incorporate communicative biases and (2) have an explicit role for diachronic pathways of change leading to synchronic variability. In particular, the current proposal can be understood as a claim that a bias toward accurate message transmission through the reduction of uncertainty is among the set of biases that shape diachronic changes that lead to synchronic patterns.

As reviewed in Section 3 above, effective communication systems trade-off the probability of accurate message transmission and resource cost, adding redundancy when message uncertainty is higher, and reducing it when uncertainty is lower (e.g., Zipf 1932; Jakobson, Cherry \& Halle 1952; Martinet 1972; Lindblom 1990; Silverman 1997, 2012; Boersma 1998; Bybee 2001; Kingston 2007; Kirby 2010; Harris 2011; Aylett \& Turk 2004; Levy \& Jaeger 2007). One of the key threads we have built upon in this work is Lindblom's seminal 1990 paper on hyper- and hypo-articulation or "H\&H" theory, which Lindblom based on a similarly motivated set of hypotheses about language production and processing. These hypotheses may be paraphrased as: (i) lexical access is grounded in discrimination among acoustic signals for lexical items, not signal invariance; (ii) discrimination among signals for lexical items is influenced by context; (iii) speaker output is listener-oriented in some fashion, favoring discriminability; (iv) speaker output is also speaker-oriented, favoring low speaker resource cost. On the basis of these hypotheses, Lindblom noted that the optimal degree of effort invested in the production of phonetic cues should vary by degree of predictability from context, and that if speakers are sensitive to this predictability, they should deviate accordingly in their productions. In H\&H theory, Lindblom proposed that these deviations should influence the evolution of phonological patterns. At the time, however, limitations in data and analytical tools made broader development and testing of this idea difficult. Over the last quarter century, however, advances in theory, experimental techniques, statistical methods, and corpus approaches are allowing us to develop and test more articulated hypotheses springing from this general set of insights. Our goal in this paper has been to show how findings arising through these advances have allowed us to build a

more specific formal framework couched in Bayesian inference for how a bias toward effective communication of meaning-based units influences the formation of phonological patterns, which simultaneously makes clear and testable quantitative predictions. 
Another key aspect of our proposal is that language users have access to statistical information about their language, and that predictability and expectation influence the shape of linguistic sound systems (cf. Zipf 1932; Jurafsky et al. 2001; Hume 2005; Kirby 2010; Cohen Priva 2012; Hume \& Mailhot 2013; Seyfarth 2014; Piantadosi et al. 2011). For example, the current work has strong ties to Zipf's (1932) seminal paper on the role of frequency in language. In that work he proposed the Principle of Relative Frequency which states that "the accent or degree of conspicuousness of any word, syllable, or sound is inversely proportionate to the relative frequency of that word, syllable, or sound, among its fellow words, syllables, or sounds in the stream of spoken language." Conspicuousness, for Zipf, is inversely correlated with the articulatory difficulty and acoustic salience of a sound, and is thus related to our use of the term resource cost. Zipf also recognized the interaction between predictability and resource cost: "any element of speech which occurs in spoken language more frequently than some other similar element demands less emphasis or conspicuousness than that other element which occurs more rarely. In other words, what is expected does not need or receive the emphasis or conspicuousness of the relatively less expected." The current approach builds on these foundational insights to develop an explicit model of the interaction between message predictability and resource cost. Similar to Zipf, we assume that lexical frequency plays a role in contributing to predictability, however, frequency alone is not sufficient to account for the full range of patterns; a word's predictability in context is crucial (see also Jaeger \& Buz in press; Piantadosi et al. 2011).

\subsection{Variationist/Usage-based/Evolutionary models of sound change}

As discussed in Section 3, speakers are sensitive to predictability in context, and adjust signals accordingly to balance the probability of accurate information transmission and resource cost. In Section 4, we argued that consistent, utterance-level adjustments to signals shape change in the overall phonological system. However, in order for utterance-level behavior in individuals to influence the long-term evolution of lexical and phonological patterns in speech communities, there must be mechanistic pathways that causally link these two vastly different time-scales. A large interconnected body of work has theorized and provided evidence for just such a general set of mechanisms. A diverse family of approaches, which we will collect under the term, 'variationist / usage-based / evolutionary' (VUE), is grounded in evidence that inherent biases in 
production, perception, and learning operate continually to influence the range of variants that arise and propagate through a speech community, and that the synchronic properties of a given phonological system arise in part through these processes (e.g., Baudouin de Courtenay 1895; Zipf 1932; Martinet 1955; Ohala 1989; Phillips 1984; Lindblom 1990; Labov 1994; Nettle 1999; Bybee 2001, 2002; Pierrehumbert 2003; Blevins 2004; Wedel 2007, 2012, Mielke 2008; Beddor 2009; Hay \& Maclagan 2012, Kirby et al. 2015). A prediction of many VUE approaches that is relevant to the current proposal is that a bias towards a given phonological pattern in some words, some of the time can be sufficient to drive the generalization of that pattern in the phonology of a speech community.

To arrive at this prediction, modern VUE approaches draw on two related findings: (i) linguistic categories maintain some record of experienced variation rather than being fully abstract (reviewed in the context of language in, among many others, Baayen 2007; Bybee 2002; Ernestus 2011; Goldinger 1996, 1998; Johnson 1997, 2006; Pierrehumbert 2001, 2002; Pisoni \& Levi 2007; Strand \& Johnson 1999; Warker \& Hay 2011; for recent reviews specifically in the context of exemplar models, see Foulkes \& Hay 2015; Weatherholtz \& Jaeger in press; Wedel 2007), and (ii) experiencing a particular category variant influences future production and perception behavior not only for that category, but also for similar categories (reviewed in Goldinger 2000; Pierrehumbert 2002, 2003; Kleinschmidt \& Jaeger 2015; Wedel 2012; see also Nielsen 2007; Pardo 2006; Eisner \& McQueen 2005; Kraljic \& Samuel 2005a, 2005b). This production-perception loop (Pierrehumbert 2001; Oudeyer 2002) provides a pathway by which variation in cues to linguistic category membership can spread across categories, from word to word (e.g., Bybee 2002b; Phillips 2006; Wang 1969) and from sound to sound (e.g., Kraljic \& Samuel 2005a; Mielke 2008; Wedel 2006) over lifetimes and generations within a speech community. As children abstract phonological grammars from the input they hear, what started as gradient, token-level shifts in production behavior can become reinterpreted as categorical patterns, completing the link from usage at the level of the individual, to phonology at the level of the language (e.g., Beddor 2009). The proposal we have made here is grounded in the general VUE prediction that if speakers pronounce phonetic cues to words more clearly when the word is less likely to be correctly recognized by a listener, lexical (Manin 2006; Piantadosi et al. 2011; Seyfarth 2014) and phonological patterns (Cohen Priva 2012; Hay et al. 2015; Wedel, 2006, 2012) should evolve that reflect this bias. 
The MOP framework is situated within this previous body of work, but moves us toward a more explicit model of language variation and change by introducing strongly constraining - and testable - predictions. In much previous work, probabilistic quantities assumed to predict usage effects are often left unspecified or chosen based on convenience (e.g., frequency, conditional probability). Instead, because MOP is grounded in established theoretical frameworks for quantifying uncertainty and information in terms of contextual predictability (see footnote 14 for related comments), it allows us to make more specific and constrained predictions about the role of probabilistic inference in accurate message transmission in usage. MOP is also distinctive in that variability is driven by the cost-effective informativity of meaning-bearing units. As we have argued here, this approach opens a new field of nuanced, testable hypotheses about languagespecific and language-general phonological patterns.

\subsection{Other relevant approaches}

While the above section has outlined the closest antecedents of the current approach, there are many other theories that have directly influenced the ideas presented here. While it is impossible to engage in a full discussion of all of them, we present a brief discussion of the relation of the current approach to several other precursors: phonetically-grounded phonology, various versions of dispersion theory, and insights from the OT-based notion of positional faithfulness.

\subsubsection{Phonetically grounded phonology}

Within phonological theory, there is a long tradition of accounting for sound patterns through reference to production and perception factors (see e.g., Stampe 1979; Archangeli \& Pulleyblank 1994; Flemming 1995; Jun 1995; Boersma 1998, 2011; Hume 1998; Hume \& Johnson 2001; Kirchner 2001; Kirchner, Hayes \& Steriade 2004). The current work obviously builds on this tradition. Similar to our predecessors, MOP assumes that a sound's physical quality, determined by its inherent nature and the context in which it occurs, contributes in important ways to shaping its behavior within a language system.

Yet our proposal also differs significantly from phonetically-based phonological accounts. To illustrate, consider the cross-linguistically common pattern of word-final obstruent devoicing, e.g. German [hundə] 'dog, pl.' [hunt] 'dog'. Working within an Optimality-theoretic 
framework, Steriade (2001) proposes a perceptually grounded constraint against the voicing of obstruents in word-final position (see (10)).

(10) Phonotactic constraint (Steriade 2001)

*[+VOICE]/_ $]_{\text {WORD }}$ : voiced obstruents do not occur at the end of the word.

In languages where this constraint is phonologically active, input forms that have final voiced obstruents, e.g. /taeb/, would need to be repaired in some way. Several potential grammatical responses (repairs) are postulated (op. cit.) to be available, including devoicing: /taeb/ $\rightarrow$ [taep], nasalization: /taeb/ $\rightarrow$ [taem], V-insertion: /taeb/ $\rightarrow$ [taebə], and segment reversal: $/ \mathrm{taeb} / \rightarrow$ [baet]. However, most of these patterns are absent cross-linguistically; instead, there is an optimal repair of devoicing which is distinguished from other grammatically plausible repairs because, Steriade argues, it is the one where the change would be the least noticeably different from the input form. It is for this reason then, that devoicing is argued to be optimal in the particular instance of word-final voiced obstruents.

Yet, why does being the least noticeably different matter? Why do languages tend to avoid structures like word-final voiced obstruents? And why is such a repair necessary in the first place? MOP attempts to provide a deeper understanding of such questions. With respect to the latter one, under the assumption that phonological changes emerge through the transmission of messages, the reason why languages tend to avoid a certain structure is that their potential to reduce message uncertainty does not justify the investment in resources. As an example, consider German as compared to English: the number of minimal pairs that would hypothetically hinge on a voicing contrast in word-final obstruents in German is much smaller than the number in English (Wedel et al., in prep). Even in dialects of English that undergo final devoicing under pressure from German, the lexical contrast is maintained (e.g., Purnell et al. 2005). Thus, it appears that the high importance of the contrast in English makes it worth investing in, whereas in German, the investment does not have a large enough lexical payoff to be worthwhile. The preference for outputs to be least noticeably different (or perceptually inconspicuous, Kohler 1990) also has direct links to the current proposal; it means being not noticeably different from the lexical entry, i.e., the phonologically expected message, which is relevant only because it is the communication of that message that is intended. 
Thus, in the current approach the common avoidance of final voiced obstruents can be seen as resulting from two interacting observations. First, word-final positions are, generally speaking, weak positions in terms of the amount of information they have the potential to contribute to accurately transmitting the message. Second, the cost of maintaining voicing in obstruents is physiologically demanding due to the difficulty in maintaining a pressure differential across the vocal folds while obstructing airflow in the oral cavity (Kewley-Port \& Preston 1974). Thus, a voiced obstruent in final position will, as a general proposition, be located toward the top lefthand corner of Figure 5: it is costly in terms of production, and, because it is in word-final context, it is likely to contribute relatively little to accurately transmitting the message. Consequently, unless the voicing distinction in a word-final obstruent contrast distinguishes many lexical competitors, as in English, it is predicted to be unstable and prone to reduction, as in German.

Other "repairs" proposed to satisfy the constraint in (10) such as nasalization, V-insertion, and segment reversal, tend to increase redundancy: cues are added in V-insertion and nasalization, or maintained in a different context (segment reversal). From a MOP perspective, it is not the category of voicing that is being avoided in a constraint like *[+VOICE $] /]_{\text {word}}$, but rather the cost of producing voiced obstruents in positions that contribute little to accurate message transfer; viable repairs will only be ones that reduce cost relative to communicative importance and thus there's no reason to consider repairs that increase redundancy (see also Boersma 1998, 2011, and papers cited therein, for an Optimality Theory-based, but emergent, approach to such phenomena).

In languages where final voiced obstruents are not reduced, as in English, or are even enhanced, MOP predicts that, all else being equal, they will on average be found to have a relatively higher functional load over the lexicon and word-finally, in particular; that is, they will tend to distinguish a larger number of lexical competitors. In this case, the word-final voicing contrast would be situated closer to the upper right corner of Figure 5, rather than toward the upper left. As always, it is important to remember that like all VUE-type approaches, the current approach is non-deterministic: there are multiple pathways to change, and single cases can neither prove nor disprove a theory. Instead, the strongest evidence comes from statistical trends across larger sets of languages. 


\subsubsection{Dispersion}

Another major precursor to the current approach is the notion of dispersion, discussed by a number of researchers working in somewhat different frameworks (e.g., Lindblom 1990; Flemming 2002; Boersma \& Hamann 2008; Padgett 2009; Ní Chiosáin \& Padgett 2010; D. Hall 2011). The central idea in what we will refer to as "classic" dispersion theories (e.g., Flemming 2002; Padgett 2009) is that elements within phoneme inventories have a tendency to be relatively far apart from each other, i.e., maximally distinct, though this pattern is constrained by other considerations such as minimizing articulatory effort and maximizing the total number of contrasts. There are obvious corollaries to the current approach, including the stated motivation for dispersion-for example, Flemming (2004: 232) says: "The need to avoid confusion is hypothesized to derive from the communicative function of language. Successful communication depends on listeners being able to recover what a speaker is saying. Therefore it is important to avoid perceptually confusable realizations of distinct categories; in particular, distinct words should not be perceptually confusable." Thus, dispersion theories are often rooted in a view of language as communication, and specifically recognize the trade-off between a need for robust message transmission (maximizing distinct contrasts) and a need for cost reduction (minimizing articulatory effort) similar to the approach here.

Note, however, that in the classic version of dispersion, the stated goal is in fact to maximize the distinctiveness of contrasts which, like the goal of avoiding final voiced obstruents expressed through the $*[+\mathrm{VOICE}] /]_{\text {WORD }}$ constraint $(\$ 6.3 .1)$, creates a somewhat artificial teleology that does not reflect the actual biases that are shaping the system, a weakness pointed out by both Boersma \& Hamann (2008) and D. Hall (2011). The former suggest that dispersion is instead emergent from the diachronic interaction of perceptual and articulatory considerations. Hall (2011) suggests that dispersion is emergent from the synchronic interaction of minimal phonological representations and phonetic enhancement, where enhancements "amplify the salience" of contrasts in the system. The current approach shares the functional insights of both classic dispersion and Boersma \& Hamann (2008), along with the implicit motivation for phonetic enhancement in Hall (2011), while pushing the level of explanation further back. That is, the current approach takes seriously the idea that speech is about accurate message transmission, which leads inevitably to the conclusion that the system will be modulated in ways that enhance robustness. 
Furthermore, D. Hall's (2011) view that the fundamental source of dispersion is the contrastive system of the language is consonant with the current point that phonological systems are about encoding lexical contrasts. Unlike all of these theories, however, the current proposal is not sound-centric. That is, previous theories focus on the inventories of individual sounds and the need for those sounds to be distinct from one another, while MOP focuses on the modification of sounds in response to biases on information transmission imposed upon the morpheme, word or higher units of meaning in which the sounds occur $(\S 4.4)$. Dispersion is thus not investigated in isolation. Rather, a language's phonological system is taken to exist within a larger system of communication which can strongly influence the development and shape of its sound patterns.

\subsubsection{Positional faithfulness}

In addition to the phonetic-based accounts discussed above, our approach also draws on insights from psychologically-grounded accounts. The latter line of research has focused on the use of psycholinguistic evidence to elucidate asymmetrical sound patterns such as those discussed in this paper (e.g., Beckman 1998; Hume 1998; Mielke \& Hume 2001; Smith 2004). Beckman (1997:8), for example, acknowledges that certain positions (such as onsets) share processing advantages independently of their phonetic characteristics: "Phonological contrasts are preferentially maintained in prominent positions because these positions are exactly those which take priority in perception and processing." To formalize such patterns within OT, positional faithfulness constraints are introduced, such as the constraint IDENT-ONSET(VOICE), which favors the maintenance of voicing in onset position. By crucially ranking the positional constraint above the markedness constraint, ${ }^{*}[\mathrm{VOICE}]$, which penalizes the presence of the feature [voice], and this markedness constraint above the non-positional faithfulness constraint IDENT(VOICE), a voicing alternation is generated in which voicing is maintained only in onset position (see Beckman 1998 for discussion).

(11)a. IdEnT-OnSET(VOICE): Onset segments and their input correspondents must agree in voicing.

b. *VDOBSTR: Voiced obstruents are prohibited.

c. IDENT(VOICE): Segments and their input correspondents must agree in voicing.

d. IDENT-OnSET(VOICE) $>>*$ [VOICE] $>>$ IDENT(VOICE) 
Differences between our proposal and positional faithfulness accounts are similar to those noted above for phonetically-grounded approaches. MOP aims to understand why certain positions are advantageous for processing and perception and why others are not. Specifically, positions that have a high potential aggregate contribution to accurate message transmission are those that will emerge as strong positions, and be associated with more redundancy. A further difference in approaches relates to the level of explanation. In positional faithfulness accounts, pressure exerted on sounds leads to phonological change and consequently, the modification of words and higher-level units. Conversely, we propose that the impetus for change comes from communicative constraints affecting the transmission of meaning-based units such as words which leads to modulation of redundancy in the signal.

Crucially, MOP also differs from the phonological approaches discussed above in providing the mathematical foundation to derive quantitative predictions about sound patterns thereby moving toward a more rigorous and parsimonious approach.

\section{Conclusion}

The arrow of science is propelled forward by the repeated interplay between theory, which generates hypotheses, and experiment, which tests them. Every theory is therefore a rich accretion of earlier proposals and experiments that collaborate together to give us a theory in its current form. Likewise, the synthesis that we have articulated here draws on a deep and diverse body of work, as discussed above. Weaving these threads together, we have suggested that by taking seriously the view of language as a system of message transmission, we are able to make strong and substantially supported predictions about the shape of language. In particular, we predict that the trade-off between biases toward accurate message transmission and lower resource cost should interact to promote the development of phonological systems that are both communicatively robust and efficient. Significantly, we show that within this message-oriented approach to phonology, established strong-weak patterns in phonology are only consistent with the conclusion that these biases are primarily focused at the level of meaning-bearing units such as the morpheme or word, rather than at the sub-lexical level. 


\section{References}

Ackerman, Farrell, James P. Blevins \& Robert Malouf. 2009. Parts and wholes: Implicative patterns in inflectional paradigms. Analogy in grammar: Form and acquisition. 54-82.

Allopenna, Paul D., James S. Magnuson \& Michael K. Tanenhaus. 1998. Tracking the time course of spoken word recognition using eye movements: Evidence for continuous mapping models. Journal of Memory and Language 38.419-39.

Archangeli, Diana. 1984. Underspecification in Yawelmani phonology and morphology: Massachusetts Institute of Technology Doctoral dissertation.

Archangeli, Diana \& Douglas Pulleyblank. 1994. Grounded phonology Cambridge, MA: MIT Press.

Arnold, Jennifer E., Jason M. Kahn \& Giulia C. Pancani. 2012. Audience design affects acoustic reduction via production facilitation. Psychonomic Bulletin and Review 19.505-12.

Arnon, Inbal \& Neal Snider. 2010. More than words: Frequency effects for multi-word phrases. Journal of Memory and Language 62.67-82.

Aylett, Matthew. 2000. Stochastic suprasegmentals: Relationships between redundancy, prosodic structure, and care of articulation in spontaneous speech. Interspeech.646-49.

Aylett, Matthew \& Alice Turk. 2004. The Smooth Signal Redundancy Hypothesis: A functional explanation for relationships between redundancy, prosodic prominence, and duration in spontaneous speech. Language and Speech 47.31-56.

—. 2006. Language redundancy predicts syllabic duration and the spectral characteristics of vocalic syllable nuclei. Journal of the Acoustical Society of America 119.3048-58.

Baayen, R. H. 2007. Storage and computation in the mental lexicon. The Mental Lexicon: Core Perspectives, ed. by G. Jarema \& G. Libben, 81-104. Amsterdam: Elsevier.

Baese-Berk, Melissa \& Matthew Goldrick. 2009. Mechanisms of interaction in speech production. Language and Cognitive Processes 24.527-54.

Baudouin de Courtenay, Jan. 1972 [1895]. An attempt at a theory of phonetic alternations. A Baudouin de Courtenay Anthology: The Beginnings of Structural Linguistics, ed. by E. Stankiewicz, 144-212. Bloomington: Indiana University Press.

Bauer, Robert S., Kwan Hin. Cheung \& Pak Man Cheung. 2003. Variation and merger of the rising tones in Hong Kong Cantonese. Language Variation and Change 15.211-25.

Bayes, Thomas. 1763. An essay toward solving a problem in the Doctrine of Chances. Philosophical Transactions 53.370-418.

Beckman, Jill. 1997. Positional faithfulness, positional neutralisation, and Shona vowel harmony. Phonology 14.1-46.

-. 1998. Positional faithfulness. Amherst, MA: University of Massachusetts Doctoral dissertation.

Beckner, Clay, Richard Blythe, Joan L. Bybee, Morten H. Christiansen, William Croft, Nick C. Ellis, John Holland, Jinyun Ke, Diane Larsen-Freeman \& Tom Schoenemann. 2009. Language is a complex adaptive system: Position paper. Language Learning 59.1-26.

Beddor, Patrice \& David Evans-Romaine. 1995. Acoustic and perceptual factors in nasal place assimilation. Rivista di Linguistica 7.145-74.

Beddor, Patrice Speeter. 2009. A coarticulatory path to sound change. Language 85.785-821.

Bejjanki, Vikranth R., Jeffrey M. Beck, Zhong-Lin Lu \& Alexandre Pouget. 2011. Perceptual learning as improved probabilistic inference in early sensory areas. Nature Neuroscience $14.642-48$. 
Bell, Alan, Jason M. Brenier, Michelle Gregory, Cynthia Girand \& Daniel Jurafsky. 2009. Predictability effects on durations of content and function words in conversational English. Journal of Memory and Language 60.92-111.

Bell, Alan, Daniel Jurafsky, Eric Fosler-Lussier, Cynthia Girand, Michelle Gregory \& Daniel Gildea. 2003. Effects of disfluencies, predictability, and utterance position on word form variation in English conversation. Journal of the Acoustical Society of America 113.1001-24.

Bennett, Ryan \& Kevin Tang. 2016. Modelling Phonetic and Phonological Variation with 'Small' Data: Evidence from Kaqchikel Mayan. Paper presented at the 15th Conference on Laboratory Phonology, Cornell University.

Bergen, Leon, Noah D. Goodman \& Roger Levy. 2012. That's what she (could have) said: How alternative utterances affect language use. Proceedings of the 34th Annual Conference of the Cognitive Science Society.

Bicknell, Klinton, T. Florian Jaeger \& Michael K. Tanenhaus. 2016. Now or ...later: Language processing does not immediately forget perceptual data. Brain and Behavioral Sciences 39.23-24.

Blevins, James P. 2013. The information-theoretic turn. Psihologija 46.355-75.

Blevins, Juliette. 2004. Evolutionary Phonology: The Emergence of Sound Patterns Cambridge: Cambridge University Press.

Blevins, Juliette. 2006. A theoretical synopsis of Evolutionary Phonology. Theoretical Linguistics 32. 117-166.

Blevins, Juliette \& Andrew Wedel. 2009. Inhibited sound change: An evolutionary approach to lexical competition. Diachronica 26.143-83.

Blumstein, Sheila E. \& Kenneth N. Stevens. 1979. Acoustic invariance in speech production: Evidence from measurements of the spectral characteristics of stop consonants. Journal of the Acoustical Society of America 66.1001-17.

Bock, Kathryn \& Willem Levelt. 1994. Language production: Grammatical encoding. Handbook of psycholinguistics, ed. by M.A. Gernsbacher, 945-84. London: Academic Press.

Boersma, Paul. 1998. Functional phonology: formalizing the interactions between articulatory and perceptual drives The Hague: Holland Academic Graphics.

Boersma, Paul. 2009. Cue constraints and their interactions in phonological perception and production. Phonology in perception, ed. by P. Boersma \& S. Hamann, 1-24. Berlin: Mouton de Gruyter.

Boersma, Paul. 2011. A programme for bidirectional phonology and phonetics and their acquisition and evolution. Bidirectional Optimality Theory, ed. by A. Benz \& J. Mattausch, 33-72. Amsterdam: John Benjamins.

Boersma, Paul \& Silke Hamann. 2008. The evolution of auditory dispersion in bidirectional constraint grammars. Phonology 25.217-70.

Bouchard, Kristofer E., Nima Mesgarani, Keith Johnson \& Edward F. Chang. 2013. Functional organization of human sensorimotor cortex for speech articulation. Nature 495.327-32.

Brandão de Carvalho, Joaquim, Tobias Sheer \& Philippe Ségéral. 2008. Lenition and fortition Berlin: Mouton de Gruyter.

Browman, Catherine \& Louis Goldstein. 1989. Articulatory gestures as phonological units. Phonology 6.201-51.

Browman, Catherine P. \& Louis M. Goldstein. 1986. Towards an articulatory phonology. Phonology Yearbook 3, 219-52. 
Burchill, Zachary \& T. Florian Jaeger. 2016. Modeling new conceptions of functional load with perceptual confusability. Paper presented at the 15th Conference on Laboratory Phonology, Cornell University.

Buz, Esteban \& T. Florian Jaeger. 2016. The (in)dependence of articulation and lexical planning during isolated word production. Language, Cognition, and Neuroscience 31.404-24.

- . in prep. Hyperarticulation effectively reduces perceptual uncertainty despite noisy motor system.

Buz, Esteban, Michael K. Tanenhaus \& T. Florian Jaeger. 2016. Dynamically adapted contextspecific hyper-articulation: Feedback from interlocutors affects speakers' subsequent pronunciations. Journal of Memory and Language 89. 68-86.

-. In prep. Implicit causal inference during adaptive speech production.

Bybee, Joan L. 2001. Phonology and language use Cambridge: Cambridge UP.

- . 2002a. Phonological evidence for exemplar storage of multiword sequences. Studies in Second Language Acquisition 24. 215-21.

- 2002b. Word frequency and context of use in the lexical diffusion of phonetically conditioned sound change. Language Variation and Change 14.261-90.

Bybee, Joan L. \& Joanne Scheibman. 1999. The effect of usage on degrees of constituency: The reduction of don't in English. Linguistics 37.575-96.

Canfield, D. Lincoln. 1981. Spanish pronunciation in the Americas Chicago: University of Chicago Press.

Casali, Roderic F. 1996. Resolving hiatus. Los Angeles: UCLA Doctoral dissertation.

-. 1997. Vowel elision in hiatus contexts: Which vowel goes? Language 73.493-533.

-. 2011. Hiatus resolution. Blackwell Companion to Phonology, ed. by M. van Oostendorp, C.J. Ewen, E. Hume \& K. Rice, 1434-60. Oxford: Wiley-Blackwell.

Chang, Edward F., Jochem W. Rieger, Keith Johnson, Mitchel S. Berger, Nicholas M. Barbaro \& Robert T. Knight. 2010. Categorical speech representation in human superior temporal gyrus. Nature Neuroscience 13.1428-32.

Chen, Qi \& Daniel Mirman. 2012. Competition and cooperation among similar representations: Toward a unified account of facilitative and inhibitory effects of lexical neighbors. Psychological Review 119. 417-30.

Cherry, Colin, Morris Halle \& Roman Jakobson. 1953. Toward the logical description of languages in their phonemic aspect. Language 29. 34-46.

Cho, Young-mee Yu. 1990. Parameters of consonantal assimilation: Stanford University Doctoral dissertation.

Chodroff, Eleanor, Alessandra Golden \& Colin Wilson. 2016. An empirical and computational study of generalized adaptation to natural talker-specific VOT. Paper presented at the 15th Conference on Laboratory Phonology, Cornell University.

Chomsky, Noam. 1965. Aspects of the theory of syntax Cambridge, MA: MIT Press.

Christiansen, Morten H. \& Nick Chater. 2016. The now-or-never bottleneck: A fundamental constraint on language. Behavioral and brain sciences 39. 1-52.

Clark, Andy. 2013. Whatever next? Predictive brains, situated agents, and the future of cognitive science. Behavioral and brain sciences 36. 181-253.

Clayards, Meghan, Michael K. Tanenhaus, Richard N. Aslin \& Robert A. Jacobs. 2008. Perception of speech reflects optimal use of probabilistic speech cues. Cognition 108. 804-09.

Clements, George N. 1988. Toward a substantive theory of feature specification. Proceedings of NELS 18, ed. by J. Blevins \& J. Carter, 79-93. Amherst, MA: GLSA. 
Clements, George N. \& Samuel Jay Keyser. 1985. CV phonology: A generative theory of the syllable Cambridge, MA: MIT Press.

Coetzee, Andries W. 2002. Between-language frequency effects in phonological theory. University of Massachusetts Amherst.

Cohen Priva, Uriel. 2010. Constructing typing-time corpora: A new way to answer old questions. Proceedings of the 32nd Annual Conference of the Cognitive Science Society, ed. by S. Ohlsson \& R. Catrambone, 43-48. Austin, TX: Cognitive Science Society.

—. 2012. Sign and signal: Deriving linguistic generalizations from information utility: Stanford University Doctoral dissertation.

-. 2015. Informativity affects consonant duration and deletion rates. Laboratory Phonology 6. 243-78.

Cole, Desmond T. 1967. Some features of Ganda linguistic structure Johannesburg: Witwatersrand University Press.

Cole, Jennifer, Yoonsook Mo \& Mark Hasegawa-Johnson. 2010. Signal-based and expectationbased factors in the perception of prosodic prominence. Laboratory Phonology 1. 425-52.

Cole, Ronald A., Jola Jakimik \& William E. Cooper. 1978. Perceptibility of phonetic features in fluent speech. Journal of the Acoustical Society of America 64. 44-56.

Connine, Cynthia M., Dawn G. Blasko \& Michael Hall. 1991. Effects of subsequent context in auditory word recognition: Temporal and linguistic constraints. Journal of Memory and Language 30.234-50.

Cook, Susan Wagner, T. Florian Jaeger \& Michael K. Tanenhaus. 2009. Producing less preferred structures: More gestures, less fluency. The 31st Annual Meeting of the Cognitive Science Society, ed. by N.A. Taatgen \& H. van Rijn, 62-67. Austin, TX: Cognitive Science Society.

Côté, Marie-Hélène. 2000. Consonant cluster phonotactics: A perception-based approach: Massachusetts Institute of Technology Doctoral dissertation.

—. 2014. Liaison et assibilation en français laurentien. La liaison: Approches contemporaines, ed. by C. Soum-Favaro, A. Coquillion \& J.-P. Chevrot, 9-31. Berne: Peter Lang.

Cristia, Alejandrina \& Amanda Seidl. 2013. The hyperarticulation hypothesis of infant-directed speech. Journal of Child Language 41.1-22.

Cristófaro-Silva, Thaïs \& Leonardo S. Almeida. 2006. On the nature of epenthetic vowels. Paper presented to the Laboratory Phonology 10, Paris, 2006.

Cutler, Anne. 2010. Abstraction-based efficiency in the lexicon. Laboratory Phonology 1.301-18.

Cutler, Anne, John A. Hawkins \& Gary Gilligan. 1985. The suffixing preference: A processing explanation. Linguistics 23. 723-58.

Cutler, Anne \& Dennis Norris. 1988. The role of strong syllables in segmentation for lexical access. Journal of Experimental Psychology: Human Perception and Performance 14. 113-21.

Dahan, Delphine. 2010. The time course of interpretation in speech comprehension. Current Directions in Psychological Science 19.121-26.

Dahan, Delphine \& James S. Magnuson. 2006. Spoken word recognition. Handbook of psycholinguistics, ed. by M.J. Traxler \& M.A. Gernsbacher, 249-84. Amsterdam: Academic Press.

Davidson, Lisa. 2006. Phonology, phonetics, or frequency: Influences on the production of nonnative sequences. Journal of Phonetics 34. 104-37.

Degen, Judith, Michael Franke \& Gerhard Jäger. 2013. Cost-based pragmatic inference about referential expressions. Proceedings of the 35th Annual Meeting of the Cognitive Science Society. 
del Prado Martín, F. M., A. Kostić \& R. H. Baayen. 2004. Putting the bits together: An information theoretical perspective on morphological processing. Cognition 94. 1-18.

Dell, Gary S. \& Peter A. Reich. 1981. Stages in sentence production: An analysis of speech error data. Journal of Verbal Learning and Verbal Behavior 20. 611-29.

Dell, Gary S., Myrna F. Schwartz, Nadine Martin, Eleanor M. Saffran \& Deborah A. Gagnon. 1997. Lexical access in aphasic and nonaphasic speakers. Psychological Review 104. 801-38.

Dilley, Laura C. \& Mark Pitt. 2007. A study of regressive place assimilation in spontaneous speech and its implications for spoken word recognition. Journal of the Acoustical Society of America 122. 2340-53.

Dupoux, Emmanuel, Kazuhiko Kakehi, Yuki Hirose, Christophe Pallier \& Jacques Mehler. 1999. Epenthetic vowels in Japanese: A perceptual illusion? Journal of Experimental Psychology: Human Perception and Performance 25. 1568-78.

Eisner, Frank \& James M. McQueen. 2005. The specificity of perceptual learning in speech processing. Perception and Psychophysics 67. 224-38.

Ernestus, Mirjam. 2011. Gradience and categoricality in phonological theory. The Blackwell Companion to Phonology, ed. by M. van Oostendorp, C.J. Ewen, E. Hume \& K. Rice, 211536. Oxford: Wiley-Blackwell.

-. 2014. Acoustic reduction and the roles of abstractions and exemplars in speech processing. Lingua 142. 27-41.

Ernestus, Mirjam, R. H. Baayen \& Schreuder. 2002. The recognition of reduced word forms. Brain and Language 81. 162-73.

Farmer, Thomas A., Meredith Brown \& Michael K. Tanenhaus. 2013. Prediction, explanation, and the role of generative models in language processing. Behavioral and brain sciences 36 . 31-32.

Farmer, Thomas A., Alex B. Fine, Shaoron Yan, Spyridoula Cheimariou \& T. Florian Jaeger. 2014. Error-driven adaptation of higher-level expectations during reading. Proceedings of the 36th Annual Cognitive Science Society Conference. 2181-86.

Feldman, Naomi H., Thomas L. Griffiths \& James L. Morgan. 2009. The influence of categories on perception: Explaining the perceptual magnet effect as optimal statistical inference. Psychological Review 116. 752-82.

Ferreira, Victor S. \& L. Robert Slevc. 2007. Grammatical encoding. Oxford Handbook of Psycholinguistics, ed. by M.G. Gaskell, 453-70. Oxford: Oxford University Press.

Ferrer i Cancho, Ramon. 2005. Zipf's law from a communicative phase transition. The European Physical Journal B 47. 449-57.

Ferrer i Cancho, Ramon \& Albert Díaz-Guilera. 2007. The global minima of the communicative energy of natural communication systems. Journal of Statistical Mechanics 2007.P06009.

Fleischhacker, Heidi. 2001. Cluster-dependent epenthesis asymmetries. UCLA Working Papers in Linguistics 7. 71-116.

Flemming, Edward. 1995. Auditory representations in phonology: UCLA Doctoral dissertation.

-. 2002. Auditory representations in phonology London: Routledge.

-. 2004. Contrast and perceptual distinctiveness. Phonetically Based Phonology, ed. by B. Hayes, R. Kirchner \& D. Steriade, 232-76. Cambridge: Cambridge University Press.

—. 2010. Modeling listeners. Laboratory Phonology 10, ed. by C. Fougeron, B. Kühnert, M. D'Imperio \& N. Vallée, 587-606. Berlin: De Gruyter Mouton.

Foley, James. 1977. Foundations of theoretical phonology. Cambridge: Cambridge University Press. 
Foulkes, Paul \& Jennifer Hay. 2015. The emergence of sociophonetic structure. Handbook of Language Emergence, ed. by B. MacWhinney \& W. O'Grady, 292-313. Malden, MA: Wiley Blackwell.

Fox, Neal P., Megan Reilly \& Sheila E. Blumstein. 2015. Phonological neighborhood competition affects spoken word production irrespective of sentential context. Journal of Memory and Language 83. 97-117.

Frank, Michael C. \& Noah D. Goodman. 2012. Predicting pragmatic reasoning in language games. Science 336. 998-98.

Franke, Michael. 2009. Signal to act: Game theory in pragmatics: Institute for Logic, Language, and Computation Doctoral dissertation.

Franke, Michael \& Gerhard Jäger. 2015. Probabilistic pragmatics, or why Bayes' rule is probably important for pragmatics (ed.) U. Tübingen.

Frazier, Lynn. 1979. Sentence processing: A tutorial review. Attention and performance XII: The psychology of reading, ed. by M. Coltheart, 559-86. Hillsdale, NJ: Lawrence Erlbaum Associates.

Fricke, Melinda, Melissa Baese-Berk \& Matthew Goldrick. 2016. Dimensions of similarity in the mental lexicon. Language, Cognition, and Neuroscience.

Fujimura, O., M. J. Macchi \& L. A. Streeter. 1978. Perception of stop consonants with conflicting transitional cues: A cross-linguistic study. Language and Speech 21.337-46.

Gahl, Susanne \& Susan M. Garnsey. 2004. Knowledge of grammar, knowledge of usage: Syntactic probabilities affect pronunciation variation. Language 80.748-75.

Gahl, Susanne, Susan M. Garnsey, Cynthia Fisher \& Laura Matzen. 2006. "That sound unlikely": Syntactic probabilities affect pronunciation. Proceedings of the 28th Annual Conference of the Cognitive Science Society.1334-39.

Ganong, William F. 1980. Phonetic categorization in auditory word perception. Journal of Experimental Psychology: Human Perception and Performance 6.110-25.

Genzel, Dmitriy \& Eugene Charniak. 2002. Entropy rate constancy in text. Proceedings of the 40th Annual Meeting of the Association for Computational Linguistics.199-206.

Gibson, E., Leon Bergen \& Steven T Piantadosi. 2013a. The rational integration of noisy evidence and prior semantic expectations in sentence interpretation. Proceedings of the National Academy of Sciences 110.8051-56.

Gibson, E., Steven T Piantadosi, K. Brink, Leon Bergen, E. Lim \& R. Saxe. 2013b. A noisychannel account of cross-linguistic word-order variation. Psychological Science 24.1079-88.

Gick, Bryan, Douglas Pulleyblank, Fiona Campbell \& Ngessimo Mutaka. 2006. Low vowels and ATR harmony in Kinande. Phonology 23.1-20.

Gildea, Daniel \& T. Florian Jaeger. Submitted. Human languages order information efficiently.

Goldinger, Stephen D. 2000. The role of perceptual episodes in lexical processing. Proceedings of SWAP (Spoken Word Access Processes), ed. by A. Cutler, J. McQueen \& R. Zondervan, 155-58. Nijmegen: Max Planck Institute for Psycholinguistics.

Goldrick, Matthew, Jocelyn R. Folk \& Brenda Rapp. 2010. Mrs. Malaprop's Neighborhood: Using word errors to reveal neighborhood structure. Journal of Memory and Language 62.113-34.

Goldsmith, John \& Jason Riggle. 2012. Information theoretic approaches to phonology: The case of Finnish vowel harmony. Natural Language and Linguistic Theory 30.859-96.

Graff, Peter. 2012. Communicative efficiency in the lexicon: Massachusetts Institute of Technology Doctoral dissertation. 
Graff, Peter \& T. Florian Jaeger. To appear. Locality and feature-specificity in the OCP: Evidence from Aymara, Dutch, and Javanese. Proceedings from the Annual Meeting of the Chicago Linguistic Society.

Griffin, Zenzi M. \& Victor S. Ferreira. 2006. Properties of spoken language production. Handbook of Psycholinguistics, ed. by M.J. Traxler \& M.A. Gernsbacher, 21-59. Amsterdam: Academic Press.

Grosjean, François. 1980. Spoken word recognition processes and the gating paradigm. Perception and Psychophysics 28.267-83.

Guy, Gregory. 1996. Form and function in linguistic variation. Towards a social science of language: Papers in honor of William Labov, ed. by G. Guy, C. Feagin, D. Schiffrin \& J. Baugh, 221-52. Amsterdam: John Benjamins.

Hall, Daniel Currie. 2011a. Phonological contrast and its phonetic enhancement: Dispersedness without dispersion. Phonology 28.1-54.

Hall, Kathleen Currie. 2009. A probabilistic model of phonological relationships from contrast to allophony. Columbus, OH: The Ohio State University Doctoral dissertation.

- 2012. Phonological relationships: A probabilistic model. McGill Working Papers in Linguistics 22.

- 2013a. Documenting phonological change: A comparison of two Japanese phonemic splits. Proceedings of the 2013 Annual Meeting of the Canadian Linguistic Association, ed. by S. Luo. Toronto: Canadian Linguistic Association.

Hall, Kathleen Currie \& Elizabeth Hume. 2015. Modeling perceived similarity: The influence of phonetics, phonology, and frequency on the perception of French vowels (ed.) University of British Columbia, University of Canterbury.

Hall, Kathleen Currie, Hanna Smith, Kevin McMullin, Blake Allen, Noriko Yamane \& Joash Gambarage. 2014. Articulatory correlates of phonological relationships. Paper presented to the 14th Meeting of the Association for Laboratory Phonology, Tokyo, 2014.

Hall, Nancy. 2011b. Vowel epenthesis. The Blackwell Companion to Phonology, ed. by M. van Oostendorp, C.J. Ewen, E. Hume \& K. Rice, 1576-96. Malden, MA: Wiley-Blackwell.

-. 2013b. Acoustic differences between lexical and epenthetic vowels in Lebanese Arabic. Journal of Phonetics 41.133-43.

Hansson, Gunnar Ólafur. 2003. Laryngeal licensing and laryngeal neutralization in Faroese and Icelandic. Nordic Journal of Linguistics 26.45-79.

Harris, John. 2011. Deletion. The Blackwell Companion to Phonology, ed. by M. van Oostendorp, C.J. Ewen, E. Hume \& K. Rice, 1597-621. Malden, MA: Wiley-Blackwell.

Haspelmath, Martin. 2006. Against markedness (and what to replace it with). Journal of Linguistics 42.25-70.

Hawkins, Sarah. 2003. Roles and representations of systematic fine phonetic detail in speech understanding. Journal of Phonetics 31.373-405.

Hay, Jennifer B., Janet B. Pierrehumbert, Abby J. Walker \& Patrick LaShell. 2015. Tracking word frequency effects through 130 years of sound change. Cognition 139.83-91.

Hay, Jennifer \& Margaret MacLagan. 2012. /r/-sandhi in early 20th-century New Zealand English. Linguistics 50.745-63.

Hayes, Bruce. 1999. Phonetically driven phonology: The role of optimality in inductive grounding. Functionalism and formalism in linguistics, ed. by M. Darnell, E. Moravcsik, M. Noonan, F. Newmeyer \& K. Wheatly, 243-85. Amsterdam: John Benjamins. 
Hayes, Bruce, Robert Kirchner \& Donca Steriade. 2004. Phonetically Based Phonology Cambridge: Cambridge University Press.

Hockett, Charles. 1955. Manual of Phonology. International Journal of American Linguistics 21.

Hollenbach, Barbara E. 1984. The phonology and morphology of tone and laryngeals in Copala Trique: University of Arizona Doctoral dissertation.

Hong, Sung-Hoon. 2011. Information-theoretic account of vowel epenthesis in Korean. Paper presented at the Information-Theoretic Approaches to Linguistics, University of Colorado, Boulder.

Hume, Elizabeth. 1998. The role of perceptibility in consonant/consonant metathesis. Proceedings of West Coast Conference on Formal Linguistics 17.293-307.

- 2003. Language specific markedness: The case of place of articulation. Studies in Phonetics, Phonology, and Morphology 9.295-310.

- 2004. The indeterminacy / attestation model of metathesis. Language 80.203-37.

-. 2005. Deconstructing markedness: A predictability-based approach. Proceedings of the 13th Annual Meeting of the Berkeley Linguistics Society, ed. by M. Ettlinger, N. Fleisher \& M. Park-Doob, 182-98. Berkeley, CA: Berkeley Linguistics Society.

- 2016. Phonological markedness and its relation to the uncertainty of words. Phonological Studies: Journal of the Phonological Society of Japan 19.

Hume, Elizabeth \& Ilana Bromberg. 2005. Predicting epenthesis: an information-theoretic account. Paper presented to the 7th Annual Meeting of the French Network of Phonology, Aix-en-Provence, 2005.

Hume, Elizabeth, Kathleen Currie Hall \& Andrew Wedel. 2016. Perceptually strong and weak unmarked patterns: A message-based approach. Proceedings of the Annual Meeting of Phonology.

Hume, Elizabeth, Kathleen Currie Hall, Andrew Wedel, Adam Ussishkin, Martine Adda-Decker \& Cédric Gendrot. 2013. Anti-markedness patterns in French epenthesis: An informationtheoretic approach. Proceedings of the Thirty-Seventh Annual Meeting of the Berkeley Linguistics Society, ed. by C. Cathcart, I.-H. Chen, G. Finley, S. Kang, C.S. Sandy \& E. Stickles, 104-23. Berkeley: Berkeley Linguistics Society.

Hume, Elizabeth \& Keith Johnson. 2001. A model of the interplay of speech perception and phonology. The role of speech perception in phonology, ed. by E. Hume \& K. Johnson, 3-26. San Diego: Academic Press.

Hume, Elizabeth \& Frédéric Mailhot. 2013. The role of entropy and surprisal in phonologization and language change. Origins of sound patterns: Approaches to phonologization, ed. by A. Yu, 29-50. Oxford: Oxford University Press.

Hume, Elizabeth, Jennifer Muller \& Aone van Engelenhoven. 1997. Non-moraic geminates in Leti. Phonology 14.371-402.

Hume, Elizabeth, Darcy Rose \& Michael Spagnol. 2014. Maltese word-initial singleton-geminate contrasts: An informatioin-theoretic approach. Where the Principles Fail, ed. by R. Kager, J. Grijzenhout \& K. Sebregts, 89-101. Utrecht: OTS.

Hume, Elizabeth \& Giorgios Tserdanelis. 2002. Labial unmarkedness in Sri Lankan Portuguese Creole. Phonology 19.441-58.

Itô, Junko. 1986. Syllable theory in prosodic phonology New York: Garland.

-. 1989. A prosodic theory of epenthesis. Natural Language and Linguistic Theory 7.217-60.

Jaeger, T. Florian. 2006. Redundancy and syntactic reduction in spontaneous speech: Stanford University Doctoral dissertation. 
- 2010. Redundancy and reduction: Speakers manage syntactic information density. Cognitive Psychology 61.23-62.

- 2011. Corpus-based research on language production: Information density and reducible subject relatives. Language from a cognitive perspective: Grammar, usage, and processing, ed. by E.M. Bender \& J.E. Arnold, 161-97. Stanford: CSLI Publications.

- 2013. Production preferences cannot be understood without reference to communication. Frontiers in Psychology 4.

Jaeger, T. Florian \& Esteban Buz. To appear. Signal reduction and linguistic encoding. Handbook of psycholinguistics, ed. by E.M. Fernandez \& H.S. Cairns. Oxford: WileyBlackwell.

Jaeger, T. Florian \& Victor S. Ferreira. 2013. Seeking predictions from a predictive framework. Behavioral and brain sciences 36.31-32.

Jaeger, T. Florian \& Harry Tily. 2011. Language processing complexity and communicative efficiency. WIRE: Cognitive Science 2. 323-35.

Johnson, Keith. 1997. The auditory/perceptual basis for speech segmentation. Papers from the linguistics laboratory, ed. by K. Ainsworth-Darnell \& M. D'Imperio, 101-13. Columbus, OH: Ohio State University Working Papers in Linguistics.

Johnson, Keith. 2006. Resonance in an exemplar-based lexicon: The emergence of social identity and phonology. Journal of Phonetics 34.485-99.

Ju, Min \& Paul A. Luce. 2006. Representational specificity of within-category phonetic variation in the long-term mental lexicon. Journal of Experimental Psychology: Human Perception and Performance 32.120-38.

Jun, Jongho. 1995. Perceptual and articulatory factors in place assimilation: An Optimality Theoretic approach: UCLA Doctoral dissertation.

—. 2004. Place assimilation. Phonetically based phonology, ed. by B. Hayes, R. Kirchner \& D. Steriade, 58-86. Cambridge: Cambridge University Press.

-. 2011. Positional effects in consonant clusters. The Blackwell Companion to Phonology, ed. by M. van Oostendorp, C.J. Ewen, E. Hume \& K. Rice, 1103-23. Malden, MA: WileyBlackwell.

Jurafsky, Dan. 2003. Probabilistic modeling in psycholinguistics: Linguistic comprehension and production. Probabilistic Linguistics, ed. by R. Bod, J. Hay \& S. Jannedy, 39-96. Cambridge, MA: MIT Press.

Jurafsky, Daniel. 1996. A Probabilistic Model of Lexical and Syntactic Access and Disambiguation. Cognitive Science 20.137-94.

Jurafsky, Daniel, Alan Bell, Michelle Gregory \& William Raymond. 2001. Probabilistic relations between words: evidence from reduction in lexical production. Frequency and the emergence of linguistic structure, ed. by J.L. Bybee \& P. Hopper, 229-54. Amsterdam: Benjamins.

Jurafsky, Daniel \& James H. Martin. 2000. Speech and Language Processing: An Introduction to Natural Language Processing, Computational Linguistics, and Speech Recognition Upper Saddle River, NJ: Prentice Hall.

Kawahara, Shigeto \& Kelly Garvey. 2014. Nasal place assimilation and the perceptibility of place contrasts. Open Linguistics 1.17-36.

Kehler, Andrew \& Hannah Rohde. 2013. A probabilistic reconciliation of coherence-driven and centering-driven theories of pronoun interpretation. Theoretical Linguistics 39.1-37.

Kei, Joseph, Veronica Smyth, Lydia K. H. So, C. C. Lau \& K. Capell. 2002. Assessing the accuracy of production of Cantonese lexical tones: A comparison between perceptual 
judgment and an instrumental measure. Asia Pacific Journal of Speech, Language, and Hearing 7.25-38.

Kewley-Port, Diane \& Malcolm S. Preston. 1974. Early apical stop production: A voice onset time analysis. Journal of Phonetics 2.195-210.

Keyser, Samuel Jay \& Wayne A. O'Neil. 1985. Rule generalization and optionality in language change Dordrecht: Foris Publications.

Kim, Kyumin \& Alexei Kochetov. 2011. Phonology and phonetics of epenthetic vowels in loanwords: Experimental evidence from Korean. Lingua 121.511-32.

King, Jeannete, Margaret Maclagan, Ray Harlow, Peter J. Keegan \& Catherine Watson. 2010. The MAONZE corpus: Establishing a corpus of Maori speech. New Zealand Studies in Applied Linguistics 16.1-16.

Kingston, John. 2007. The phonetics-phonology interface. The handbook of phonology, ed. by P. de Lacy, 401-34. Cambridge: Cambridge University Press.

Kiparsky, Paul. 1982. Lexical morphology and phonology. Linguistics in the Morning Calm, ed. by T.L.S.o. Korea, 3-91. Seoul: Hanshin.

Kirby, James. 2010. Cue selection and category restructuring in sound change: University of Chicago Doctoral dissertation.

Kirby, Simon, Monica Tamariz, Hannah Cornish \& Kenny Smith. 2015. Compression and communication in the cultural evolution of linguistic structure. Cognition 141.87-102.

Kirchner, Robert. 2001. An effort-based approach to consonant lenition. New York, NY: Routledge.

Kirov, Christo \& Colin Wilson. 2012. The specificity of online variation in speech production. Proceedings of the 34th Annual Conference of the Cognitive Science Society.587-92.

- 2013. Bayesian speech production: Evidence from latency and hyperarticulation. Proceedings of the 35th Annual Meeting of the Cognitive Science Society.788-93.

Kisseberth, Charles W. 1970. On the functional unity of phonological rules. Linguistic Inquiry 1.291-306.

Kleinschmidt, Dave F. \& T. Florian Jaeger. 2015. Robust speech perception: Recognizing the familiar, generalizing to the similar, and adapting to the novel. Psychological Review 122.148-203.

-. 2016. Re-examining selective adapatation: Fatiguing feature detectors, or distributional learning? Psychonomic Bulletin and Review 23.678-91.

Kochetov, Alexei. 2002. Production, Perception and Emergent Phonotactic Patterns. New York, London: Routledge.

Kohler, Klaus. 1990. Segmental reduction in connected speech in German: Phonological factors and phonetic explanations. Speech production and speech modeling, ed. by W.J. Hardcastle \& A. Marchal, 69-92. Dordrecht: Kluwer.

Kraljic, Tanya \& Arthur G. Samuel. 2005a. Perceptual learning for speech: is there a return to normal? Cognitive Psychology 51.141-78.

-. 2005b. Prosodic disambiguation of syntactic structure: For the speaker or for the addressee? Cognitive Psychology 50.194-231.

Krämer, Martin. 2006. The emergence of the comparatively unmarked. Proceedings of the 25th West Coast Conference on Formal Linguistics, ed. by D. Baumer, D. Montero \& M. Scanlon. Somerville, MA: Cascadilla Proceedings Project. 
Kravtchenko, Ekaterina. 2014. Predictability and syntactic production: Evidence from subject omission in Russian. Proceedings of the 36th Annual Cognitive Science Society Conference.785-590.

Kruschke, John K. 2015. Doing Bayesian data analysis: A tutorial with R, JAGS, and Stan London: Elsevier.

Kuijpers, Cecile, Wilma van Donselaar \& Anne Cutler. 1996. Phonological variation: Epenthesis and deletion of schwa in Dutch. Proceedings of the Fourth International Conference on Spoken Language 1.149-52.

Kuperberg, Gina R. \& T. Florian Jaeger. 2016. What do we mean by prediction in language comprehension? Language Cognition and Neuroscience 31.32-59.

Kuperman, Victor \& Joan Bresnan. 2012. The effects of construction probability on word durations during spontaneous incremental sentence production. Journal of Memory and Language 66.588-611.

Kuperman, Victor, M. Pluymaekers, Mirjam Ernestus \& R. H. Baayen. 2007. Morphological predictability and acoustic duration of interfixes in Dutch compounds. Journal of the Acoustical Society of America 121.2261-71.

Kurumada, Chigusa. 2011. Syntactic context affects probability estimation: Evidence from Japanese relative clauses (ed.) S. University.

Kurumada, Chigusa \& T. Florian Jaeger. 2015. Communicative efficiency in lannguage production: Optional case-marking in Japanese. Journal of Memory and Language 83.152-78.

Labov, William. 1994. Principles of linguistic change: Internal factors Oxford, UK ; Cambridge, MA: Blackwell.

Lahiri, Aditi \& Henning Reetz. 2002. Underspecified recognition. Proceedings of Laboratory Phonology 7, ed. by C. Gussenhoven, N. Warner \& T. Rietveld, 637-76. Berlin: Mouton de Gruyter.

Laplace, Pierre Simon. 1812. Théorie analytique des probabilités Paris: Libraire pour les mathématiques et et la marine.

Lavoie, Lisa. 2002. Some influences on the realization of 'for' and 'four' in American English. Journal of the International Phonetic Association 32.175-202.

Levelt, Clara C., Niels O. Schiller \& Willem J. M. Levelt. 1999. A developmental grammar for syllable structure in the production of child language. Brain and Language 68.291-99.

Levy, Roger \& T. Florian Jaeger. 2007. Speakers optimize information density through syntactic reduction.

Lindblom, Björn. 1990. Explaining phonetic variation: A sketch of the H\&H theory. Speech production and speech modelling, ed. by W.J. Hardcastle \& A. Marchal, 403-39. Dordrecht: Kluwer.

Lozano, María del Carmen. 1979. Stop and spirant alternations: Fortition and spirantization processes in Spanish phonology. Bloomington, IN, Indiana University Linguistics Club: The Ohio State University Doctoral dissertation.

Luce, Paul A. \& David B. Pisoni. 1998. Recognizing spoken words: The neighborhood activation model. Ear Hear 19.1-36.

Lynch, Scott M. 2007. Introduction to applied Bayesian statistics and estimation for social scientists New York: Springer.

MacKay, David J. C. 2003. Information theory, inference, and learning algorithms Cambridge: Cambridge University Press. 
Magnuson, James S., J. A. Dixon, Michael K. Tanenhaus \& Richard N. Aslin. 2007. The dynamics of lexical competition during spoken word recognition. Cognitive Science 31.13356.

Mahowald, Kyle, Evelina Fedorenko, Steven T Piantadosi \& Edward Gibson. 2013. Info/information theory: Speakers choose shorter words in predictive contexts. Cognition 126.313-18.

Malécot, André. 1956. Acoustic cues for nasal consonants: an experimental study using tapesplicing technique. Language 32.274-84.

Malone, Joseph L. 1993. Tiberian Hebrew Phonology Winona Lake: Eisenbrauns.

Manin, Dmitri. 2006. Experiments on predictability of word in context and information rate in natural language. Journal of Information Processes 6.229-36.

Manning, Christopher \& Hinrich Schütze. 1999. Foundations of Statistical Natural Language Processing Cambridge, MA: Massachusetts Institute of Technology.

Martin, Alexander \& Sharon Peperkamp. 2016. Coalescing sources of bias in perception: Lexical and prelexical influences on the processing of phonological features. Paper presented at the 15th Conference on Laboratory Phonology, Cornell University.

Martinet, André. 1955. Économie des changements phonétiques Berne: Francke.

-. 1972. Function, structure, and sound change. Reader in Historical and Comparative Linguistics, ed. by A.R. Keiler, 139-74. New York: Holt, Reinhart, \& Winston, Inc.

Maurits, Luke, Amy Perfors \& Daniel Navarro. 2010. Why are some word orders more common than others? A uniform information density account. Proceedings of the Neural Information Processing Systems conference.

McCarthy, John J. \& Alan Prince. 1995. Faithfulness and reduplicative identity. Papers in Optimality Theory, ed. by J. Beckman, L.W. Dickey \& S. Urbanczyk, 249-384. Amherst, MA: Graduate Linguistic Student Association.

McClelland, J. L., Mark St. John \& Roman Taraban. 1989. Sentence comprehension: A parallel distributed processing approach. Language and Cognitive Processes 4.287-335.

Mesgarani, Nima, Connie Cheung, Keith Johnson \& Edward F. Chang. 2014. Phonetic feature encoding in human temporal gyrus. Science 343.1006-10.

Mielke, Jeff. 2008. The emergence of distinctive features Oxford: Oxford University Press.

Mielke, Jeff \& Elizabeth Hume. 2001. Consideratioins of word recognition for metathesis. Surface syllable structure and segment sequencing, ed. by E. Hume, N. Smith \& J. van de Weijer, 135-58. Leiden: Holland Institute of Generative Linguistics.

Mitterer, Holger \& James McQueen. 2009. Processing reduced word-forms in speech perception using probabilistic knowledge about speech production. Journal of Experimental Psychology: Human Perception and Performance 35.244-63.

Mohanan, K. P. 1993. Fields of attraction in phonology. The Last Phonological Rule: Reflections on Constraints and Derivations, ed. by J. Goldsmith, 61-116. Chicago: University of Chicago Press.

Mok, Peggy \& Peggy Wong. 2010a. Production and perception of the rising tones in Hong Kong Cantonese. Proceedings of the 9th Phonetics Conference of China. Tianjin.

- 2010b. Production of the merging tones in Hong Kong Cantonese: Preliminary data on monosyllables. Proceedings of Speech Prosody 2010, 1-4. Chicago.

Munson, Benjamin \& Nancy P. Solomon. 2004. The effect of phonological neighborhood density on vowel articulation. Journal of Speech, Language, and Hearing Research 47.1048-58.

Nettle, Daniel. 1999. Is the rate of linguistic change constant? Lingua 108.119-36. 
Neumann, Rebecca , Anna N. Rafferty \& Thomas L. Griffiths. 2014. A bounded rationality account of wishful thinking. Proceedings from the 36th Annual Conference of the Cognitive Science Society.

Ní Chiosáin, Máire \& Jaye Padgett. 2001. Markedness, segment realization, and locality in spreading. Segmental phonology in Optimality Theory: Constraints and representations, ed. by L. Lombardi, 118-56. Cambridge: Cambridge University Press.

Nielsen, Kuniko. 2007. Implicit phonetic imitation is constrained by phonemic contrast. Proceedings of the 16th International Congress of Phonetic Sciences.1961-64.

Nielsen, Kuniko \& Colin Wilson. 2008. A hierarchical Bayesian model of multi-level phonetic imitation. Proceedings of the 27th West Coast Conference on Formal Linguistics, ed. by N. Abner \& J. Bishop, 335-43. Somerville, MA: Cascadilla Press.

Norris, Dennis \& James M. McQueen. 2008. Shortlist B: A Bayesian model of continuous speech recognition. Psychological Review 115.357-95.

Norris, Dennis, James M. McQueen \& Anne Cutler. 2003. Perceptual learning in speech. Cognitive Psychology 47.204-38.

Nowak, Martin A. \& David C. Krakauer. 1999. The evolution of language. Proceedings of the National Academy of Sciences 96.8028-33.

O'Donnell, Timothy J. 2015. Productivity and reuse in language: A theory of linguistic computation and storage Cambridge, MA: MIT Press.

Obleser, Jonas, Richard J. S. Wise, M. Alex Dresner \& Sophie K. Scott. 2007. Functional integration across brain regions improves speech perception under adverse listening conditions. The Journal of Neuroscience 27.2283-89.

Oh, Y., C. Coupé, E. Marsico \& F. Pellegrino. 2015. Bridging phonological system and lexicon: Insights from a corpus study of functional load. Journal of Phonetics 53.153-76.

Ohala, John J. 1981. The listener as a source of sound change. Papers from the parasession on language behavior, ed. by C.S. Masek, R.A. Hendrick \& M.F. Miller, 178-203. Chicago: Chicago Linguistic Society.

-. 1988. Discussion of Lindblom's 'Phonetic invariance and the adaptive nature of speech'. Working models of human perception, ed. by B.A.G. Elsendoorn \& H. Bouma, 175-83. London: Academic Press.

- 1989. Sound change is drawn from a pool of synchronic variation. Language change: Contributions to the study of its causes, ed. by L.E. Breivik \& E.H. Jahr, 173-98. Berlin: Mouton de Gruyter.

Ohala, John J. \& Manjari Ohala. 1993. The phonetics of nasal phonology: Theorems and data. Nasals, nasalization, and the velum, ed. by M. Huffman \& R. Krakow, 225-49. New York: Academic Press.

Otake, Takashi, Giyoo Hatano \& Kiyoko Yoneyama. 1996a. Speech segmentation by Japanese listeners. Phonological structure and language processing: Cross-linguistic studies, ed. by T. Otake \& A. Cutler, 183-202. Berlin: Mouton de Gruyter.

Otake, Takashi, Kiyoko Yoneyama, Anne Cutler \& Arie van der Lugt. 1996b. The representation of Japanese moraic nasals. Journal of the Acoustical Society of America 100.3831-42.

Padgett, Jaye. 2009. Systemic contrast and Catalan rhotics. The Linguistic Review 26.431-63.

Paradis, Carole \& Darlene LaCharité. 1997. Preservation and minimality in loanword adaptation. Journal of Linguistics 33.379-430. 
Paradis, Carole \& Jean-François Prunet. 1991a. Introduction: Asymmetry and visibility in consonant articulations. The special status of coronals: Internal and external evidence, ed. by C. Paradis \& J.-F. Prunet, 1-28. San Diego: Academic Press.

- (eds) 1991b. The Special Status of Coronals: Internal and External Evidence (Phonetics and Phonology. San Diego: Academic Press.

Pardo, Jennifer S. 2006. On phonetic convergence during conversational interaction. Journal of the Acoustical Society of America 119.2382-93.

Parlato-Oliveira, Erika, Anne Christophe, Yuki Hirose \& Emmanuel Dupoux. 2010. Plasticity of illusory vowel perception in Brazilian-Japanese bilinguals. Journal of the Acoustical Society of America 127.3738-48.

Pasley, Brian N., Stephen V. David, Nima Mesgarani, Adeen Flinker, Shihab A. Shamma, Nathan E. Crone, Robert T. Knight \& Edward F. Chang. 2012. Reconstructing speech from human auditory cortex. PLoS Biol 10.

Pate, John K \& Sharon Goldwater. 2015. Talkers account for listener and channel characteristics to communicate efficiently. Journal of Memory and Language 78.1-17.

Pellegrino, F., C. Coupé \& E. Marsico. 2011. A cross-language perspective on speech information rate. Language 87.539-58.

Peramunage, D., S. E. Blumstein, E. B. Myers, M. Goldrick \& M. Baese-Berk. 2011. Phonological neighborhood effects in spoken word production: An fMRI study. Journal of Cognitive Neuroscience 23.593-603.

Phillips, Betty. 1984. Word frequency and the actuation of sound change. Language 60.320-42.

—. 2006. Word frequency and lexical diffusion Basingstoke: Palgrave Macmillan.

Piantadosi, Steven T, Harry Tily \& Edward Gibson. 2011. Word lengths are optimized for efficient communication. Proceedings of the National Academy of Sciences 108.3526-29.

- 2012. The communicative function of ambiguity in language. Cognition 122.280-91.

Pierce, John R. 1961. An introduction to Information theory: symbols, signals, and noise New York, NY: Dover Publications.

Pierrehumbert, Janet B. 2001. Exemplar dynamics: Word frequency, lenition, and contrast. Frequency effects and the emergence of lexical structure, ed. by J.L. Bybee \& P. Hopper, 137-57. Amsterdam: John Benjamins.

- 2002. Word-specific phonetics. Laboratory Phonology VII, ed. by C. Gussenhoven \& N. Warner, 101-39. Berlin: Mouton de Gruyter.

-. 2003a. Phonetic diversity, statistical learning, and acquisition of phonology. Language and Speech 46.115-54.

- 2003b. Probabilistic phonology: discrimination and robustness. Probabilistic linguistics, ed. by R. Bod, J. Hay \& S. Jannedy, 177-228. Cambridge, Mass.: MIT Press.

Pisoni, David B. \& Susannah V. Levi. 2007. Some observations on representations and representational specificity in speech perception and spoken word recognition. The Oxford Handbook of Psycholinguistics, ed. by M.G. Gaskell. Oxford: Oxford University Press.

Pitt, Mark A., Laura Dilley, Keith Johnson, Scott Kiesling, William Raymond, Elizabeth Hume \& Eric Fosler-Lussier. 2007. Buckeye Corpus of Conversational Speech (2nd Release). Columbus, OH: Department of Psychology, Ohio State University.

Plotkin, Joshua B. \& Martin A. Nowak. 2000. Language evolution and information theory. Journal of Theoretical Biology 205.147-59.

Pluymaekers, M., Mirjam Ernestus \& R. H. Baayen. 2005a. Articulatory planning is continuous and sensitive to informational redundancy. Phonetica 62.146-59. 
-. 2005b. Lexical frequency and acoustic reduction in spoken Dutch. Journal of the Acoustical Society of America 118.2561-69.

Potts, Christopher, Daniel Lassiter, Roger Levy \& Michael C. Frank. In press. Embedded implicatures as pragmatic inferences under compositional lexical uncertainty. Journal of Semantics.

Pouplier, Marianne. 2012. The gaits of speech: re-examining the role of articulatory effort in spoken language. In Maria-Josep Solé, Daniel Recasens (eds). The Initiation of Sound Change: Perception, Production, and Social Factors. Amsterdam: John Benjamins. 147-166.

Prince, Alan \& Paul Smolensky. 2008. Optimality Theory: Constraint interaction in Generative Grammar Malden, MA: Wiley-Blackwell.

Purnell, Thomas, Dilara Tepeli \& Joseph Salmons. 2005. German substrate effects in Wisconsin English: Evidence for final fortition. American Speech 80.135-64.

Raymond, William, Robin Dautricourt \& Elizabeth Hume. 2006. Word-internal t/d deletion in spontaneous speech: The effects of lexical, phonological, and extra-linguistic factors. Language Variation and Change 18.55-97.

Repp, Bruno H. 1982. Phonetic trading relations and context effects: New experimental evidence for a speech mode of perception. Psychological Bulletin 92.81-110.

Resnik, Philip. 1996. Selectional constraints: An information-theoretic model and its computational realization. Cognition 61.127-59.

Rice, Keren. 1999/2000. Featural markedness in phonology: Variation. GLOT International 4.7, 4.8.3-6, 3-7.

Rice, Keren \& Peter Avery. 1991. On the relationship between laterality and coronality. The special status of coronals: Internal and external evidence, ed. by C. Paradis \& J.-F. Prunet, 101-24. San Diego: Academic Press.

Rohde, Hannah, Roger Levy \& Andrew Kehler. 2011. Anticipating explanations in relative clause processing. Cognition 118.339-58.

Rose, Darcy, Jennifer Hay \& Elizabeth Hume. in prep. Influence of predictability and phonological structure on English plural /s/ duration.

Sadat, Jasmin, Clara D. Martin, Albert Costa \& F.-Xavier Alario. 2014. Reconciling phonological neighborhood effects in speech production through single trial analysis. Cognitive Psychology 68C.33-58.

Saffran, Jenny R., Elissa L. Newport \& Richard N. Aslin. 1996. Word segmentation: The role of distributional cues. Journal of Memory and Language 35.606-21.

Samuel, Arthur G. 1981. Phonemic restoration: Insights from a new methodology. Journal of Experimental Psychology: General 110.474-94.

Scarborough, Rebecca A. 2004. Degree of coarticulation and lexical confusabiltiy. Proceedings of the 29th Meeting of the Berkeley Linguistics Society, ed. by P.M. Nowak, C. Yoquelet \& D. Moretensen, 367-78. Berkeley, CA: Berkeley Linguistics Society.

-. 2012. Lexical similarity and speech production: neighborhoods for nonwords. Lingua 112.164-76.

-. 2013. Neighborhood-conditioned patterns in phonetic detail: relating coarticulation and hyperarticulation. Journal of Phonetics 41.491-508.

Schertz, Jessamyn. 2013. Exaggerating featural contrasts in clarifications of misheard speech in English. Journal of Phonetics 41.249-63.

Schuchardt, Hugo. 1872. The exceptionless operation of the sound laws. On sound laws: Against the neogrammarians, 41-72. 
Schwartz, Myrna F., Gary S. Dell, Nadine Martin, Susanne Gahl \& Paula Sobel. 2006. A caseseries test of the interactive two-step model of lexical access: Evidence from picture naming. Journal of Memory and Language 54.228-64.

Selkirk, Elisabeth O. 1982. The syllable. The structure of phonological representations, ed. by H. Van der Hulst \& N. Smith, 337-83. Dordrecht: Foris.

Seo, Misun. 2003. A segment contact account of the patterning of sonorants in consonant clusters: The Ohio State University Doctoral dissertation.

Seyfarth, Scott. 2014. Word informativity influences acoustic duration: Effects of contextual predictability on lexical representation. Cognition 133.140-55.

Seyfarth, Scott, Esteban Buz \& T. Florian Jaeger. 2016. Dynamic hyperarticulation of coda voicing contrasts. Journal of the Acoustical Society of America 139.EL31-37.

Shadlen, Michael N. \& William T. Newsome. 1994. Noise, neural codes, and cortical organization. Current Opinion in Neurobiology 4.569-79.

Shannon, C. E. 1948. A mathematical theory of communication. The Bell System Technical Journal 27.379-423, 623-56.

Shaw, Jason A., Chong Han \& Yuan Ma. 2014. Surviving truncation: Informativity at the interface of morphology and phonology. Morphology 24.407-32.

Silverman, Daniel. 1997. Tone sandhi in Comaltepec Chinantec. Language 73.473-492

Silverman, Daniel. 2012. Neutralization Cambridge: Cambridge University Press.

Sims, Andrea D. \& Jeff Parker. 2016. How inflection class systems work: On the informativity of implicative structure. Word Structure 9.215-39.

Smith, Jennifer. 2001. Lexical category and phonological contrast. Papers in experimental and theoretical linguistics 6: Workshop on the lexicon in phonetics and phonology, ed. by R. Kirchner, J. Pater \& W. Wikely, 61-72. Edmonton: University of Alberta.

-. 2002. Phonological augmentation in prominent positions. Amherst, MA: University of Massachusetts Doctoral dissertation.

Smith, Jennifer L. 2004. Making constraints positional: Toward a compositional model of CON. Lingua 114.1433-64.

-. 2008. Markedness, faithfulness, positions, and contexts: Lenition and fortition in Optimality Theory. Lenition and Fortition, ed. by J. Brandão de Carvalho, T. Scheer \& P. Ségéral, 51960. Berlin / New York: Mouton de Gruyter.

Snedeker, Jesse \& John Trueswell. 2003. Using prosody to avoid ambiguity: Effects of speaker awareness and referential context. Journal of Memory and Language 48.103-30.

Snoeren, Natalie D., Pierre A. Hallé \& Juan Segui. 2006. A voice for the voiceless: Production and perception of assimilated stops in French. Journal of Phonetics 34.241-68.

Sonderegger, Morgan \& Alan Yu. 2010. A rational account of perceptual compensation for coarticulation. Proceedings of the 32nd Annual Conference of the Cognitive Science Society, ed. by S. Ohlsson \& R. Catrambone, 375-80. Austin, TX: Cognitive Science Society.

Stampe, David. 1979. A Dissertation on Natural Phonology New York: Garland.

Stemberger, Joseph P. 2004. Neighbourhood effects on error rates in speech production. Brain and Language 90.413-22.

Stent, Amanda J., Marie K. Huffman \& Susan E. Brennan. 2008. Adapting speaking after evidence of misrecognition: Local and global hyperarticulation. Speech Communication 50.163-78.

Steriade, Donca. 1987. Redundant values. CLS 23: Parasession on autosegmental and metrical phonology, ed. by A. Bosch, B. Need \& E. Schiller, 339-62. Chicago: CLS. 
- 2001. Directional asymmetries in place assimilation: A perceptual account. The Role of Speech Perception in Phonology, ed. by E. Hume \& K. Johnson, 219-50. New York: Academic Press.

- 2008 [2001]. The phonology of perceptibility effects: The P-map and its consequences for constraint organization. The nature of the word: Studies in honor of Paul Kiparsky, ed. by K. Hanson \& S. Inkelas. Cambridge, MA: MIT Press.

Stevens, Kenneth N. \& Sheila E. Blumstein. 1978. Invariant cues for place of articulation in stop consonants. Journal of the Acoustical Society of America 64.1358-68.

Stevens, Kenneth N. \& Samuel Jay Keyser. 1989. Primary features and their enhancement in consonants. Language 65.81-106.

Sumner, Meghan, Chigusa Kurumada, Roey J. Gafter \& Marisa Casillas. 2013. Phonetic variation and the recognition of words with pronunciation variants. Proceedings of the Annual Meeting of the 35th Annual Conference of the Cognitive Science Society.3486-91.

Sumner, Meghan \& Arthur G. Samuel. 2009. The role of experience in the processing of crossdialectal variation. Journal of Memory and Language 60.487-501.

Szostak, Christine M. \& Mark Pitt. 2013. The prolonged influence of subsequent contaxt on spoken word recognition. Attention, perception, and psychophysics 75.1533-46.

Taler, Vanessa, Geoffrey P. Aaron, Lauren G. Steinmetz \& David B. Pisoni. 2010. Lexical neighborhood density effects on spoken word recognition and production in healthy agin. The Journals of Gerontology Series B: Psychological Sciences and Social Sciences 65B.551-60.

Tily, Harry \& Victor Kuperman. 2012. Rational phonological lengthening in spoken Dutch. Journal of the Acoustical Society of America 132.3935-40.

Todd, Simon. 2012. Functional and length-based Māori vowel contrast. Paper presented at the Annual Meeting of the New Zealand Linguistic Society, Auckland.

Tolhurst, Movshon \& Dean. 1983. The statistical reliability of signals in single neurons in cat and monkey visual cortex. Vision Research 23.775-85.

Torreira, F. \& Mirjam Ernestus. 2009. Probabilistic effects on French [t] duration. Proceedings of the 10th Annual Conference of the International Speech Communication Association.448-51.

Toscano, Joseph C. \& Bob McMurray. 2010. Cue integration with categories: Weighting acoustic cues in speech using unsupervised learning and distributional statistics. Cognitive Science 34.434-64.

Trubetzkoy, Nikolai Sergeevich. 1969[1939]. Principles of phonology Berkeley: University of California Press.

Tsui, Tsz-Him. 2012. Tonal variation in Hong Kong Cantonese: Acoustic distance and functional load. Proceedings from the Annual Meeting of the Chicago Linguistic Society 48.579-88.

- 2013. Contrastiveness and diachronic variation in Chinese nasal codas. Proceedings of the 29th Northwest Linguistics Conference.167-73.

Turk, Alice. 2010. Does prosodic constiuency signal relative predictability? A Smooth Signal Signal Redundancy hypothesis. Laboratory Phonology 1.227-62.

Turnbull, Rory, Scott Seyfarth, Elizabeth Hume \& T. Florian Jaeger. Forthcoming. More than reduction: Nasal assimilation trades off redundancy of both trigger and target.

Tyler, Lorraine K. \& Jeanine Wessels. 1983. Quantifying contextual contributions to wordrecognitioin processes. Perception and Psychophysics 34.409-20.

Uffmann, Christian. 2007. Vowel epenthesis in loanword adaptation Berlin: Walter de Gruyter.

Urbanczyk, Suzanne. 2011. Root-affix asymmetries. Blackwell Companion to Phonology, ed. by M. van Oostendorp, C.J. Ewen, E. Hume \& K. Rice, 2490-515. Oxford: Wiley-Blackwell. 
Ussishkin, Adam \& Andrew Wedel. 2002. Neighborhood density and the root-affix distinction. Proceedings of NELS 32, ed. by M. Hirotani, 539-49.

van Donselaar, Wilma, Cecile Kuijpers \& Anne Cutler. 1999. Facilitatory effects of vowel epenthesis on word processing in Dutch. Journal of Memory and Language 41.59-77.

Van Son, R. J. J. H. \& Louis C. W. Pols. 2003. How efficient is speech? Proceedings of the Institute of Phonetic Sciences 25.171-84.

Van Son, R. J. J. H. \& Jan P. H. Van Santen. 2005. Duration and spectral balance of intervocalic consonants: A case for efficient communication. Speech Communication 47.100-23.

Vigliocco, Gabriella \& Robert J. Hartsuiker. 2002. The interplay of meaning, sound, and syntax in sentence production. Psychological Bulletion 128.442.

Walsh, Michael, Bernd Möbius, Travis Wade \& Hinrich Schütze. 2010. Multilevel exemplar theory. Cognitive Science 34.537-82.

Wang, William S.-Y. 1969. Competing changes as a cause of residue. Language 45.9-25.

Wasow, Thomas, T. Florian Jaeger \& David M. Orr. 2011a. Lexical variation in relativizer frequency. Proceedings of the 2005 DGfS workshop "Expecting the unexpected: Exceptions in grammar", ed. by H. Simon \& H. Wiese, 175-96. Berlin / New York: De Gruyter Mouton.

-. 2011b. Response to Kempson's Comments (Lexical variation in relativizer frequency). Proceedings of the 2005 DGfS workshop "Expecting the unexpected: Exceptions in grammar", ed. by H. Simon \& H. Wiese, 205-11. Berlin / New York: De Gruyter Mouton.

Weatherholtz, Kodi, Dave F. Kleinschmidt, Maryam Seifeldin, Chigusa Kurumada \& T. Florian Jaeger. submitted. Speech perception as probabilistic inference under uncertainty based on social-indexical knowledge.

Wedel, Andrew. 2004. Category competition drives contrast maintenance within an exemplarbased production/perception loop. Proceedings of the Seventh Meeting of the ACL Special Interest Group in Computational Phonology, ed. by J. Goldsmith \& R. Wicentowski, 1-10. Barcelona: Association for Computational Linguistics.

—. 2006. Exemplar models, evolution, and language change. The Linguistic Review 23.247-74.

-. 2007. Feedback and regularity in the lexicon. Phonology 24.147-85.

- 2012. Lexical contrast maintenance and the organization of sublexical contrast systems. Language and Cognition 4.319-55.

Wedel, Andrew, Scott Jackson \& Abby Kaplan. 2013a. Functional load and the lexicon: Evidence that syntactic category and frequency relationships in minimal lemma pairs predict the loss of phoneme contrasts in language change. Language and Speech 56.395-417.

Wedel, Andrew, Abby Kaplan \& Scott Jackson. 2013b. High functional load inhibits phonological contrast loss: A corpus study. Cognition 128.179-86.

Wells, John C. 1982. Accents of English Cambridge: Cambridge University Press.

Whalen, Douglas H. 1992. Perception of overlapping segments: Thoughts on Nearey's model. Journal of Phonetics 20.493-96.

Whalen, Douglas H., Arthur S. Abramson, Leigh Lisker \& Maria Mody. 1993. F0 gives voicing information even with unambiguous voice onset times. Journal of the Acoustical Society of America 93.2152-59.

Wilson, Colin \& Lisa Davidson. 2013. Bayesian analysis of non-native cluster production. Proceedings of the Fortieth Annual Meeting of the North East Linguistic Society, ed. by S. Kan, C. Moore-Cantwell \& R. Staubs, 265-78. Amherst, MA: Graduate Linguistics Student Association, University of Massachusetts. 
Winter, Bodo. 2014. Spoken language achieves robustness and evolvability by exploiting degeneracy and neutrality. BioEssays 36.960-67.

Wright, Richard. 1996. Consonant clusters and cue preservation in Tsou: UCLA Doctoral dissertation.

- 2004. Factors of lexical competition in vowel articulation. Phonetic Interpretation: Papers in Laboratory Phonology VI, ed. by J. Local, R. Ogden \& R. Temple, 75-87. Cambridge: Cambridge University Press.

Yu, Alan. 2011. Mergers and neutralization. The Blackwell Companion to Phonology, ed. by M. van Oostendorp, C.J. Ewen, E. Hume \& K. Rice, 1892-918. Oxford: Wiley-Blackwell.

- (ed.) 2013. Origins of sound change. Oxford: Oxford University Press.

Zipf, George Kingsley. 1932. Selected Studies of the Principle of Relative Frequency in Language Cambridge, MA.

Zoll, Cheryl. 2003. Positional asymmetries and licensing. Optimality Theory in Phonology: A Reader, ed. by J.J. McCarthy, 365-78. Oxford, UK: Blackwell. 